Portland State University

PDXScholar

Spring 1-1-2012

\title{
On the Effect of Heterogeneity on the Dynamics and Performance of Dynamical Networks
}

\author{
Alireza Goudarzi \\ Portland State University
}

Follow this and additional works at: https://pdxscholar.library.pdx.edu/open_access_etds

Part of the OS and Networks Commons, and the Systems Architecture Commons Let us know how access to this document benefits you.

\section{Recommended Citation}

Goudarzi, Alireza, "On the Effect of Heterogeneity on the Dynamics and Performance of Dynamical Networks" (2012). Dissertations and Theses. Paper 369.

https://doi.org/10.15760/etd.369

This Thesis is brought to you for free and open access. It has been accepted for inclusion in Dissertations and Theses by an authorized administrator of PDXScholar. Please contact us if we can make this document more accessible: pdxscholar@pdx.edu. 
On the Effect of Heterogeneity on the Dynamics and Performance of Dynamical

\author{
Networks
}

by

Alireza Goudarzi

A thesis submitted in partial fulfillment of the requirements for the degree of

\author{
Master of Science \\ in \\ Systems Science
}

Thesis Committee:

Christof Teuscher, Chair

George G. Lendaris

Gerardo Lafferriere

Portland State University

(C) 2012 


\begin{abstract}
The high cost of processor fabrication plants and approaching physical limits have started a new wave research in alternative computing paradigms. As an alternative to the top-down manufactured silicon-based computers, research in computing using natural and physical system directly has recently gained a great deal of interest. A branch of this research promotes the idea that any physical system with sufficiently complex dynamics is able to perform computation. The power of networks in representing complex interactions between many parts make them a suitable choice for modeling physical systems. Many studies used networks with a homogeneous structure to describe the computational circuits. However physical systems are inherently heterogeneous. We aim to study the effect of heterogeneity in the dynamics of physical systems that pertains to information processing. Two particularly well-studied network models that represent information processing in a wide range of physical systems are Random Boolean Networks (RBN), that are used to model gene interactions, and Liquid State Machines (LSM), that are used to model brain-like networks. In this thesis, we study the effects of function heterogeneity, in-degree heterogeneity, and interconnect irregularity on the dynamics and the performance of RBN and LSM. First, we introduce the model parameters to characterize the heterogeneity of components in RBN and LSM networks. We then quantify the effects of heterogeneity on the network dynamics. For the three heterogeneity aspects that we studied, we found that the effect of heterogeneity
\end{abstract}


on RBN and LSM are very different. We find that in LSM the in-degree heterogeneity decreases the chaoticity in the network, whereas it increases chaoticity in RBN. For interconnect irregularity, heterogeneity decreases the chaoticity in LSM while its effects on RBN the dynamics depends on the connectivity. For $\langle K\rangle<2$, heterogeneity in the interconnect will increase the chaoticity in the dynamics and for $\langle K\rangle>2$ it decreases the chaoticity. We find that function heterogeneity has virtually no effect on the LSM dynamics. In RBN however, function heterogeneity actually makes the dynamics predictable as a function of connectivity and heterogeneity in the network structure. We hypothesize that node heterogeneity in RBN may help signal processing because of the variety of signal decomposition by different nodes. 


\section{ACKNOWLEDGMENTS}

I am indebted to my advisor Christof Teuscher for his support and guidance without which none of this would have been possible. Many indispensable discussions with Guy Feldman and Rajesh Venkatachalapathy have helped to shape this work. This study was supported in part by the National Science Foundation under grant number 1028120 and Sigma Xi grant-in-aid of research under grant number G20111015158246. 


\section{CONTENTS}

Abstract .............................

Acknowledgments . . . . . . . . . . . . . . . iii

List of Tables . . . . . . . . . . . . . . . . . . . . . . vi

List of Figures . . . . . . . . . . . . . . . . . vii

1 Introduction . . . . . . . . . . . . . . . . . . 1

1.1 Motivation . . . . . . . . . . . . . . . . 1

1.2 Systems View of the Heterogeneity in Random Dynamical Networks 2

1.3 Related Work . . . . . . . . . . . . . . . . . 4

1.3.1 Cellular Automata ................. 4

1.3.2 Random Boolean Networks . . . . . . . . . . . . . 5

1.3.3 Liquid State Machines . . . . . . . . . . . . . . 7

1.3.4 Complexity and Heterogeneity in Networks . . . . . . . . . . 8

1.4 My Contributions . . . . . . . . . . . . . . . . 9

2 Models and Measures . . . . . . . . . . . . . . . . . . 11

2.1 Models . . . . . . . . . . . . . . . . . . . 11

2.1.1 Random Boolean Network (RBN) . . . . . . . . . . . . 11

2.1.2 Liquid State Machine (LSM) . . . . . . . . . . . . . . 14

2.2 Entropy and Heterogeneity . . . . . . . . . . . . . . . . . 17

2.3 In-degree Heterogeneity . . . . . . . . . . . . . . . . . . . . . 21

2.3.1 Entropy and In-degree Distribution . . . . . . . . . . . 23

2.3.2 In-degree Sequence as a Dynamical System . . . . . . . . . . 25

2.3.3 Evolution of In-degree Sequence . . . . . . . . . . . . . 26

2.3.4 Maximum Entropy Distribution . . . . . . . . . . . . . 28

2.3.5 Truncated Exponential Distribution . . . . . . . . . . . . . 30

2.3.6 Discussion . . . . . . . . . . . . . . . 34

2.4 Function Heterogeneity . . . . . . . . . . . . . . . . . 35 
2.5 Interconnect Irregularity . . . . . . . . . . . . . . . . . . . . . . . . 39

2.6 Measures of the Network Dynamics . . . . . . . . . . . . . . . . . 40

2.6.1 Derrida Measure . . . . . . . . . . . . . . . . 40

2.6.2 Damage Spreading . . . . . . . . . . . . . . . 42

2.6.3 Lyapunov Exponent for LSM . . . . . . . . . . . . . . 44

2.6.4 Network Topology Measures . . . . . . . . . . . . . . . . . 45

3 In-degree Heterogeneity . . . . . . . . . . . . . . . . . . . . 47

3.1 Dynamics of Random Boolean Networks . . . . . . . . . . . . . . . 47

3.1.1 Derrida Curve and Maximum Entropy In-Degree Distribution 47

3.1.2 Change in Derrida Curve . . . . . . . . . . . . . . . . 48

3.1.3 Optimal Connectivity in Heterogeneous RBN . . . . . . . . 54

3.1.4 Damage Spreading and Heterogeneity . . . . . . . . . . . . . 55

3.2 Dynamics of Liquid State Machine . . . . . . . . . . . . . . . . 57

3.2.1 Lyapunov Exponent and In-degree Heterogeneity . . . . . . 57

3.3 Conclusion . . . . . . . . . . . . . . . . . . 60

4 Function Heterogeneity . . . . . . . . . . . . . . . . . 62

4.1 Function Heterogeneity in Random Boolean Network . . . . . . . . 62

4.2 Function Heterogeneity in Liquid State Machines . . . . . . . . . . 66

4.2.1 Lyapunov Exponent and Function Heterogeneity . . . . . . . 67

4.3 Conclusions ....................... 70

5 Interconnect Irregularity . . . . . . . . . . . . . . . . 78

5.1 Network Locality . . . . . . . . . . . . . . . . . . . . . 78

5.2 Network Topology . . . . . . . . . . . . . . . . . . 80

5.3 Dynamics of Random Boolean Networks . . . . . . . . . . . . . . 83

5.4 Dynamics of Liquid State Machines . . . . . . . . . . . . . . . . . . 84

5.5 Conclusions ........................ 86

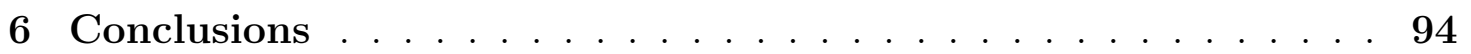

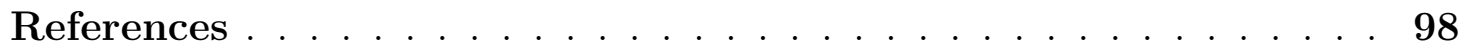




\section{LIST OF TABLES}

6.1 This table summarizes the effects of heterogeneity in different aspects of RBN and LSM on the richness of dynamics. We compared our LSM results to the result for coupled oscillator networks [64]. We also compared our RBN results to scale-free RBNs [5,96]. Our result in the dynamical richness of the network for heterogeneous and homogeneous networks agree with the existing studies on similar networks. . . . . . . . . . . . . . . . . . . . . 97 97 


\section{LIST OF FIGURES}

2.1 Example of RBN with 18 nodes and $\langle K\rangle=2$. Input signals are fed to the network via nodes 1,2 and 3 . One of the nodes (node 18 in this case) is chosen randomly to read the output. . . . . . . . . . 13

2.2 Schematic of a reservoir computing system. The input layer delivers the input signals to random nodes inside the reservoir. The readout layer receives output signals from random nodes inside the reservoir. The reservoir itself is made of a collection of computing nodes that are randomly interconnected. The reservoir creates a representation of the input signals that can be read and classified by the readout layer. Learning is performed by training only the readout layer nodes and connections. . . . . . . . . . . . . .

2.3 The standard deviation $(\sigma)$ and the entropy $H$ of a sample of 100 numbers taken from uniform and normal distributions. We see in the example of the uniform and normal distributions that the same distribution on different values result in different $\sigma$, but the same $\mathcal{H}\left(P_{A}\right)$. However, different distributions might have the same value of $\mathcal{H}\left(P_{A}\right)$, which means we need to control for the distribution in our study. . . . . . . . . . . . . . . . . .

2.4 The parameter space we will use for our study include: the powerlaw exponent of the connection length distribution $\alpha$, entropy of the exponential in-degree distribution, and the standard deviation of the Gaussian distribution for the coefficient of the hyperbolic tangent function $\sigma_{F}$. . . . . . . . . . . . . . . . . . . . . . . 20

2.5 A network with homogeneous or uniform in-degrees, where each node has two inputs $\langle K\rangle=2$. The numbers on the first row are the given node indices. In-degree sequence is the sequence of in-degrees ordered according to the indices of the nodes in the network. Indegree distribution refers to the frequency distribution of the indegrees in the network. . . . . . . . . . . . . . . . . 22 
2.6 A network with heterogeneous (or non-uniform) in-degrees, where each node $i$ has a different number of inputs $K_{i}$. The numbers on the first row are the given node indices. In-degree sequence is the sequence of in-degrees ordered according to the indices of the nodes in the network. In-degree distribution refers to the frequency distribution of the in-degrees in the network. . . . . . . . . . . . 23

2.7 (a) Entropy of in-degree distribution of the network that is undergoing random mutation. The plot corresponds to a single run. We see a rapid increase followed by a fluctuating stationary period, which corresponds to the equilibrium in-degree distribution. In this equilibrium the fluctuations in the entropy is due to random nature of the mutation, but on average the value of the entropy is constant and its trend is stable. The average in-degree $\langle K\rangle=2, N=100$, and average maximum value of the entropy reached is $\mathcal{H}_{\text {max }}=2.7073$. (b) Entropy of in-degree distribution of the network that is undergoing random mutation at low temperature $T=0$. We see a rapid increase followed by a constant entropy period, which corresponds to the equilibrium in-degree distribution. The average in-degree $\langle K\rangle=2, N=100$, and average maximum value of the entropy reached is $\mathcal{H}_{\max }=2.7299$. . . . . . . . . . . . . . . . . . .

2.8 Equilibrium in-degree distribution for maximum entropy networks after evolution. $\langle K\rangle=2$. The resulting distribution looks similar to an exponential distribution. . . . . . . . . . . . . . . . . . . . . 29

2.9 The mean and standard deviation of the coefficient $a$ in the truncated maximum entropy distribution of the form $a e^{-b x}$. . . . . . . 32

2.10 The mean and standard deviation of the coefficient $b$ in the truncated maximum entropy distribution of the form $a e^{-b x}$. . . . . . . 33

2.11 Mean and standard deviation of the entropy of the truncated expo-

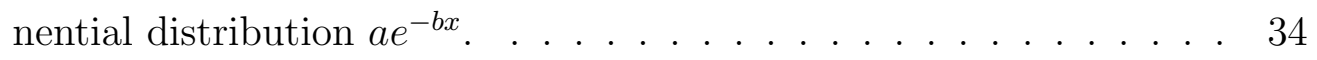

2.12 Hyperbolic tangent for different values of parameter $\alpha . . . .37$

2.13 Curve of the normal distribution for zero mean $(\mu=1)$ and different standard deviation, $\sigma \in\{0.01,0.1,0.4,0.8,10\}$. The equation for the normal distribution is $\mathcal{N}\left(\mu, \sigma^{2}\right)=\frac{1}{\sqrt{2 \pi \sigma^{2}}} e^{\frac{-(x-\mu)^{2}}{2 \sigma^{2}}}$. . . . . . . . . . 
2.14 Power-law distribution for connection length. The nodes are placed on a mesh with a unit distance between two horizontal and vertical neighbors. (a) Probability of a connection between node $\mathrm{A}$ and node $\mathrm{B}$ with distance $r$ is proportional to $r^{-\alpha}$. (b) For $\alpha>0$, the length distribution will be biased toward short range connections. For $\alpha=0$ the link probability is independent of distance between the source and the destination nodes. . . . . . . . . . . . . . .

2.15 Analytical and experimental data for Derrida curve for RBNs with different in-degree distributions. . . . . . . . . . . . . . .

2.16 Derrida curves for simulated systems and the annealed approximation model. The simulation and theoretical prediction for damage spreading in RBN with exactly $\langle K\rangle=2$ connections per node agree closely. For networks with average connectivity $\langle K\rangle=2$ and a Poisson in-degree distribution, the prediction of the annealed model and the simulation diverge. The annealed model predicts a frozen dynamics for Poisson networks while simulations show the system is chaotic. . . . . . . . . . . . . . . . . . 43

3.1 Derrida curve for $\langle K\rangle=1.0,1.5$, and 2.0 for RBN with maximum entropy in-degree distribution. We see that both $\langle K\rangle=1.5$ and 2.0 are in the chaotic regime and $\langle K\rangle=1.0$ is in the critical regime. . . 48

3.2 The Derrida curve of RBN with $N=100$ and $\langle K\rangle=1.0$ for different number of evolutionary time steps. Higher time steps result in more heterogeneous in-degrees. The higher the in-degree heterogeneity, the richer the network dynamics. Curves below the identity line are in ordered regime, and curves above the identity line are in chaotic regime. The closer a curve to the identity line is, the closer the dynamics is to the critical dynamics. . . . . . . . . . . . . 
3.3 The Derrida curve of RBN with $N=100$ and $\langle K\rangle=2.0$ for different number of evolutionary time steps. Higher time steps result in more heterogeneous in-degrees. The higher the in-degree heterogeneity, the richer the network dynamics. In this case, the system is already in critical regime. The higher heterogeneity pushes the system into the chaotic region. Curves below the identity line are in ordered regime, and curves above the identity line are in chaotic regime. The closer a curve to the identity line is, the closer the dynamics is to the critical dynamics. . . . . . . . . . . . . . . . . 51

3.4 The change in $\frac{d_{t+1}}{d_{t}}$ as a function of numbers of time steps. The closer a system is to $\frac{d_{t+1}}{d_{t}}=1$ the richer the dynamics. We observe that high heterogeneity in RBN makes the dynamics of ordinarily ordered systems, i.e., $\langle K\rangle=1.0$, richer while pushing the dynamics of critical systems, i.e., $\langle K\rangle=2.0$, into the chaotic regime. . . . . . 52

3.5 The change in $\frac{d_{t+1}}{d_{t}}$ as a function of entropy of in-degree distribution. The closer a system is to $\frac{d_{t+1}}{d_{t}}=1$ the richer the dynamics. We observe that high heterogeneity in RBN makes the dynamics of ordinarily ordered systems, i.e., $\langle K\rangle=1.0$, richer while pushing the dynamics of critical systems, i.e., $\langle K\rangle=2.0$, into the chaotic regime. 53

3.6 Critical connectivity $\langle K\rangle=K_{c}=1.157$ for which $\frac{d_{t+1}}{d_{t}}=1$ for RBNs with maximum entropy in-degree distribution. For fully heterogeneous RBN the critical connectivity is much lower than that of homogeneous systems, i.e., $K_{c}=2.0$. . . . . . . . . . . . . 55

3.7 Damage spreading in $\mathrm{RBN}$ of $N=100$ and $N=200$. The in-degree distribution is Poissonian. We see that information processing and noise dampening in Poisson networks is at the connectivity $\langle K\rangle \approx$ 1.8. This connectivity balances the communication and resilience of the network. . . . . . . . . . . . . . . 5 56

3.8 Damage spreading in homogeneous networks, Poissonian networks, and exponential networks for $N=100$ and $N=200$. Over long run for static inputs, the effect of heterogeneity in the damage spreading of the network is negligible. . . . . . . . . . . . . 58 
3.9 Damage spreading in networks with heterogeneous in-degree distribution as a function of number mutation in the in-degree distribution $t .\langle K\rangle=1.0$ (blue), $\langle K\rangle=2.0$ (red), $\langle K\rangle=3.0$ (black), $\langle K\rangle=4.0$ (green). High in-degree heterogeneity result in poorer dynamics in LSM. . . . . . . . . . . . . . . . . .

3.10 Lyapunov exponent in networks with heterogeneous in-degree distribution as a function of number mutation in the in-degree distribution $t .\langle K\rangle=1.0$ (blue), $\langle K\rangle=2.0$ (red), $\langle K\rangle=3.0$ (black), $\langle K\rangle=4.0$ (green). High in-degree heterogeneity result in poorer dynamics in LSM. . . . . . . . . . . . . . . . . 61

4.1 NAND function. The decimal value of the function is $0 \times 2^{3}+1 \times$ $2^{2}+1 \times 2^{1}+1 \times 2^{0}=7 \ldots \ldots \ldots . \ldots \ldots 63$

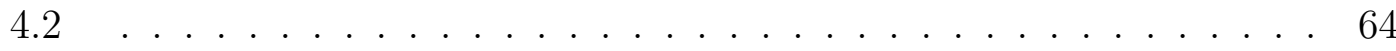

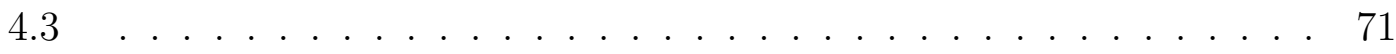

4.4 Damage spreading $H$ in the LSM with binomial in-degree distribution and heterogenous functions with $\langle K\rangle=1$ (blue), $\langle K\rangle=2$ (red), $\langle K\rangle=5$ (black), $\langle K\rangle=10$ (green). Damage spreading in LSM is independent of function heterogeneity in all connectivity levels. . . . 72

4.5 Lyapunov exponent $\lambda$ in the LSM with binomial in-degree distribution and heterogenous functions with $\langle K\rangle=1$ (blue), $\langle K\rangle=2$ (red), $\langle K\rangle=5$ (black), $\langle K\rangle=10$ (green). Dynamics of LSM is independent of function heterogeneity in all connectivity levels. . . . 73

4.6 Damage spreading $H$ in the LSM with exponential in-degree distribution and heterogenous functions with $\langle K\rangle=1$ (blue), $\langle K\rangle=2$ (red), $\langle K\rangle=5$ (black), $\langle K\rangle=10$ (green). Damage spreading in LSM is independent of function heterogeneity in all connectivity

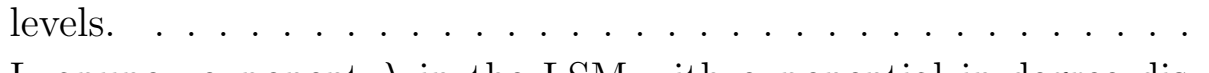

4.7 Lyapunov exponent $\lambda$ in the LSM with exponential in-degree distribution and heterogenous functions with $\langle K\rangle=1$ (blue), $\langle K\rangle=2$ (red), $\langle K\rangle=5$ (black), $\langle K\rangle=10$ (green). Dynamics of LSM is independent of function heterogeneity in all connectivity levels. . . . 75

4.8 Damage spreading $H$ in the LSM with uniform in-degree distribution and heterogenous functions with $\langle K\rangle=1$ (blue), $\langle K\rangle=2$ (red), $\langle K\rangle=5$ (black), $\langle K\rangle=10$ (green). Damage spreading in LSM is independent of function heterogeneity in all connectivity levels. . . . 
4.9 Lyapunov exponent $\lambda$ in the LSM with uniform in-degree distribution and heterogenous functions with $\langle K\rangle=1$ (blue), $\langle K\rangle=2$ (red), $\langle K\rangle=5$ (black), $\langle K\rangle=10$ (green). Dynamics of LSM is independent of function heterogeneity in all connectivity levels. . . . 77

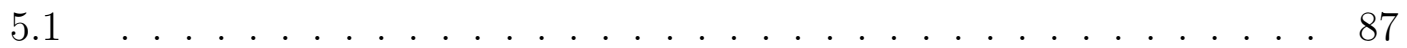

$5.2 \ldots \ldots \ldots \ldots 8 \ldots \ldots \ldots$

5.3 Average local clustering in the networks as a function of $a(\mathrm{a})$ and $\mathcal{H}\left(P_{\mathcal{L}_{a}}\right)(\mathrm{b})$. Blue curve is $K=1$, black curve is $K=2$, magenta curve is $K=3$, and red curve is $K=4$. . . . . . . . . . . . . . . 89

5.4 Average shortest path length $L$ in the networks as a function of $a($ a) and $\mathcal{H}\left(P_{\mathcal{L}_{a}}\right)(\mathrm{b})$. Blue curve is $K=1$, black curve is $K=2$, magenta curve is $K=3$, and red curve is $K=4 \ldots$. . . . . . . . . . . . . 89

5.5 Average modularity $Q$ value in the networks as a function of $a($ a) and $\mathcal{H}\left(P_{\mathcal{L}_{a}}\right)(\mathrm{b})$. Blue curve is $K=1$, black curve is $K=2$, magenta curve is $K=3$, and red curve is $K=4 \ldots$. . . . . . . . . . . 90

5.6 Dynamics of RBN with different connectivity as a function of entropy of wire length distribution. Increasing heterogeneity in the networks pushes the RBN dynamics towards more critical region causing richer dynamics suitable for information processing. . . . . 91

5.7 Damage spreading $H$ as a function of $a(\mathrm{a})$ and $\mathcal{H}\left(P_{\mathcal{L}_{a}}\right)(\mathrm{b})$. Damage spreading does not show any effect due to heterogeneity of the wire lenghts. . . . . . . . . . . . . . . . . . 92

5.8 Lyapunov exponent $\lambda$ as a function of $a(\mathrm{a})$ and $\mathcal{H}\left(P_{\mathcal{L}_{a}}\right)$ (b). Dynamics of LSM become poorer as the heterogeneity of the wire lengths increase. Chaotic systems are pushed towards critical regime while the critical systems are pushed towards ordered regime. . . . . . . . 92 


\section{INTRODUCTION}

\subsection{MOTIVATION}

The increasing cost of processor fabrication plants and approaching the physical limits of silicon-based computers $[17,42]$ have motivated researchers to look for alternative computing paradigms, such as molecular computing, quantum computing, amorphous computing $[1,2,11,16,26,34,86,98,99,101,112,116]$. Some of the recent efforts in natural computing have focused on computing using the intrinsic dynamics of physical systems [95]. One of these paradigms is called Reservoir Computing ( $\mathrm{RC})$.

Researchers have been studying the dynamics of systems and categorized the dynamics into three distinct classes: ordered dynamics, chaotic dynamics, and complex or critical dynamics $[52,56,114]$. Several studies have investigated the relationship between the dynamical phase and the computational power of these simple systems $[24,40,63,71,72,79,81]$.

In the same context, we are interested in computing with existing physical systems and we would like to find out how certain physical properties of these systems affect their intrinsic dynamics. Because of the generality of network models to describe complex physical and real-life systems [13], we model the building blocks and their interactions of generic physical systems using networks and study their 
dynamics. Three relevant aspects of networks are: node in-degrees, node func-

tions, and the connectivity patterns between the nodes. A great deal of research in RC systems has focused on using homogeneous random networks to model cortical microcircuits in the brain [74]. These homogeneous networks have identical nodes, each with the same number of input connections and the same transfer function. However, real physical systems violate these assumptions and much of their complex behavior have been attributed to their non-uniformities [20, 25,65].

\subsection{SYSTEMS VIEW OF THE HETEROGENEITY IN RANDOM DYNAMICAL NETWORKS}

To study the effects of heterogeneity on the network dynamics, we have taken a systems perspective [66] in framing our questions and analyzing our results. The systems approach framework to scientific inquiry relies heavily on the observer or the problem solver and his or her intention. It is the observer who defines the boundaries of a system by defining the collective properties of a set of interacting components that is related to problem to be solved. In this view, the observer would be interested to understand the relationship between the components of the system and their interactions, and how the properties of these interacting components affect the systems behavior that he or she would be interested in. The goal of such a scientific study would be to develop understanding and laws with predictive power that can give us control over the collective properties of the system through possible modifications to the systems components that are relevant within the context of the study.

In this thesis, we have identified the context as the computing with physical systems. The objects of our study are generic physical systems used in molecular and brain-like computing. We have accordingly chosen the network models that include relevant details of the systems that we would like to study. The subject of our study is how the system or global dynamics is influenced by the heterogeneity 
in the subcomponents, such as the node in-degrees, the node functions, and the interconnect irregularity. We would like to find the optimal heterogeneity level in these components that let the system remain in a critical systems dynamics that is optimal for complex computation.

The purpose of this thesis is to investigate the effects of heterogeneity in the dynamics of random dynamical networks. We would like to find out how heterogeneity in any of the three aspects mentioned above may affects the dynamics and if there are optimal levels of heterogeneity to achieve critical dynamics in the system. We choose two well-studied network models with complex dynamics that allow us to investigate all these aspects of interest in dynamical networks: Random Boolean Network (RBN) [52] and Liquid State Machine (LSM) [75]. Both of these network models have been used in the context of reservoir computing $[74,102]$ and much literature exists on their dynamical properties [24,30,35, 51, 69, 70, 89-92, 100, 104].

Research in complex networks has so far focused on the heterogeneity in the in-degrees of the network (see Section 1.3). However in this literature, the heterogeneity refers to how many different classes of in-degrees are present. We argue that the conventional definition of heterogeneity does not capture the diversity of in-degrees in the network (see Chapter 2). Studies about topological evolution of RBN have consistently reported emergence of non-trivial in-degrees and small-world topologies in the networks. However, the relationship between the indegrees, the topology, and the dynamics has not been directly studied with respect to the heterogeneity in the network. We will introduce a more pragmatic measure of entropy and use it to measure the dynamical and topological aspects of RBN and LSM networks. 


\subsection{RELATED WORK}

\subsubsection{Cellular Automata}

Cellular Automata (CA) are simple homogeneous models of discrete dynamical systems that despite their simplicity may show complex dynamics. In the late 40's, John von Neumann [111] introduced CAs as a biologically inspired model of discrete-state discrete-time dynamical system. The simplest type of CA or Elementary CA (ECA) [49] consists of a one dimensional lattice of cells, each with a self-connection and two connections to its immediate right and left neighbors. Each cell may assume either of the two states "0" and "1." All cells change their states at the same time according to a binary function called the CA rule. The dynamical properties of this system depends on the initial configuration of the lattice and the CA rule.

Stephen Wolfram [114] pioneered the investigation in local and global dynamics of the ECA. He classified the space of CA rules into four classes. In finite time, CA rule classes I and II will end in a fixed-point or cyclic attractors from almost any initial configuration. Class III rules are very sensitive to initial configuration and will lead to the strange (chaotic) attractors. Class IV rules show complex dynamics and one of the rules, i.e., 110, has been proven to be computationally universal.

$\mathrm{Li}$ and Packard [67] studied the structure of the ECA rule space. They organized the 256 possible rules into 88 equivalence classes according to the internal symmetries in the rules. Based on the dynamics of the CA, they assigned each equivalence class to five different dynamical classes of (1) null dynamics, (2) fixedpoint, (3) periodic, (4) locally chaotic, and (5) chaotic. This assignment is not one-to-one and each rule may belong to many classes in this classification [68].

Wootters and Langton [115] studied the sharp transition in the dynamics of ECA rule space as a function of $\lambda$ (homogeneity) of the CA rules. The phase 
transition becomes sharp in the limit of infinite-valued CA. Increasing the number of local connections pushes this transition towards $\lambda=0$ which suggests that at infinite range neighborhood the transition vanishes. Langton [63] suggested that complex computation in ECA occurs at the region of the rule space that corresponds to the phase transition in $\lambda$. This region is called "the edge of chaos." However, Mitchell et al. [78,79] refuted the edge-of-chaos-computation argument by showing that it is possible to find $\mathrm{CA}$ rules in other regions of the rule space that perform complex computation.

The first method for the automatic design of CAs for parallel computation was proposed in [77]. This approach used genetic algorithms to evolve CA rules that could solve the density classification task. However, since this task requires global information processing it does not suit ECA dynamics since information transfer in ECA is limited. For finite size CAs, perfect solutions, i.e., CA rules that solve the task from all initial configuration of the CA, were not found. Analytical derivation of the CA rules and the initial configuration for the ECA to perform a desired configuration remains an open problem.

Tomassini et al. [110] investigated the dynamics and performance of ECA with a small-world topology [113], in which the lattice structure of the CA is randomly rewired into an irregular form. They showed that the small-world property increases the computational performance and pushes the dynamics of the ECA towards the critical phase. Small-world ECA are examples of dynamical systems with homogeneous functions and irregular interconnect. This irregular interconnect is characterized by existence of long distance links between nodes that are physically far apart. For more details about the network topologies see Section 1.3.4

\subsubsection{Random Boolean Networks}

Kauffman [52] introduced RBNs as a biologically viable model for gene regulatory networks. RBNs may be thought of as a generalized CA in which each node - out of 
the total of $N$-is connected in random to $K$ other nodes. Moreover, in RBN each node has a different transfer function that is randomly assigned. In other words, $\mathrm{RBN}$ is a heterogeneous counterpart of $\mathrm{CA}$, in which the connections are irregular and functions are heterogeneous. RBN is also called the NK model because one can define an ensemble of RBN using just two parameters $N$ and $K$. Kauffman himself studied many theoretical aspects of RBNs and their applications in the context of cell biology an homeostasis. [50,51,53-57,100,103]. Many authors have contributed to RBN and generalized the RBN into a model, in which the network nodes have an average of $\langle K\rangle$ connections per node [36,37].

Kauffman classified the dynamics of RBNs into three classes depending on the values of $\langle K\rangle$. For $\langle K\rangle<2$, the networks are likely to find a fixed point or periodic attractor quickly. Networks of $\langle K\rangle>2$ will have the chaotic dynamics, in which the networks will not find an attractor in finite time, or the the attractor will not have a finite size. At $\langle K\rangle=2$, the dynamical regimes of the networks show the maximal variance [56], this dynamical behavior is called the "edge of chaos." The median cycle length (number of states in the cyclic attractor) in ordered RBN scales according to $O\left(e^{\frac{1}{8} \log ^{2} N}\right)$, in the complex regime according to $O(\sqrt{N})$, and finally in the chaotic regime according to $O\left(0.5 \times 2^{N}\right)$. For small networks, the cycle length in complex regime scales with $N$ [19].

Derrida and Pomeau [30] devised an annealed approximation method for determining the dynamical regime of the RBNs as a function of $\langle K\rangle$ and $p$, i.e. the fraction of " 1 "s in the Boolean function of the nodes.

However, this result applies to a network at the thermodynamic limit, i.e., $N \rightarrow \infty$. For finite size networks, the Derrida criticality [30] is calculated by averaging the spreading of the two states of the network that are one Hamming distance apart after one time step, normalized by the network size [100]. If the result is equal to 1 , then the network is in the complex regime, if it is smaller than 1 , the network is in ordered regime, and if it is larger than one the network is in 
the chaotic regime.

Flyvbjerg [35] derived another order parameter for measuring complex dynamics of the network based on the frozen component. In this second method, a network is said to have complex dynamics if $50 \%$ of the nodes of the network change their state and the other $50 \%$ do not.

Many evolutionary approaches have been proposed that may bring any initial RBN to a critical dynamical regime $[22,40,55,70]$. The significance of critical dynamics in complex networks comes from the fact that many biological systems have been observed to operate at this regime $[15,28,29,56,60,64,106]$. RBN have also been used in task solving $[8,39,40,76]$. RBN has also been used as the reservoir in RC systems [102].

Serra et al. [96,97] studied the dynamics of power-law RBNs and found that these RBNs have fewer attractors than the classical RBNs. They also found that the transient length and the cycle periods of the attractors are significantly shorter in power-law RBNs. Darabos et al. [27] conducted a comprehensive study of dynamics of RBNs with Poissonian and power-law degree distribution under normal and noisy update rules.

Mesot and Teuscher [76] showed that Random Boolean Networks (RBN) outperform 1-D CA (with neighborhood as large as 7) in density classification. Moreover, they derived an analytical method to deduce the local rules in the network to perform the task. In this experiment, RBN also performs better than small-world CA [109].

\subsubsection{Liquid State Machines}

Liquid State Machines (LSM) were originally introduced as a model of cortical microcircuits in the brain [75]. The idea is that a system with rich intrinsic dynamics - called a reservoir - may remember the stimulations by an input signal. A memoryless output layer may then use the traces of this excitation in the 
reservoir and produces required output. Natschläger and Maass [81] studied basic information processing capabilities of LSM and found that networks that are able to compute the functions are the ones maximizing the information stored in the LSM. Bertschinger and Natschläger [18] extended Derrida's annealed approximation to calculate the spreading of perturbation in the LSM state that are due to changes in the input signals. They parameterized the network as a function of the connectivity $K$ and the variance of the weights of the connection matrix and illustrated that only the critical networks are able to differentiate between changes in the inputs. Boedecker et al. [21], in an attempt to find methods for initializing suitable LSM, introduced the average state entropy of the network as a measure of the complexity of the dynamics. Boedecker et al. postulated that a higher average state entropy results in higher LSM performance. They were able to tune the average state entropy by adjusting the spectral radius of the weight matrix. However, their experiment showed that the correlation between the average state entropy and the LSM performance is task-dependent. Büsing et al. [24] did a very extensive study on LSM. They parameterized the LSMs with the number of states per computing node, the connectivity, and the variance of the weight matrix. Then, they showed that for binary nodes, the performance is extremely sensitive to the connectivity and the sparsity of the weight matrix. As the number of states per node increases, the sensitivity to connectivity and sparsity in the LSM diminishes.

\subsubsection{Complexity and Heterogeneity in Networks}

Complex network science is concerned with the study of networks with many nodes that are connected in non-trivial ways [113]. The study of complex networks has recently gained much attention from several disciplines $[4,9,23,44]$ and the structure and function of these networks have been the subject of many studies $[20,31,41,64$, 82]. Complex networks may represent a wide variety of physical systems with large numbers of interacting parts, and thus they have been used to model many different 
real-world systems and phenomena [13]. Many interesting properties of complex networks have been attributed to their in-degree heterogeneity [105]. However, in the complex systems literature, heterogeneity refers to how close the network topology is to a star-like network $[3,38,106]$. This entails a scale-free in-degree distribution. The idea is that that there is no correlation between the node in-

degrees, i.e., a node with many connections might be connected to nodes with just a few connections. Moreover, in these networks there is a large difference between the number of connections of highly connected nodes and the rest of the nodes. Larremore et al. [64] has recently used a rigorous mathematical method to link the network topology to the dynamics of the network.

\subsection{MY CONTRIBUTIONS}

My contributions during the course of this thesis are:

1. Introducing entropy as pragmatic and generic measure of heterogeneity in three aspects of networks: node in-degrees, node functions, and interconnect irregularity.

2. Developing a formal framework for studying in-degree heterogeneity in a systematic way.

3. Developing a simulated annealing method for evolving in-degree with different heterogeneity levels.

4. Describing and formalizing the dynamics of the in-degree under evolution.

5. Formalizing the characteristics of the in-degrees with maximum heterogeneity levels.

6. Formalizing a framework for studying function heterogeneity.

7. Formalizing a framework for studying interconnect irregularity. 
8. Developing automated software framework for simulating LSM with controlled heterogeneity levels in in-degrees, functions, and interconnect.

9. Developing a high performance GPU-based software framework for studying RBN dynamics with controlled heterogeneity levels in in-degrees, functions, and interconnect.

10. Our GPU and MATLAB simulators includes 12000 lines of code.

11. Systematically studying network topology in networks with different heterogeneity in in-degrees and interconnect.

12. Systematically studying dynamics in RBN and LSM with different heterogeneity levels in in-degrees, node functions, and interconnect. 


\section{MODELS AND MEASURES}

\subsection{MODELS}

In the next two subsections we describe two types of random dynamical networks: RBN and LSM. We use the term random dynamical networks to refer to dynamical networks that are random in some sense, such as the functions of each node or the connectivity between the nodes. Since the networks have random connections, it is possible that some of the connections form recurrent loops, which result in time dynamics in the networks.

\subsubsection{Random Boolean Network (RBN)}

Overview. - Classical random Boolean networks [37] are simple discrete-time binary dynamical systems that are capable of modeling a wide variety of biological phenomena. Kauffman [52], Ashby [10] and many other researchers have used RBNs to model systems ranging from gene interaction to memory and brain functions. Although RBNs are a Boolean idealization of gene regulatory networks, they capture a lot of relevant behaviors in real gene regulation. We have previously showed $[39,108]$ that we can easily extend RBNs with inputs and outputs, and use them as building blocks for general purpose computing. In this context, we think of RBNs as a computing model for self-assembled nanoelectronics.

Structure. - The fundamental unit in a RBN is a node with $K$ input connections. At any instant in time, the node can assume either of the two binary states " 0 " or " $1 . "$ The node updates its state at time $t$ according to a $K$-to- 1 Boolean 
mapping of its $K$ inputs. Therefore, the state of a single node at time $t+1$ is only determined by its $K$ inputs at time $t$ and by one of the $2^{2^{K}}$ Boolean functions used by the node. Formally, a RBN is a collection of $N$ such binary nodes. For each node $i$ out of $N$ nodes, the node receives $K_{i}$ inputs, each of which is connected to one of the $N$ nodes in the network. In this model, self-connections are allowed.

The network is random in two different ways: 1) the source node for an input is chosen from the $N$ nodes in the network with uniform probability and 2) the Boolean function of node $i$ is chosen from the $2^{2^{K_{i}}}$ possibilities with uniform probability. Each node sends the same value on all of its output connections to the destination nodes. We denote the average connectivity in a RBN by:

$$
\langle K\rangle=\frac{1}{N} \sum_{i=1}^{N} K_{i}
$$

Because of the randomness in instantiating a RBN, it is common to talk about an ensemble of RBNs with $N$ nodes and average connectivity $\langle K\rangle$. This refers to all the instantiations of such RBNs. Once the network is instantiated, the collective time evolution at time $t$ can be described as follows:

$$
x_{i}^{t+1}=f_{i}\left(x_{1}^{t}, x_{2}^{t}, \ldots, x_{K_{i}}^{t}\right),
$$

where $x_{i}^{t}$ is the state of the node $i$ at time $t$ and $f_{i}$ is the Boolean function that governs the state update of the node $i$. The nodes are updated synchronously, i.e., all the nodes update their state according to a single global clock signal.

From graph theoretical perspective, a RBN is a simple directed graph with $N$ vertices and $L=\lfloor\langle K\rangle N\rfloor$ directed edges. Note that $L$ is an integer. Each of the $L$ edges are picked from the possible $N^{2}$ possible edges with uniform probability. This is the classic Erdös-Rényi random graph [33]. An important property of this class of random graphs is its binomial degree distribution:

$$
P(K=k)=\left(\begin{array}{c}
N \\
k
\end{array}\right) p^{k}(1-p)^{N-k} .
$$




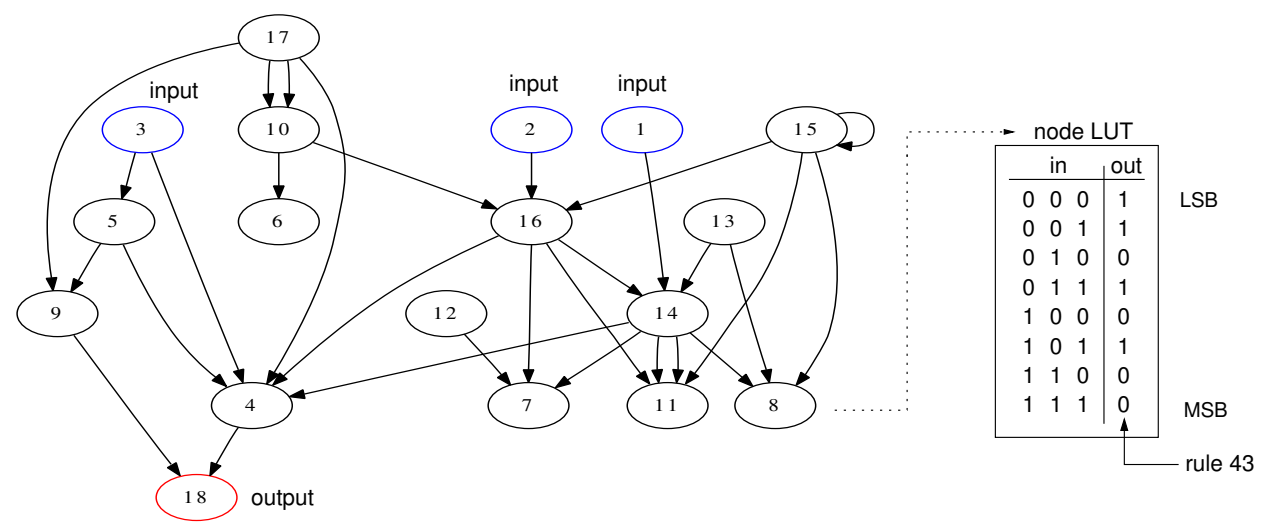

Figure 2.1: Example of RBN with 18 nodes and $\langle K\rangle=2$. Input signals are fed to the network via nodes 1, 2 and 3. One of the nodes (node 18 in this case) is chosen randomly to read the output.

Here, $P(K=k)$ denotes the probability of a node having $K=k$ incoming connections and $p$ is the probability of realization of an edge in the graph. Kauffman's original model assumes a static environment and therefore Kauffman did not introduce any exogenous inputs to the network [52]. To compute with RBNs, we introduced $I$ additional input nodes that distribute the input signals to the nodes in the network $[39,108]$. These $I$ nodes are not counted as part of the $N$ nodes in the RBN. However, the source node of $K_{i}$ links for each node $i$ is randomly picked from $N+I$ nodes with uniform probability. The average connectivity is calculated according to Eq. 2.1. Note that the $I$ input nodes themselves do not have any inputs and are not counted in calculating $\langle K\rangle$. The output of the system is read from one of the $N$ nodes that is chosen with equal probability. To avoid confusion, we generally refer to our extension with the term Boolean Network (BN) instead of RBN.

System dynamics. - From the deterministic update of each node together with the synchronous update in a RBN, it follows that the RBN as a whole also behaves deterministically; the state of the network at time $t+1$ is only determined 
by the state of the network at time $t$. A RBN is made up of $N$ binary nodes, therefore, the behavior of the system is a trajectory in the $N$ dimensional state space with $2^{N}$ possible states. It is possible that different trajectories merge after some time, but a single state at time $t$ only maps to a single state at time $t+1$. Because of the finite-size state space, the system eventually revisits a state. From then on, the system enters a cycle of $\Gamma$ states that repeat in time. $\Gamma$ is also called the cycle length. The cycle is called an attractor in the language of dynamical systems. All the states that the system visits before it enters the attractor are called transient states. There are many different transient trajectories that can lead to the same attractor. The subspace of all the $2^{N}$ states that lead to an attractor together with the $\Gamma$ attractor states are called a basin of attraction.

\subsubsection{Liquid State Machine (LSM)}

Overview. - Reservoir Computing (RC) $[47,75]$ is a computational paradigm which promotes universal computation using transient dynamics, as opposed to stable states. The basic idea behind RC is to employ a computational core, the reservoir, which is operating in a proper dynamical regime to perform temporal computation, e.g., tracking or control of a dynamical signal. The RC paradigm is particularly attractive as a model for unconventional computing systems because it does not make any assumption about the nature of the reservoir network. In principle, the reservoir can be any system with sufficiently complex dynamics. This means that any molecular or nano-scale system can in principle be used to build the reservoir. Figure 1.3.3 shows a schemaitc for an LSM with input and readout layers.

Structure. - The reservoir is the heart of the RC paradigm. In LSM, the reservoir is a collection of $N$ interconnected nodes. Unlike in $\mathrm{RBN}$, the state of a node in LSM is represented by a real number. Each node in the reservoir has $K$ incoming connections with real valued weights. The reservoir is random in two 
ways: 1) the selection of connection weights and 2) source node of a connection. The weights are identically and independently distributed from a normal distributions with mean 0 and variance $\sigma^{2}$ denoted by $\mathcal{N}\left(0, \sigma^{2}\right)$. The source of $K$ inputs of a node is selected from the $N$ nodes in the reservoir with uniform probability. Each node also receives a connection from one of the $I$ input signals connected to the reservoir. The state of the node $i$ at time $t$ is denoted by $x_{i}^{t}$ and is updated in discrete time steps as follows:

$$
x_{i}^{t+1}=\tanh \left(\sum_{j=1}^{N} w_{i j} x_{j}^{t}+\sum_{k=1}^{I} u_{k}^{t}\right) .
$$

The weight of the connection from node $j$ to node $i$ is denoted by $w_{i j}$ and $u_{k}^{t}$ is the value of the input signal $k \in\{1, \ldots, I\}$ at time $t$. All the nodes in the reservoir use the same equation to update their state synchronously. For online computation, the reservoir is extended by a separate readout layer with $O$ nodes. Each node in the readout layer is connected to each node in the reservoir. The output of the node $o$ in the readout layer at time $t$ is denoted by $y_{o}^{t}$ and is computed according to:

$$
y_{o}^{t}=\operatorname{sign}\left(\sum_{j=1}^{N} \alpha_{j} x_{j}^{t}+b\right) .
$$

Parameters $\alpha_{j}$ s are the weights on the inputs from node $j$ in the reservoir to node $o$ in the readout layer and $b$ is the common bias for all the readout nodes. Parameters $\alpha_{j}$ and $b$ can be trained using a pseudo-inverse regression to realize a target output [45]. In a variation of the LSM called the Echo State Machine (ESM) the nodes in the readout layer are connected to all the reservoir nodes as well as the input nodes, and they have recurrent connections to and from other nodes in the readout layer too [45-48].

System dynamics. - The dynamics of the reservoir forms a continuous trajectory in a $N$-dimensional state space. The perturbation on the transient dynamics of the reservoir by the input signals can be interpreted by the readout layer to 


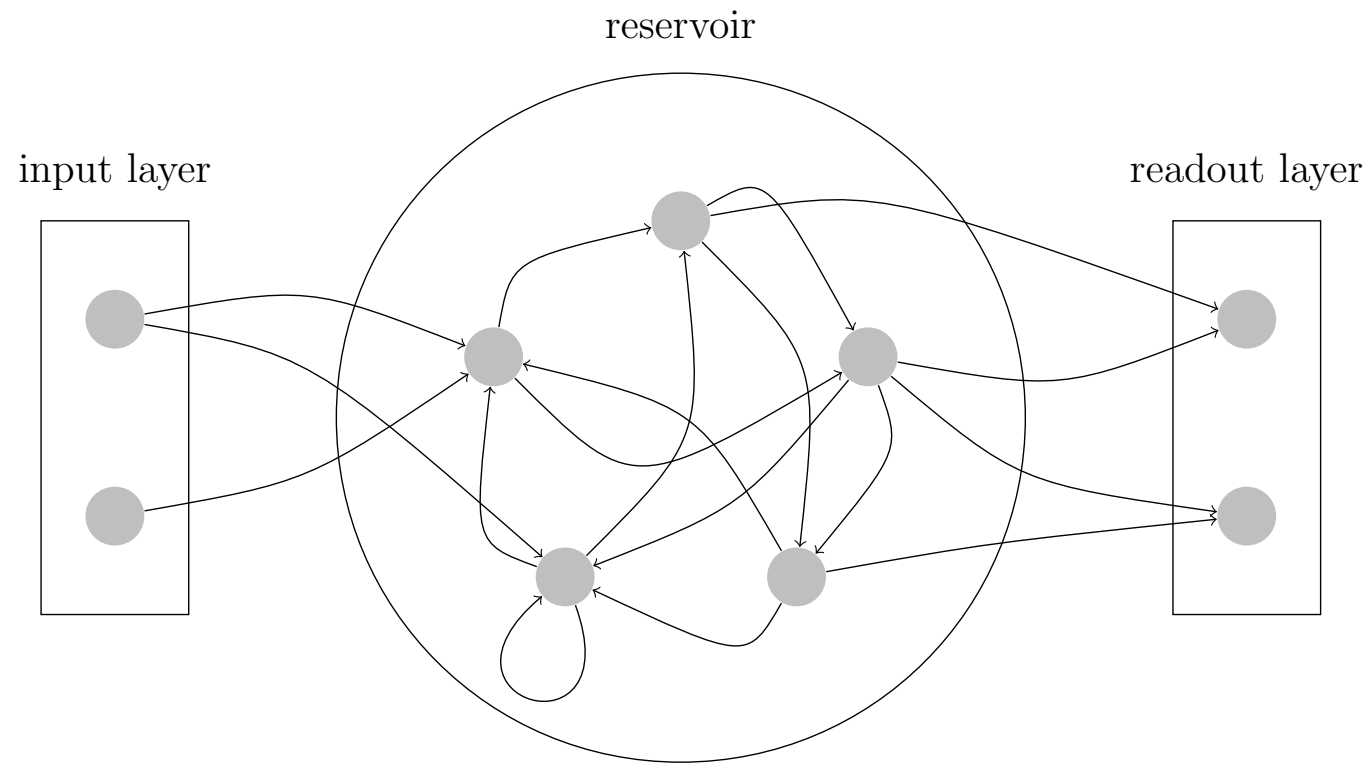

Figure 2.2: Schematic of a reservoir computing system. The input layer delivers the input signals to random nodes inside the reservoir. The readout layer receives output signals from random nodes inside the reservoir. The reservoir itself is made of a collection of computing nodes that are randomly interconnected. The reservoir creates a representation of the input signals that can be read and classified by the readout layer. Learning is performed by training only the readout layer nodes and connections. 
produce a desired target function. In Section 1.3.3 we review some of the related work that has been done on LSM.

\subsection{ENTROPY AND HETEROGENEITY}

The measurement of entropy is equivalent to the problem of diversity in a population of objects. Rosca [93] used entropy to measure the population diversity in the context of artificial evolution and showed that entropy is a pragmatic measure of the diversity of a population. For a generic set of objects $A$ with $n$ total elements of $m$ distinct classes, we calculate the entropy as follows:

$$
\mathcal{H}\left(P_{A}\right)=-\sum_{i=1}^{m} p\left(x_{i}\right) \log _{2}\left(p\left(x_{i}\right)\right) .
$$

$P_{A}$ is the probability distribution of the objects in set $A$ and $p\left(x_{i}\right)$ are the probability of occurrence of each distinct element $x_{i} \in A$. We use entropy as a measure of heterogeneity in a sample of numerical objects and justify its use instead of more common measures of diversity, such as standard deviation and variance. Our approach is to use entropy as an objective measure of observable diversity in a collection of numerical objects. We call entropy an objective measure since its value is independent of the values of the numerals in the sample. We say entropy is a measure of observable diversity to differentiate it from simply the number of different numbers in the sample. Note that although here we use entropy to measure

the heterogeneity in the nodes' in-degree of a network, entropy is general enough to be used on a sample of objects that are not numerals.

Heterogeneity in a sample of objects refers to how many different classes of objects there are in the sample, i.e. $m$. However, it is misleading to use $m$ as a measure of heterogeneity because some of the objects might be rare and not be observed as frequently. For example, let set $A$ be a collection of 100 numbers with 99 "0" and one "1." $A$ contains two different numbers, i.e., $m=2$. But if we randomly pick five numbers from $A$, we are mostly going to pick "0" s. In fact, if 
we repeat several times, we are mostly likely to see all "0"s every time. Therefore, although we really have two different numbers, it is difficult to see them in our random samples. The observable number of classes $m$ is really much closer to one rather than two. This observable heterogeneity is exactly what entropy measures. Entropy measures this observable heterogeneity by weighing different numbers by their probability of occurrence in the sample. Another way of measuring the heterogeneity in a sample of numerals is through measuring the variance or deviation from the mean of the sample using the variance $\left(\sigma^{2}\right)$ or the standard deviation $(\sigma)$. This approach is however impractical to use for a generic measure of heterogeneity because $\sigma$ can only be used with a set of numbers and its value depends on the values of the numbers.

The standard deviation of a set of $n$ numbers $A$ with mean $\mu$ is given by Eq. 2.7. The value of the standard deviation therefore depends on the scale of the numbers in $A$, not just the diversity of them. Variance suffers from the same problem since it is just $\sigma^{2}$.

$$
\sigma=\sqrt{\frac{1}{n} \sum_{i=1}^{n}\left(x_{i}-\mu\right)^{2}} .
$$

The entropy of a $A$, however, solely depends on the probability of the observations of individual numbers in the set, and not the numerical values. Moreover, even if the set under study has other objects than numerals, we can still calculate the probability of each class of objects in the set.

Figure 2.11(b) shows the entropy and standard deviation of a sample of 100 numbers drawn from uniform and normal distributions. Although the distributions in Figures 2.3(a) and 2.3(c) are identical to Figures 2.3(b) and 2.3(d) respectively, the values of the standard deviation $(\sigma)$ varies because the values are in different orders of 10 , i.e. $1,10,100, \ldots$ The entropy $(\mathcal{H})$ of the distributions is scaleinvariant however. In the next section, we see how we can apply entropy to the in-degree distribution of a network to measure in-degree heterogeneity. 

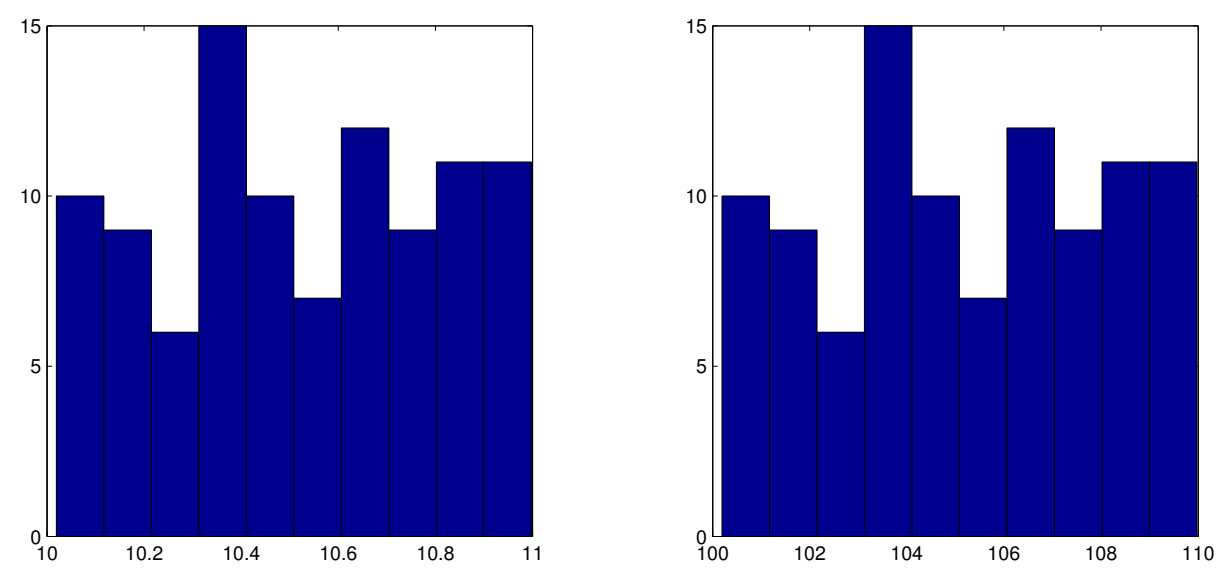

(a) $A=\left\{x_{i} \mid 10<x_{i}<11\right\}$, uniform. (b) $A=\left\{x_{i} \mid 100<x_{i}<110\right\}$, uniform. $\sigma=0.28377$ and $\mathcal{H}\left(P_{A}\right)=6.64$. $\sigma=2.8377$ and $\mathcal{H}\left(P_{A}\right)=6.64$.
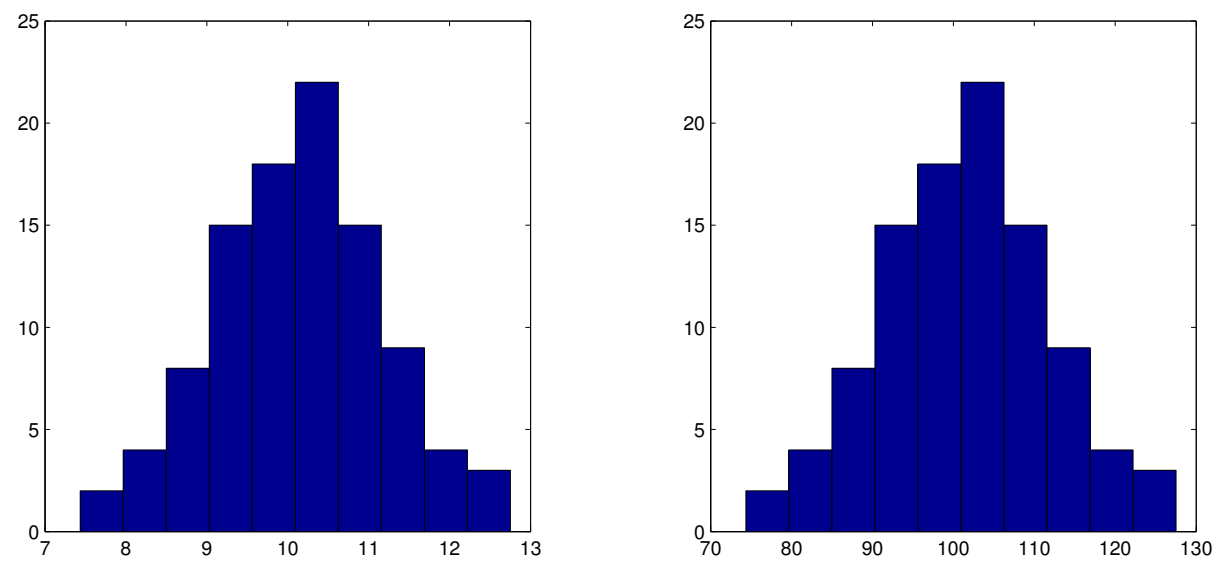

(c) $A=\left\{x_{i} \mid 7<x_{i}<13\right\}$, normal. (d) $A=\left\{x_{i} \mid 70<x_{i}<130\right\}$, normal. $\sigma=1.0601$ and $\mathcal{H}\left(P_{A}\right)=6.64$. $\sigma=10.6008$ and $\mathcal{H}\left(P_{A}\right)=6.64$.

Figure 2.3: The standard deviation $(\sigma)$ and the entropy $H$ of a sample of 100 numbers taken from uniform and normal distributions. We see in the example of the uniform and normal distributions that the same distribution on different values result in different $\sigma$, but the same $\mathcal{H}\left(P_{A}\right)$. However, different distributions might have the same value of $\mathcal{H}\left(P_{A}\right)$, which means we need to control for the distribution in our study. 


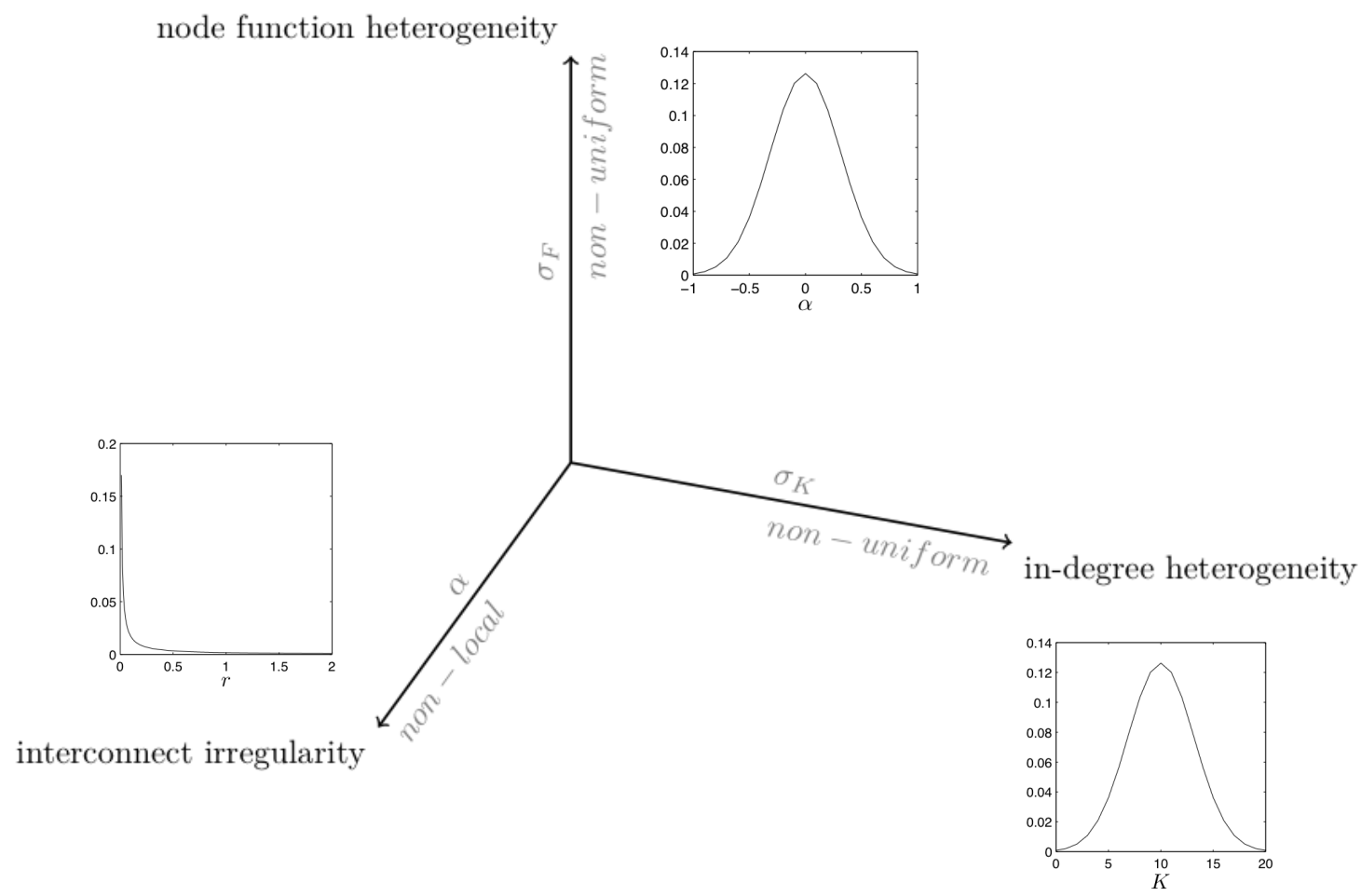

Figure 2.4: The parameter space we will use for our study include: the power-law exponent of the connection length distribution $\alpha$, entropy of the exponential indegree distribution, and the standard deviation of the Gaussian distribution for the coefficient of the hyperbolic tangent function $\sigma_{F}$.

We can study heterogeneity in network systems in at least three different dimensions:

1. Connectivity per node

2. Node function

3. Locality of connections between nodes

Figure 2.4 illustrates these aspects of heterogeneity in network systems. In the following, we will describe what in-degree heterogeneity means in our study. We 
will develop a formal framework, which allows us to create a homogeneous indegree and evolve it through many time steps to reach an in-degree with various levels of heterogeneity.

\subsection{IN-DEGREE HETEROGENEITY}

One aspect of structure in a network is the in-degrees of the nodes on the network. Figure 2.5 shows a simple directed network in which each node has two inputs $K=$ 2. Number of inputs in a node is called the in-degree of that node. The network nodes are arranged in a 2-dimensional lattice that extends in four directions. To describe the in-degrees of the network one would create a sequence of the in-degrees of the nodes in the order of indices given to the nodes. This in-degree sequence can be converted to a frequency distribution, which is the in-degree distribution of the network.

The network on Figure 2.5 is an example of a network with homogeneous or uniform in-degrees, i.e, all the nodes have the same in-degree $\langle K\rangle=2$ and the frequency distribution of the in-degrees only has one non-zero element. In networks in which the in-degree distribution is non-uniform, different nodes assume different number of inputs and therefore the in-degree sequence will be a sequence of many different numbers. We say the in-degree distribution of such network is heterogeneous. Figure 2.6 shows an example of such a network.

Many authors have studied the properties of networks with different in-degree distributions $[12,32,59,84,85,113]$. The in-degree distribution strongly affects the topological properties of the network and results in small-world [113] and scalefree [12] phenomena, in addition to influencing dynamical processes that may occur on the network.

During the course of this thesis, we developed a framework to produce networks with particular in-degree heterogeneity. In this section, we first introduce and justify the use of entropy as a measure of in-degree heterogeneity. We then explain 


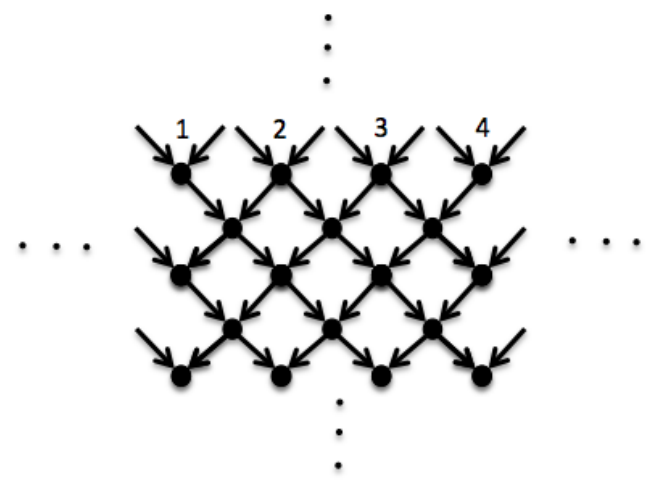

In-degree sequence: $\quad 2,2,2,2,2, \ldots$

homogeneous/uniform indegree

Figure 2.5: A network with homogeneous or uniform in-degrees, where each node has two inputs $\langle K\rangle=2$. The numbers on the first row are the given node indices. In-degree sequence is the sequence of in-degrees ordered according to the indices of the nodes in the network. In-degree distribution refers to the frequency distribution of the in-degrees in the network.

the challenges in the systematic study of in-degree heterogeneity and introduce the framework we developed.

From here on, we use the entropy when we talk about the heterogeneity in in-degrees of nodes in a network as follows. For a directed network $G=\{V, E\}$, where $V$ is the set of nodes in the network and $E$ is the set directed links between the nodes, we define the degree sequence $\psi$ as the set of the in-degrees for nodes in $V$. We indicate the entropy of $\psi$ by $\mathcal{H}\left(P_{\psi}\right)$, where $P_{\psi}$ is the probability distribution that the node $i$ has in-degree $K_{i}$. For any distribution, the value of the entropy is maximum if the values are uniformly distributed. The minimum value of the entropy of a distribution is 0 and occurs in a distribution in which only one possible value is realized. For example, if all the values in set $A$ are "1," then the entropy of the set $A$ is $\mathcal{H}\left(P_{A}\right)=0$ bits. If the set is $A=\{1,2, \ldots, 100\}$, then $\mathcal{H}\left(P_{A}\right)=6.6439$ 


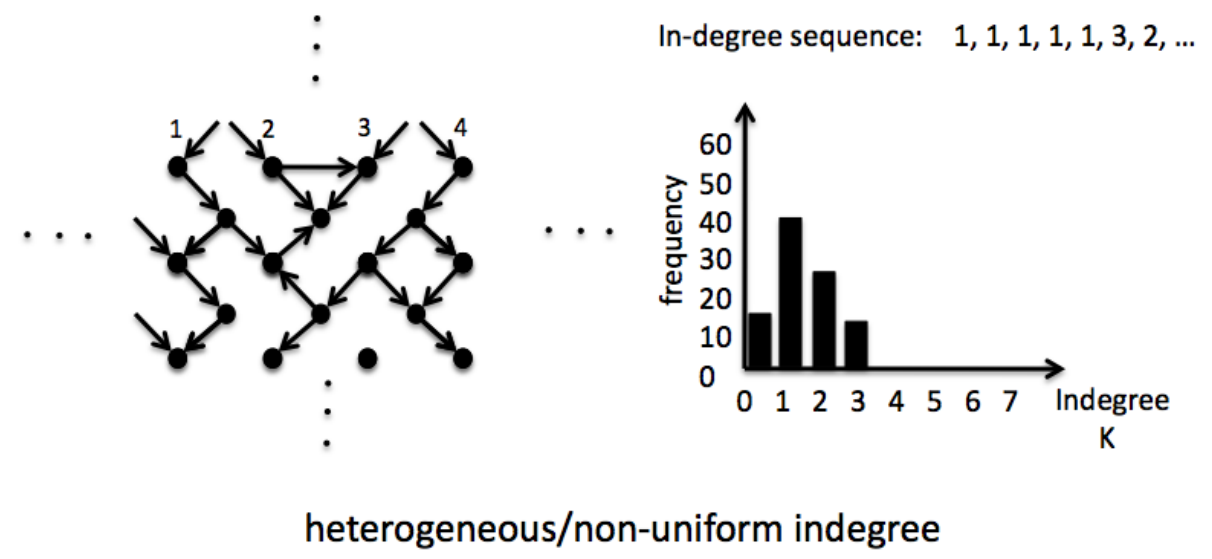

Figure 2.6: A network with heterogeneous (or non-uniform) in-degrees, where each node $i$ has a different number of inputs $K_{i}$. The numbers on the first row are the given node indices. In-degree sequence is the sequence of in-degrees ordered according to the indices of the nodes in the network. In-degree distribution refers to the frequency distribution of the in-degrees in the network.

bits.

In section 2.3.1 we discuss the problem in a controlled study of $\mathcal{H}\left(P_{\psi}\right)$ and its possible ramifications. We will explain that choosing a distribution is necessary when we study the properties of networks with various $\mathcal{H}\left(P_{\psi}\right)$. However, we are interested in studying the effects of heterogeneity of in-degree distribution on the network dynamics, which is highly influenced by the average connectivity of the network. Therefore, as we change the heterogeneity in the network, the average connectivity should remain fixed. As we will see, changing the entropy while controlling the mean of a distribution is a non-trivial problem.

\subsubsection{Entropy and In-degree Distribution}

A systematic study of the network dynamics as a function of $\mathcal{H}\left(P_{\psi}\right)$ imposes a challenge on the way we can construct our experiments. The dynamics of network 
are heavily influenced by the average connectivity per node $\langle K\rangle$ in the network (see Chapter 1.3.2). Therefore, in our study of network dynamics, $\langle K\rangle$ is one of the parameters that needs to be controlled.

In other words, we need to choose a model for our in-degree sequence $\psi$ as to control the entropy $\mathcal{H}\left(P_{\psi}\right)$ and the connectivity $\langle K\rangle$ independently. A Gaussian distribution is the only distribution which allows us to vary the entropy and the mean of the distribution independently. The entropy of a Gaussian distribution depends only on the standard deviation of the distribution. Since in-degrees in a network are all positive integers, moving away from a completely homogeneous in-degrees with entropy 0 to a completely heterogenous in-degrees in a uniform distribution without changing the mean becomes impossible. A pragmatic approach is to start from a network with homogenous in-degrees and incrementally change the in-degrees using a stochastic hill climbing algorithm to maximize the entropy [61]. We then use the snapshots of the in-degrees throughout the hill climbing to use in our experiments. Similar approaches have been used to study the small-world and spatial networks $[62,87,113]$. We use the configuration model [85] to sample the graphs with an arbitrary in-degree sequence and study their dynamics and other properties. This is done by first creating an in-degree sequence and randomly choosing source nodes for each node in the in-degree sequence according to their predefined in-degree.

In the next section, we will propose a model to systematically create in-degree sequences with various entropies. This model treats the in-degree sequence itself as a dynamical system. Section 2.3.2 describes the details of our simulated annealing algorithm. We will later see the consequences of this approach and its connection to other physical phenomenon studied in statistical physics. 


\subsubsection{In-degree Sequence as a Dynamical System}

In this section, we describe the details of the dynamical model that we will use to generate in-degree sequences with various entropies. This model is dynamical in the sense that the object of the model, the in-degree sequence, changes in time to maximize the entropy. We previously explained that to study in-degree heterogeneity, we need a dynamical model to generate in-degree sequences starting with zero entropy and evolve the sequence towards the maximum possible entropy. The object that undergoes the time evolution here is the in-degree sequence $\psi$. This is a sequence of in-degrees for each node in a network with $N$ nodes and $L$ links $\psi=\left\{x_{i} \mid 0<x_{i}<K_{\max }, 1<i<N\right\}$. If the network has an average connectivity $\langle K\rangle=K$, that is integer, the starting condition of the process is where all the nodes in the network have the same in-degree and thus $\psi=\{K, K, \ldots, K\}$. This sequence correspond to a class of networks with completely homogeneous indegrees, and thus $\mathcal{H}\left(P_{\psi}\right)=0$. We form a partition function for our simulation as follows:

$$
Z=\sum_{\{\psi\}} e^{-\frac{\mathcal{H}\left(P_{\psi}\right)}{T}} .
$$

$\mathcal{H}\left(P_{\psi}\right)$ takes the form of a Hamiltonian, which will be evaluated for the set of all the possible in-degree sequences $\{\psi\} . \psi$ is the in-degree sequence, which is the dynamical variable, we will be sampling during our simulations to generate in-degree sequences we need for our study. Each simulation step consists of:

1. Mutate $\psi$ by randomly choosing a node with positive in-degree and decreasing its in-degree by 1 .

2. Randomly choosing another node with in-degree $<K_{\max }$ and increasing its in-degree by 1 .

3. Evaluate the new $\mathcal{H}\left(P_{\psi}\right)$ and calculate $\Delta \mathcal{H}\left(P_{\psi}\right)$. 
4. If $\delta \mathcal{H}\left(P_{\psi}\right) \geq 0$, accept the new sequence, otherwise accept the sequence with probability $e^{-\frac{\delta \mathcal{H}\left(P_{\psi}\right)}{T}}$.

This process is equivalent to the Metropolis algorithm for Monte Carlo simulations [61] and samples the space of $\psi$ without bias to generate a series of in-degree sequences with increasing entropy. The assumption here is that the system under time evolution is ergodic and eventually reaches an equilibrium. We will see in the next section that the in-degree sequence behaves as expected and therefore this methodology is valid in this study. In Section 2.3.3 we will show the results of this simulation and examine the generated sequences more closely. We will see that in fact the process will lead into a stationary state in entropy and distribution of the in-degrees.

\subsubsection{Evolution of In-degree Sequence}

To systematically study the heterogeneity of in-degrees in the network and its effects on the dynamical properties of the networks, we defined the entropy of the in-degree sequence of the network. In addition we proposed a simple model which lets us generate a series of in-degree sequence with increasing entropy. The process takes an initial in-degree sequence of $N$ nodes with a specified average connectivity $\langle K\rangle$ and treats the sequence as a thermodynamical system undergoing time evolution towards equilibrium state at maximum entropy.

Figure 2.7(a) illustrates the value of the $\mathcal{H}\left(P_{\psi}\right)$ as the sequence undergoes time evolution towards maximum entropy. The process rapidly increases entropy and reaches stationary state at which the value of the entropy fluctuates around a mean close to the maximum entropy for an ideal truncated exponential distribution with mean 2 (see Section 2.3.5). This maximum value is $\approx 2.7$. The fluctuation in the final value is due to the probabilistic nature of the mutation moves during the time evolution at finite temperature. At high temperature this fluctuations maximizes due to equal probability of the accepted mutations. To reach a truly stationary 


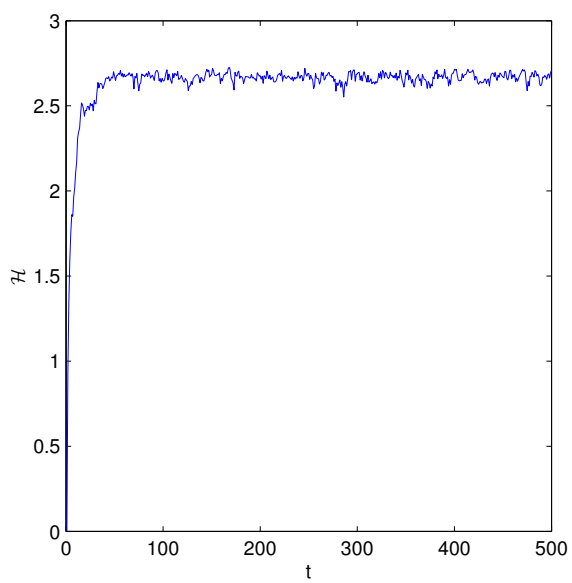

(a) $T>0$

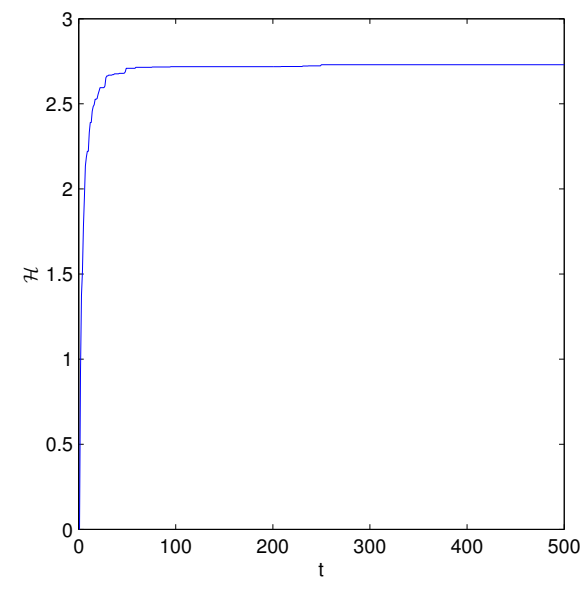

(b) $T=0$

Figure 2.7: (a) Entropy of in-degree distribution of the network that is undergoing random mutation. The plot corresponds to a single run. We see a rapid increase followed by a fluctuating stationary period, which corresponds to the equilibrium in-degree distribution. In this equilibrium the fluctuations in the entropy is due to random nature of the mutation, but on average the value of the entropy is constant and its trend is stable. The average in-degree $\langle K\rangle=2, N=100$, and average maximum value of the entropy reached is $\mathcal{H}_{\text {max }}=2.7073$. (b) Entropy of in-degree distribution of the network that is undergoing random mutation at low temperature $T=0$. We see a rapid increase followed by a constant entropy period, which corresponds to the equilibrium in-degree distribution. The average in-degree $\langle K\rangle=2, N=100$, and average maximum value of the entropy reached is $\mathcal{H}_{\max }=2.7299$. 
entropy value, we may use a very low temperature, i.e., $T=0$. Figure 2.7(b) shows the same experiment with zero temperature leading to a stable value of the entropy.

A peculiar aspect of these results is that the maximum entropy achieved in the simulation is lower than the expected entropy of a uniform distribution, where the values may vary between 0 and $K_{\max }$. In our simulations we set $K_{\max }=10$. This means that the maximum value for the entropy for a uniform distribution should be $\mathcal{H}_{\text {max }}=\log _{2}(10)=3.32$. However, in our simulations we observe that $\mathcal{H}_{\text {max }} \approx$ 2.7. In section 2.3.4, we will see that this discrepancy is due to thermodynamic effects that gives rise to exponential in-degree distribution instead of of the uniform distribution.

\subsubsection{Maximum Entropy Distribution}

The dynamical model we proposed to maximize the entropy of the in-degree sequence of the network results in sub-optimal values (see Figure 2.7(b) and Section 2.3.3). We know the highest entropy for any distribution is when the distribution is as flat as possible (see Section 2.3.1 and Section 2.2). We would naturally expect to see a uniform distribution if we convert the in-degree sequence after the evolution to its frequency distribution. On the contrary, when we look at the in-degree distribution after the evolution, we see a very rapidly decreasing distribution (Figure 2.8) that is very close to a standard exponential distribution of the form:

$$
P(x)=\lambda e^{-\lambda x} .
$$

The parameter $\lambda$ is the inverse of the mean of the distribution. The standard exponential distribution is defined on the support set $S=[0, \infty)$. To calculate the entropy of this distribution we take the expected value of the $\log _{2}(P(x))$ as follows: 


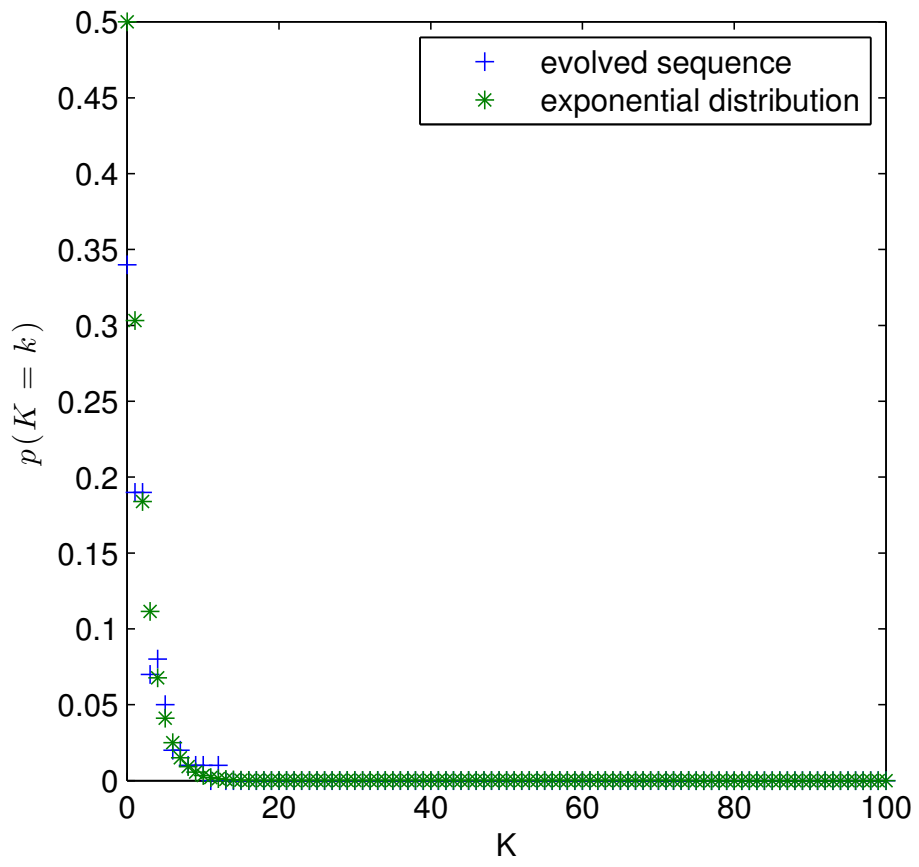

Figure 2.8: Equilibrium in-degree distribution for maximum entropy networks after evolution. $\langle K\rangle=2$. The resulting distribution looks similar to an exponential distribution. 


$$
\begin{aligned}
\mathcal{H}\left(P_{\psi}\right) & =-\left\langle\log _{2} P(x)\right\rangle \\
& =\int_{0}^{\infty} P(x) \log _{2} P(x) d x \\
& =\int_{0}^{\infty} \lambda e^{-\lambda x} \log _{2}\left(\lambda e^{-\lambda x}\right) d x \\
& =1-\log _{2} \lambda .
\end{aligned}
$$

The entropy of the distribution for $\langle K\rangle^{-1}=\lambda=\frac{1}{2}$ is $\mathcal{H}\left(P_{\psi}\right)=1-\log _{2}\left(\frac{1}{2}\right)=2$. This value is still significantly different from our calculation of $\mathcal{H}_{\max } \approx 2.73$ as shown in Section 2.3.3.

The difference between the entropy of the evolved distribution and the entropy of the standard exponential distribution is due to the known effects of truncated exponential distribution [43]. In our case, the support for the truncated exponential is $S=\left\{x \mid 0 \leq x \leq K_{\max }\right\}$. The truncated exponential distribution is defined as follows:

$$
P(x)= \begin{cases}a e^{-b x} & : x \in\left[0, K_{\max }\right] \\ 0 & : \text { otherwise }\end{cases}
$$

The parameters $a$ and $b$ must be calculated by fitting to empirical data. Since this distribution is an empirical distribution, the solution to the maximum entropy problem must be investigated through simulation. This not only does not change the validity of the framework that we proposed here to study the heterogeneity in networks, but also supports the fact that this study must be carried out experimentally and is beyond any analytical analysis. We will study the parameters of the truncated exponential and the entropy of the truncated exponential distribution as a function of $\langle K\rangle$ and $K_{\max }$ in Section 2.3.5.

\subsubsection{Truncated Exponential Distribution}

For our purposes, we need to know if the entropy value is a predictable quantity as a function of the number of mutations in the system. We define the truncated 
exponential distribution over the support set $S=\left[0, K_{\max }\right]$ as follows:

$$
P(x)= \begin{cases}a e^{-b x} & : x \in\left[0, K_{\max }\right] \\ 0 & : \text { otherwise }\end{cases}
$$

To understand the behavior of this distribution we have to fit the parameters $a$ and $b$.

$K_{\max }$ is a reasonable limit for the maximum connections per node and depends on the particular application. Many studies that use RBN or other dynamical networks use a maximum number of connections per node for two reasons: restricting the size of the look-up table of RBN [92] and limitations in the number of connections per node due their maintenance cost in biologically inspired networks [7]. We will need these results to have a formal way of predicting the shape of the in-degree distribution in maximally heterogeneous systems. We will use the procedure described in Section 2.3.2 to maximize the heterogeneity of the in-degree distributions with average in-degree of $0<\langle K\rangle<8$ and $8 \leq K_{\max } \leq 16$. We fit the exponential distribution to the data from the maximum entropy distribution from our simulations and calculate $a, b$, and the entropy $\mathcal{H}\left(P_{\psi}\right)$ for these final distributions. We denote the exponential parameters using $a\left(\langle K\rangle, K_{\max }\right)$ and $b\left(\langle K\rangle, K_{\max }\right)$.

Figure 2.9(a) shows the interpolant fit of the average calculated, i.e., $a\left(\langle K\rangle, K_{\max }\right)$. In exponential distributions, $a\left(\langle K\rangle, K_{\max }\right)$ is the probability of $K=0$, i.e., number of nodes with no input connections. In terms of the structure of the networks, $a\left(\langle K\rangle, K_{\max }\right)$ directly determines the size of the frozen component $[35,53]$ in the networks. The frozen component of the network are the nodes with fixed values as the network runs. Indirectly, $a\left(\langle K\rangle, K_{\max }\right)$ also determines the size of the relevant component $[14,58]$, i.e., the number of nodes that are mutually reachable from one another through one or more transient nodes, and therefore affect their own state over time. It is expected that for sparse networks, i.e., as $\langle K\rangle$ decreases, the frozen component grows and the connected component shrinks. Moreover, we see that 


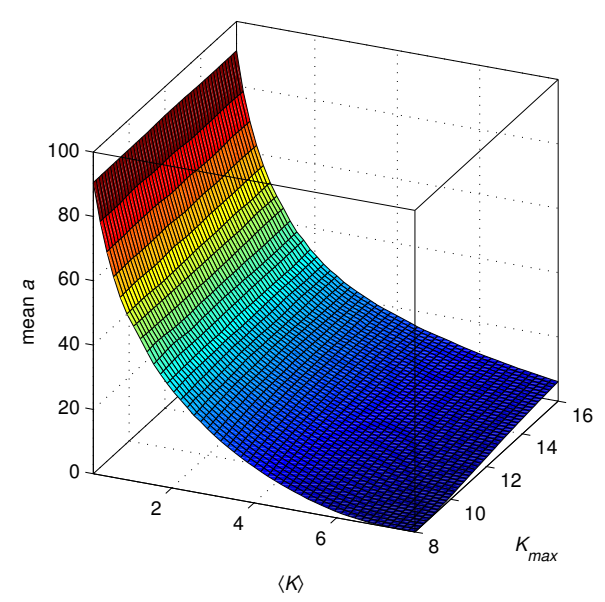

(a) mean $a$

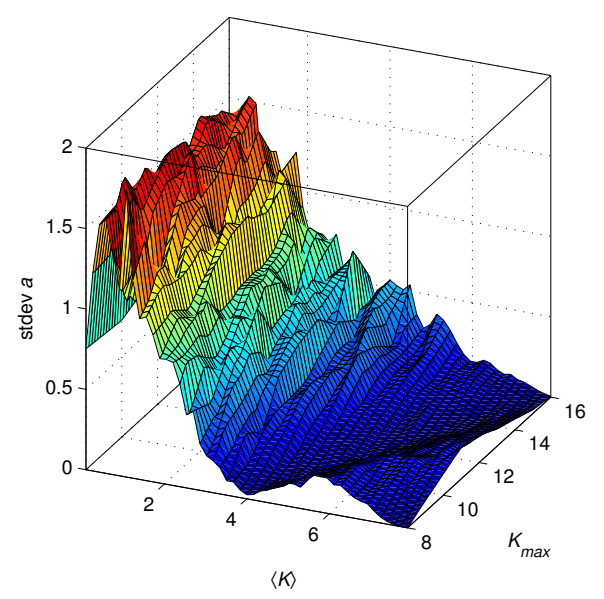

(b) standard deviation of $a$

Figure 2.9: The mean and standard deviation of the coefficient $a$ in the truncated maximum entropy distribution of the form $a e^{-b x}$.

an increase in $K_{\max }$ also results in an increase in $a\left(\langle K\rangle, K_{\max }\right)$. This is because smaller $K_{\max }$ force the mutation process to distribute the links in the network more evenly. As $K_{\max }$ increases, however, some nodes may attract more connections and therefore the number of connections to be distributed among the rest of the nodes decreases. To determine how accurate the values of $a\left(\langle K\rangle, K_{\max }\right)$ are, we plot the standard deviation of these values $\sigma_{a}\left(\langle K\rangle, K_{\max }\right)$ in Figure 2.9(b). We observe that for sparse networks with connectivity $\langle K\rangle<4$, the most predictable values of $a\left(\langle K\rangle, K_{\max }\right)$ with standard deviation zero occurs at $\langle K\rangle=\frac{K_{\max }}{2}$.

Next, we investigate the values of the decay $b\left(\langle K\rangle, K_{\max }\right)$. This decay values determine the shape of the exponential distribution after the maximization of the entropy. In particular, $b\left(\langle K\rangle, K_{\max }\right)$ determines how sharply the values of the distribution decreases, which results in lower entropies $\mathcal{H}\left(P_{\psi}\right)$. Figure 2.10(a) shows $b\left(\langle K\rangle, K_{\max }\right)$. For a large interval of connectivity $2<\langle K\rangle<6$ and maximum in-degree $K_{\max }>10, b\left(\langle K\rangle, K_{\max }\right)$ does not show any abrupt change, meaning that the shape of the underlying in-degree distribution and therefore its entropy 


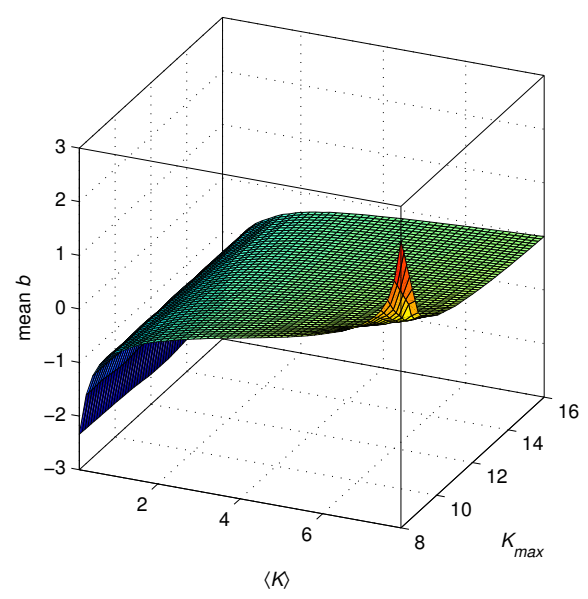

(a) mean $b$

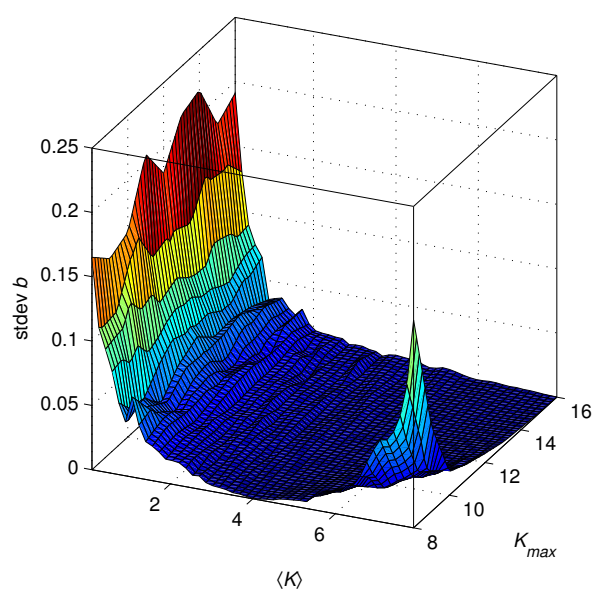

(b) standard deviation of $b$

Figure 2.10: The mean and standard deviation of the coefficient $b$ in the truncated maximum entropy distribution of the form $a e^{-b x}$.

$\mathcal{H}\left(P_{\psi}\right)$ does not vary abruptly. The value of $b\left(\langle K\rangle, K_{\max }\right)$ also shows very small fluctuations (Figure 2.10(b)). General properties of the the entropy of the maximally heterogeneous in-degree distribution can be derived from the behavior of $b\left(\langle K\rangle, K_{\max }\right)$. For $\langle K\rangle<2$, we expect to see very low entropy values, meaning that very sparse networks have invariably low in-degree heterogeneity. For the regions of $b\left(\langle K\rangle, K_{\max }\right)$ where the values are stable and positive, we expect high values of the entropy of the in-degree distribution and therefore maximum heterogeneity in the in-degrees. As $\langle K\rangle \rightarrow K_{\max }$, the value of $b\left(\langle K\rangle, K_{\max }\right)$ peaks sharply. This implies a sharp decay in the underlying in-degree distribution resulting in very low entropy and therefore low heterogeneity.

Figure 2.11(a) shows $\mathcal{H}\left(P_{\psi}\right)$ for the maximum entropy exponential distribution, i.e., after the mutation of the in-degrees as a function of $\langle K\rangle$ and $K_{\max }$. This result is in agreement with our expectation from the values of $b\left(\langle K\rangle, K_{\max }\right)$. For $K_{\max }<$ 10, we observe large changes in the heterogeneity of the in-degree distributions as a function of $\langle K\rangle$. In fact, the entropy looks like an inverse quadratic with its 


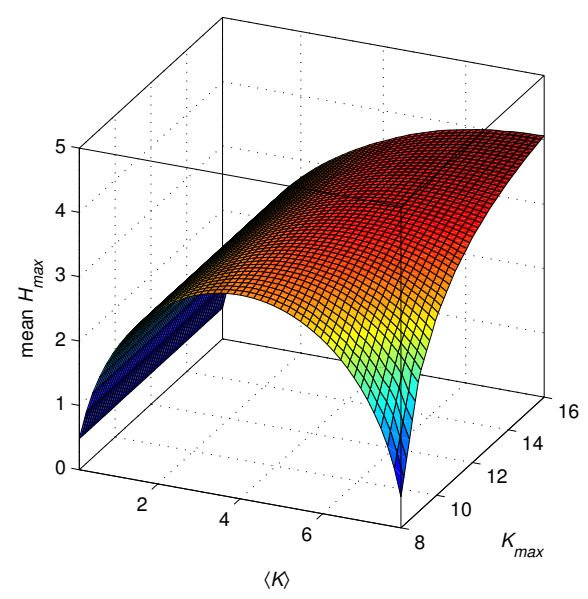

(a) mean of $\mathcal{H}_{\max }$

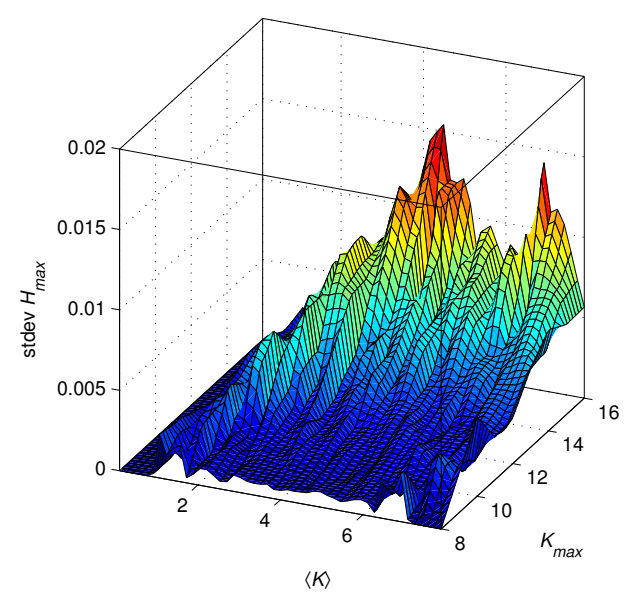

(b) standard deviation of of $\mathcal{H}_{\max }$

Figure 2.11: Mean and standard deviation of the entropy of the truncated exponential distribution $a e^{-b x}$.

maximum at $\langle K\rangle=\frac{K_{\max }}{2}$. For $K_{\max }>10$, the entropy value only increases as $\langle K\rangle$ increases. We also observe that most heterogeneous in-degree distributions happen when $\langle K\rangle=\frac{K_{\max }}{2}$, which coincides with the values for which $a$ is most predictable. We see however that as $\langle K\rangle$ and $K_{\max }$ increase, the value of the entropy shows more fluctuations in the calculated mean (Figure 2.11(b)). For our experiments in the rest of this thesis, we use $K_{\max }=30$ for LSM and $K_{\max }=6$ for RBN. However, the findings about the entropy of the heterogeneous systems can tell us how reliable our results will be. This is important since we will be presenting the dynamical measures of the network as a function of $\mathcal{H}\left(P_{\psi}\right)$.

\subsubsection{Discussion}

In the context of complex systems study, heterogeneity in networks introduces analytical intractability in the study of systems level behavior. This intractability pushes the rigorous study of heterogeneous networks into the realm simulation. The study of complex networks through simulations has the advantage that we 
can finely control the parameters of interest.

We introduced three dimensions of the heterogeneity in complex networks and proposed entropy as a unified measure of heterogeneity. The three dimensions of the heterogeneity are: 1- the in-degree distribution, 2- the functions of the nodes, and 3- locality of the interconnect. We identified the two limiting case of indegree distribution on the heterogeneity axis: uniform and exponential in-degree distribution.

We proposed a simple mutation schema that can evolve a uniform in-degree distribution into an exponential form. Furthermore, we justified why the exponential in-degree distribution is a viable choice for the limiting case of a maximally heterogeneous in-degree distribution. It is possible in a directed evolution that the heterogeneity of the in-degree distribution increases from zero for the uniform distribution to a distribution with an arbitrary shape. However, in the context of self-assembly of a system in a noisy environment, the evolution of the in-degree distribution is dictated by the principles of equilibrium statistical physics and the most likely distribution for systems in the equilibrium. This implies that the only reasonable way to systematically study the heterogeneity in the in-degree distribution is to start from a uniform distribution and to evolve the distribution randomly, in which case the average final in-degree distribution after a sufficiently large number of evolution steps is an exponential in-degree distribution. This exponential in-degree distribution falls under the category of truncated exponential distribution. We formalized our definition of the truncated exponential in-degree distribution and systematically studied the properties of this distribution as the system undergoes natural time evolution.

\subsection{FUNCTION HETEROGENEITY}

In this section we describe the function heterogeneity in RBN and LSM. Despite the difference in the nature of functions in the RBN and LSM, we will see how entropy 
allows us to have a unified vocabulary to talk about function heterogeneity in the network. We will lay out the framework using which we will study the dynamics of RBN and LSM as a function of heterogeneity of their node functions.

In RBN, node functions are Boolean look-up tables randomly. For each node $i$, the function is chosen from the available functions to the node based on the number of input connections of the node $K_{i}$. To study the function heterogeneity in a meaningful way, we only consider homogeneous in-degree RBNs, in which the set of available functions to pick from is the same for all the nodes. We first pick a random function and assign it to all the nodes in the RBN. We will then replace the function of nodes randomly with the other functions picked from the set of available functions. Each time we repeat the replacement the entropy, and therefore the heterogeneity, of functions in the network increases. However, we have to make sure that the new functions have the same bias $p$ as other functions in the network. This is because the average $p$ influences the dynamics, and we would like to control for these effects. We measure the entropy of the functions on the network by calculating the propability of occurance of each function.

In a LSM network with hyperbolic tangent transfer functions [24], one can extend the definition of function heterogeneity in two ways:

1. Each node can have different hyperbolic tangents, e.g., with different slopes.

2. The slopes of hyperbolic tangents are distributed around a mean according to a probability density function.

So far, all the research in the LSM community has been dedicated to networks in which all the nodes have the same transfer function, e.g., hyperbolic tangent (see [24] and references therein). The equation of the hyperbolic tangent function is of the following general form:

$$
\tanh (a x)=\frac{e^{2 \alpha x}-1}{e^{2 \alpha x}+1}
$$




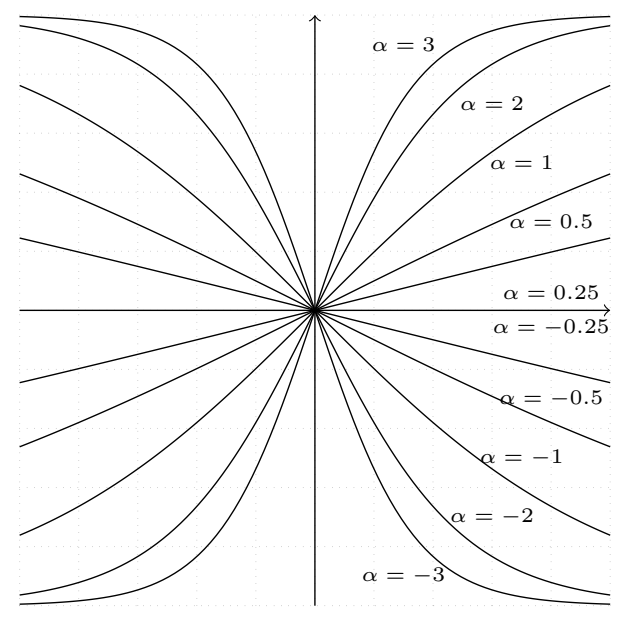

Figure 2.12: Hyperbolic tangent for different values of parameter $\alpha$. The general form of the equation for hyperbolic tangent is $\tanh (a x)=\frac{e^{2 \alpha x}-1}{e^{2 \alpha x}+1}$. We model the heterogeneity as variations in the parameter $\alpha$. The plot illustrates the hyperbolic tangents for $\alpha \in\{-3,-2,-1,-0.5,-0.25,0.25,0.5,1,2,3\}$.

Motivated by bottom-up self-assembled systems, we can think of LSM networks in which each node has a different function. In a simple case, we can assume that all nodes still have a hyperbolic tangent function, but each function has a different value for the parameter $\alpha$ (Figure 2.12). The dynamics and performance of such a system has not been studied. Moreover, we can introduce the variability in $\alpha$ as a design variation. To do so, we build networks in which the parameter $\alpha$ assumes a distribution around a mean $\alpha_{\mu}$. For instance, $\alpha$ could be distributed according to a normal probability density function with the mean $\mu$ and the variance $\sigma^{2}$ as follows:

$$
\mathcal{N}\left(\mu, \sigma^{2}\right)=\frac{1}{\sqrt{2 \pi \sigma^{2}}} e^{\frac{-(x-\mu)^{2}}{2 \sigma^{2}}} .
$$

Figure 2.13 shows the curves for $\mathcal{N}\left(1, \sigma^{2}\right)$, where $\sigma \in\{0.01,0.1,0.4,0.8,10\}$. As $\sigma \rightarrow \infty$, the distribution becomes more uniform. Since we draw the values of $\alpha$ in $\mathrm{Eq} 2.11$ from this distribution, we have a control parameter that can change the diversity of the hyperbolic tangents in the network. A similar method 


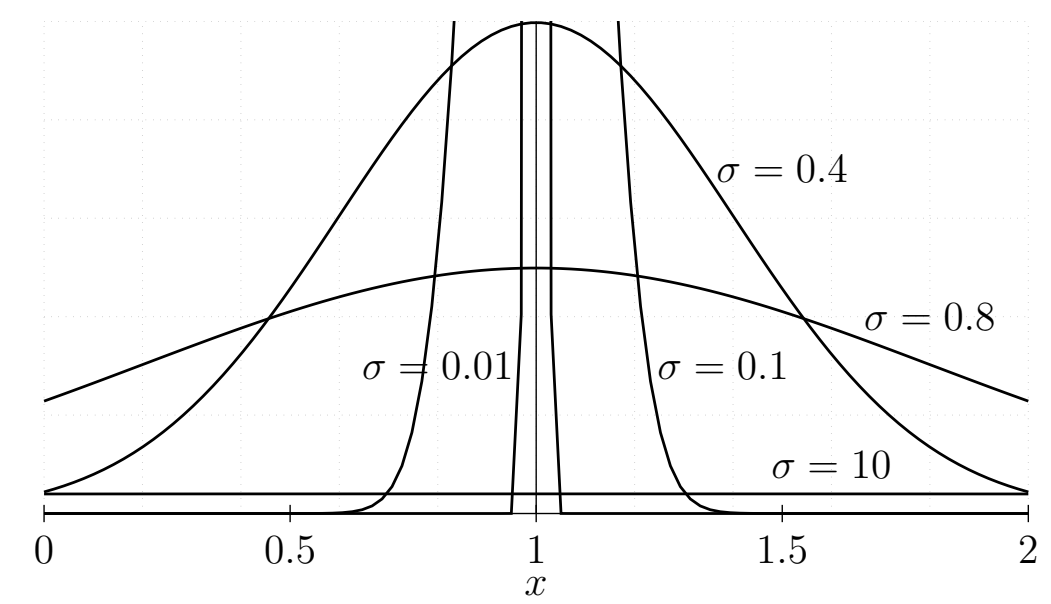

Figure 2.13: Curve of the normal distribution for zero mean $(\mu=1)$ and different standard deviation, $\sigma \in\{0.01,0.1,0.4,0.8,10\}$. The equation for the normal distribution is $\mathcal{N}\left(\mu, \sigma^{2}\right)=\frac{1}{\sqrt{2 \pi \sigma^{2}}} e^{\frac{-(x-\mu)^{2}}{2 \sigma^{2}}}$.

is used in [24] to control the distribution in the weight matrix of the LSM. In order to have a uniform way of characterizing heterogeneity, we continue with the entropy to measure the variability in the distribution. This allows us to use a single heterogeneity parameter to characterize the variability in any distribution. For continuous distributions, however, the entropy is defined as:

$$
\mathcal{H}(\mathbb{X})=-\int_{\mathbb{X}} f(x) \log _{2} f(x) d x
$$

where $f(x)$ is the probability density function and $\mathbb{X}$ is the support of the distribution. Consequently, the entropy of $\mathcal{N}\left(\mu, \sigma^{2}\right)$ in Eq. 2.12 is calculated as:

$$
\mathcal{H}\left(\mathcal{N}\left(\mu, \sigma^{2}\right)\right)=\frac{\log \left(2 \pi e \sigma^{2}\right)}{2}
$$




\subsection{INTERCONNECT IRREGULARITY}

By interconnect irregularity we mean how local or global the connectivity between nodes is. This definition assumes the existence of spatial location of the nodes in a network. The definition of networks in the classical RBN and LSM does not include any space. Here, we redefine the networks used in LSM and RBN and consider spatial embedding for the network. We assume networks that are embedded in a 2-dimensional Euclidean space. First, we arrange the nodes of the network on a 2-dimensional lattice with typical distance $L=1$. The typical distance is the distance between two adjacent nodes along one dimension. We then calculate the distance matrix $D_{M}=r_{i j} . r_{i j}$ is the Euclidean distance between node $i$ and $j$. The probability of a link between node $i$ and $j$ of length $l_{i j}$ will be a function of this distance matrix:

$$
P_{l_{i j}}=F\left(r_{i j}\right)
$$

We will see in Chapter 5 that since we are interested in physical realizability of self-organized networks, we follow a power-law model $F(x)=x^{-a}$ for our link distribution [87]:

$$
P_{l_{i j}}=r_{i j}^{-a}
$$

This model does not conflict with the way we impose a particular in-degree sequence $\psi$ on the network and we are able to combine any in-degree sequence with any interconnect irregularity schema $F(x)$. A similar method was used in [23] to combine different in-degree distribution and spatial embedding schema. Consistent with our other measures of heterogeneity, we continue to use the entropy of the distribution of the wire lengths $\mathcal{H}\left(P_{\mathcal{L}}\right)$, where $\mathcal{L}$ is the sequence of the wire lengths $\left\{l_{i j}\right\}$ in the network. 


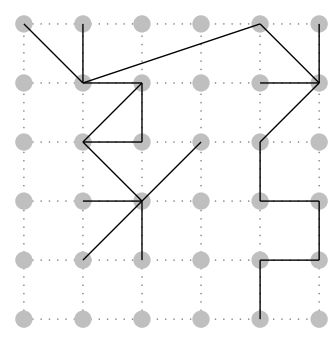

(a) $\alpha=2$

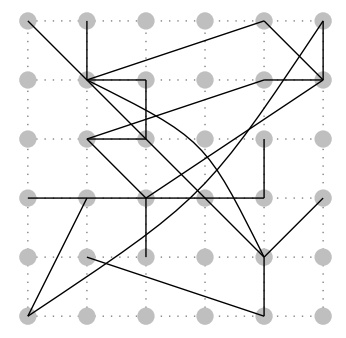

(b) $\alpha=0$

Figure 2.14: Power-law distribution for connection length. The nodes are placed on a mesh with a unit distance between two horizontal and vertical neighbors. (a) Probability of a connection between node $\mathrm{A}$ and node $\mathrm{B}$ with distance $r$ is proportional to $r^{-\alpha}$. (b) For $\alpha>0$, the length distribution will be biased toward short range connections. For $\alpha=0$ the link probability is independent of distance between the source and the destination nodes.

\subsection{MEASURES OF THE NETWORK DYNAMICS}

To study the effects of heterogeneity on the dynamics of networks, we use a number of well-known measures, including: Derrida measure, damage spreading, and kernel quality. Each one of these measures interprets the dynamics of the network differently and measures different aspects of the dynamical regime. We formally introduce these measures and describe what they mean.

\subsubsection{Derrida Measure}

A long standing problem in analyzing the dynamics of incredibly simple systems, such as RBN and CA, is the intractability of the future system state. Derrida made an assumption that after each time step of state update, all the connections on a RBN network will be rewired and all the node functions will be replaced by another randomly chosen function. This assumption makes it possible to ignore dynamical attractors in the RBN and ask the simple question: "At what rate do 
two different states of the system diverge or converge?" The fundamental result of Derrida's work is an equation that calculates the divergence between two states of the network after one update [30]:

$$
d_{t+1}=\frac{1-\left(1-d_{t}\right)^{K}}{2}
$$

Here, the assumption is that all nodes in the network have the same in-degree $K=2$ and the probability of the random Boolean functions on the nodes to output a " 1 " is $p=0.5$. If one changes the bias from $p=0.5$ to a higher or lower value, the system becomes spontaneously ordered. In such systems, higher connectivity is needed to achieve critical dynamics. This sensitivity to $p$ poses challenges to study the function heterogeneity and its effects on the network dynamics. We will restrict ourselves to only limited cases in which we can control the bias and function heterogeneity independently (see Chapter 4). Derrida also proposed that this could be extended to networks with a heterogeneous in-degree distribution as follows:

$$
d_{t+1}=\sum_{K} p(K) \frac{1-\left(1-d_{t}\right)^{K}}{2} .
$$

$p(K)$ is the probability of the node with in-degree $K$. Figure 2.15 shows the analytical and experimental result for Derrida's formula for networks of various in-degree distribution with average connectivity $\langle K\rangle=2$. Sometimes this plot is called Derrida curve. For heterogeneous RBN, the results from the annealed approximation (AA fixed) matches closely with the experimental data (Sim fixed). However, for Poisson graphs, the agreement between prediction (AA Poisson) and experiment (Sim Pos) diverges. The analytical calculation predicts that the curve should be lower than the curve than for homogeneous RBN,i.e., theoretical result predicts that in Poisson RBN the dynamics is more ordered than the homogeneous networks. In contrast, the experimental data shows the curve for the Poisson networks is actually higher than the homogeneous RBNs, which means that the dynamics 


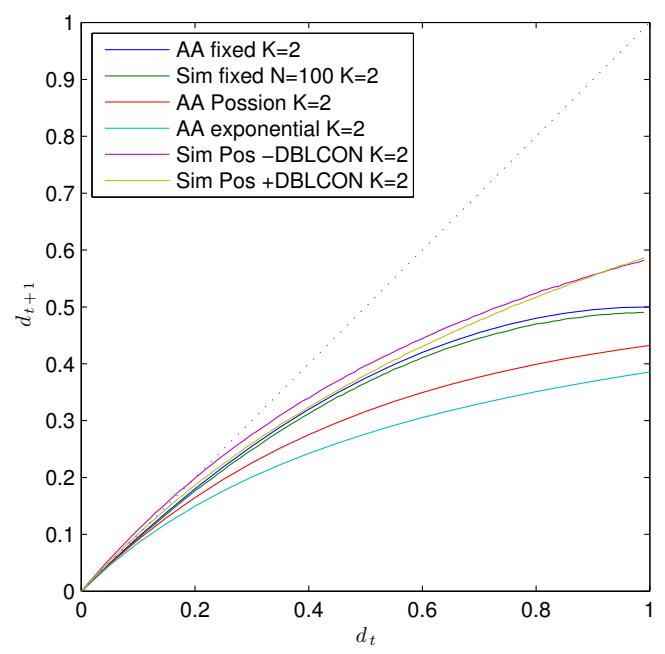

Figure 2.15: Analytical and experimental data for Derrida curve for RBNs with different in-degree distributions.

is richer. For networks with exponential in-degree distribution, the analytical result shows an even lower curve than for Poisson networks meaning the dynamics is predicted to be poorer. Again, the experiment shows that network dynamics for exponential networks is richer than for the Poisson networks. The analytical results qualitatively and quantitatively mispredict the dynamics of the network.

The entropy of the in-degree distribution for the exponential networks is higher than the binomial networks. Therefore, the analytical solution predicts that increasing the heterogeneity in the RBN in-degree will result in poorer dynamics.

In Chapter 3.1 we will see how different in-degree distributions affect the dynamics of Derrida measure and how close the empirical results are to the analytical calculations.

\subsubsection{Damage Spreading}

In section2.6.1 we introduced the Derrida measure for RBN dynamics. Derrida measure predicts how fast a perturbation in a single node spread to other nodes 


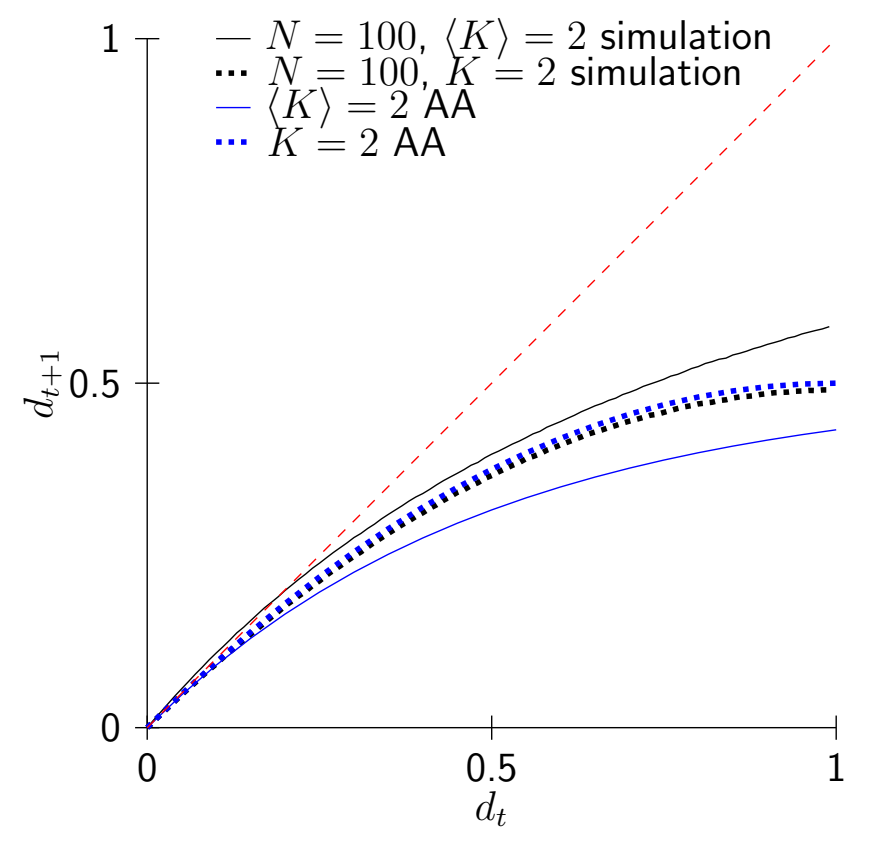

Figure 2.16: Derrida curves for simulated systems and the annealed approximation model. The simulation and theoretical prediction for damage spreading in RBN with exactly $\langle K\rangle=2$ connections per node agree closely. For networks with average connectivity $\langle K\rangle=2$ and a Poisson in-degree distribution, the prediction of the annealed model and the simulation diverge. The annealed model predicts a frozen dynamics for Poisson networks while simulations show the system is chaotic.

in one time step. This measure does not account for limit cycles in the network. To measure how perturbation spreads in a real RBN with limit cycles over a long period of time one could measure damage spreading in RBN. The damage spreading in discrete networks is very similar to the concept of the Lyapunov exponent in continuous dynamical systems. We measure the damage spreading by calculating the divergence between two states that differ only in 1 bit, in the initial state, over long time. To calculate this, we create an RBN and randomly assign an initial state to it. We then create an exact copy of the RBN with the same initial state, but we choose a random node on the duplicate network and flip its state. The 
difference in the initial state of these two RBNs is thus 1 bit. We then run the two networks for a long time $T$ and measure the number of different node states in the final state. This is the Hamming distance between the final state of the two networks. We repeat this measurement over many networks and initial states and calculate the average as follows:

$$
\bar{d}=\frac{1}{z} \frac{1}{c} \sum_{i \in G(N,\langle K\rangle)} \sum_{j \in I C} d_{i, j}(T) .
$$

$d_{i, j}(T)$ is the difference (in bits) between the states of the two copies of the network after $T$ time steps for network $i$ and initial state $j . c$ is the number of initial states $I C$, and $z$ is the number of networks with size $N$ and connectivity $\langle K\rangle$ used for simulations.

\subsubsection{Lyapunov Exponent for LSM}

The Lyapunov exponent $\lambda$ is the classical way of measuring the chaotisity in a dynamical systems [24]. Formally, the Lyapunov exponent is defined as follows [24]:

$$
\lambda=\lim _{t \rightarrow \infty} \frac{1}{t} \ln \left(\frac{H(t)}{H(0)}\right) .
$$

$H(t)$ is the Hamming distance of the two networks at time step $t$. Calculating this value for infinite time is intractable but it has been known that this value can be approximated by calculating the damage spreading for one time step as in [24]. Similarly we calculate $\lambda$ as follows:

$$
\lambda=\ln \left(\frac{H(1)}{H(0)}\right)
$$

where $H(0)=1$. In ordered systems $\lambda<0$ and in chaotic systems $\lambda>0$. Systems with $\lambda=0$ are on the edge between order and chaos and have complex dynamics. For practical reasons, however, in cases where $H(1)=0$, the value of $\lambda=-\infty$. We cannot include these values to calculate averages and therefore we omit these 
cases for $\lambda$ calculations. This causes $\lambda$ for ordered systems to be slightly above 0 . However, this will not affect our results because we will be always comparing the values with $H(1)$ and values of $\lambda$ for chaotic systems.

\subsubsection{Network Topology Measures}

To understand the effects of in-degree heterogeneity and interconnect irregularity on the dynamics of RBN and LSM, one should first be mindful of the way these two aspects of heterogeneity affect the topology of a network. Structure and function of the networks have been the subject of many studies and several measures have been proposed to capture different topological aspects of the networks $[4,20,82]$. A survey of most relevant network measures for complex dynamical networks is compiled by Rubinov and Sporns [94]. Three fundamental network measures characterize functional integration, functional segregation, and modularity in complex networks. We study how these measures change with the connectivity of the network for different in-degree and interconnect heterogeneity. These measures are as follows [94]:

Functional integration. - Characterizes the ability of the network to combine local information from distributed regions of the network. In other words, functional integration determines how easily different parts of the network can communicate. Functional integration can be measured using characteristic path length $L . L$ is defined as the average shortest path length in the network between all pairs of nodes.

Functional segregation. - Refers to the ability of a network to perform specialized processing within highly connected groups of nodes in the network. The existence of functional segregation in the network is indicative of the ability of the network to decompose information processing into functional parts each of which is carried in appropriate regions of the network. The most important measure of functional segregation is local clustering coefficient. Clustering coefficient is the 
fraction of triangles around a single node averaged over all nodes in the network.

Modularity. - Modularity is a more sophisticated segregation measure, which can characterize how cohesive highly connected groups of nodes are in the network. This is quantified by measuring $Q$, which indicates the balance between the links within a group of nodes compared to links in between groups of nodes. This measure indicates how well different functional parts of the network communicate and share information. Completely random networks with no modularity will have $Q=0$ and in completely modular networks $Q=1$. 


\section{IN-DEGREE HETEROGENEITY}

In this chapter, we study the effects of heterogeneity on the dynamics of RBNs and LSMs. We start by measuring the dynamics in the networks using the Derrida measure and the Lyapunov exponent. We then incrementally increase the heterogeneity of the in-degrees in the network and investigate how this heterogeneity affects the dynamics. We will compare our results with existing results in the literature.

\subsection{DYNAMICS OF RANDOM BOOLEAN NETWORKS}

\subsubsection{Derrida Curve and Maximum Entropy In-Degree Distribution}

We begin our study of the critical dynamics in the RBN by calculating the Derrida curves for systems with maximum heterogeneity in their in-degree distribution. To do so, we evolve networks of different average connectivity $\langle K\rangle$ as discussed in Section 2.3.2 to achieve a maximum entropy in-degree distribution.

Figure 3.1 shows the Derrida curves for RBNs with a maximum entropy distribution with average connectivity $\langle K\rangle=1.0,1.5$, and 2.0. We see that in RBNs with maximum entropy distribution, the Derrida curves with $\langle K\rangle=1.0$ and 2.0 seems very similar compared to homogeneous systems (compare with Figure 3.2 and Figure 3.3). The significant difference is that for maximum entropy RBN, the Derrida curve for networks with $\langle K\rangle=2$ lies above the identity line. This means that the $\mathrm{RBN}$ is in the chaotic regime and perturbations in the system spread quickly. These systems are suboptimal for robust adaptive information processing 


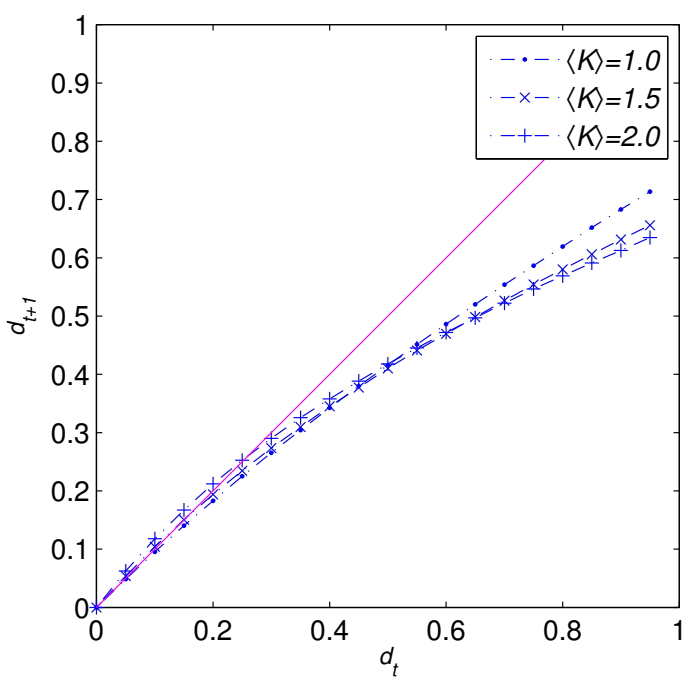

Figure 3.1: Derrida curve for $\langle K\rangle=1.0,1.5$, and 2.0 for RBN with maximum entropy in-degree distribution. We see that both $\langle K\rangle=1.5$ and 2.0 are in the chaotic regime and $\langle K\rangle=1.0$ is in the critical regime.

because any noise in the system spreads in the network and damages the information that the network needs for computation. The Derrida curves for RBNs with average connectivity $\langle K\rangle=2$ are also very close to the identity line but still below. This means that heterogeneous RBNs, even when in the ordered phase, show critical dynamics and are suitable for complex computation.

A natural question to ask is how the heterogeneity changes the Derrida curve for RBNs with different average connectivity $\langle K\rangle$. We answer this question in the next section.

\subsubsection{Change in Derrida Curve}

In our preliminary study (see Section 3.1.1), we saw how the maximum heterogeneity in-degree distribution affects the dynamics of the RBN. Here, we will see how the Derrida curve for the networks of different average connectivity changes 


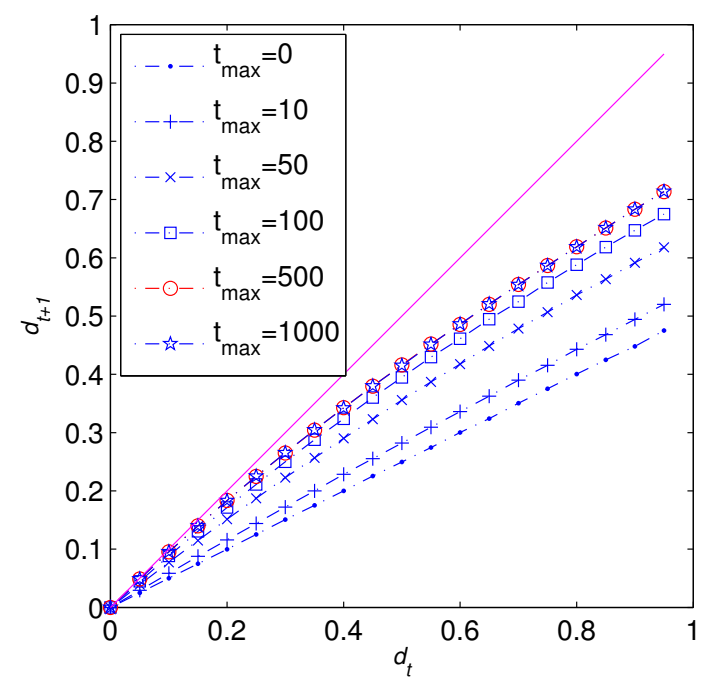

Figure 3.2: The Derrida curve of $\mathrm{RBN}$ with $N=100$ and $\langle K\rangle=1.0$ for different number of evolutionary time steps. Higher time steps result in more heterogeneous in-degrees. The higher the in-degree heterogeneity, the richer the network dynamics. Curves below the identity line are in ordered regime, and curves above the identity line are in chaotic regime. The closer a curve to the identity line is, the closer the dynamics is to the critical dynamics.

as the in-degree distribution evolves from a completely homogeneous state to a maximally heterogeneous one.

To perform this study, we create an initial population of networks with a homogeneous in-degree distribution and evolve their in-degree until we reach the maximum entropy in-degree distribution as discussed before. At each step of the in-degree evolution, we calculate the Derrida curve for the network. Figure 3.2 shows the Derrida curve of a network with 100 nodes and an average connectivity of $\langle K\rangle=1.0$. This plot shows the fraction of perturbed nodes in the network at time step $t+1$, i.e., $d_{t+1}$ as a function of fraction of perturbed nodes at time $t$, i.e., 
$d_{t}$. The curve corresponding to $t_{\max }=0$ shows the Derrida curve for the initial homogeneous network. We see that for higher number of mutations on the in-degree sequence, the Derrida curve shifts higher and higher and gets closer to the identity line, i.e. $d_{t+1}=d_{t}$. This means that the dynamics of the network is getting closer to the critical dynamics. For homogeneous systems, any size of damage at time $t$ will start to fade on the next time step. As the in-degree distribution evolves towards a more heterogeneous distribution, the number of nodes with $K>1$ increases. These nodes accept incoming perturbations from many nodes and redistribute it to the network. This causes the damage $d_{t}$ at any time step to be amplified and thus the system exhibits critical dynamics. The in-degree heterogeneity increases the ability of the networks to redistribute signals more effectively.

Figure 3.3 shows the Derrida curve for a network 100 nodes with average connectivity $\langle K\rangle=2.0$. We see a general trend of upward shift in the Derrida curve as the in-degree distribution evolves toward maximum entropy distribution. However, this change is more subtle at first, but includes a jump somewhere between time steps 100 and 500. After 500 mutations, the changes in the Derrida curve becomes very slight again. Evidently, the rate of change in the Derrida curve as a function of evolution time is also a function of average connectivity in the network.

We take our investigation one step further and study how does the Derrida curve changes as the entropy of in-degree distribution increases. We first have to isolate an essential aspect of the Derrida curve which identifies the critical dynamics in the network, namely $\frac{d_{t+1}}{d_{t}}$. When this quantity is below 1 , the dynamics is in the ordered regime because the perturbations in the systems die out over time. For values above 1, any perturbation in the network spread to all the nodes over time and interfere with the local processes in different clusters of nodes. This is why these networks are not suitable for information processing. For networks in which $\frac{d_{t+1}}{d_{t}}=1$, the perturbations remain the same over time. We calculate this fraction over time as a function of the in-degree distribution in the network for networks 


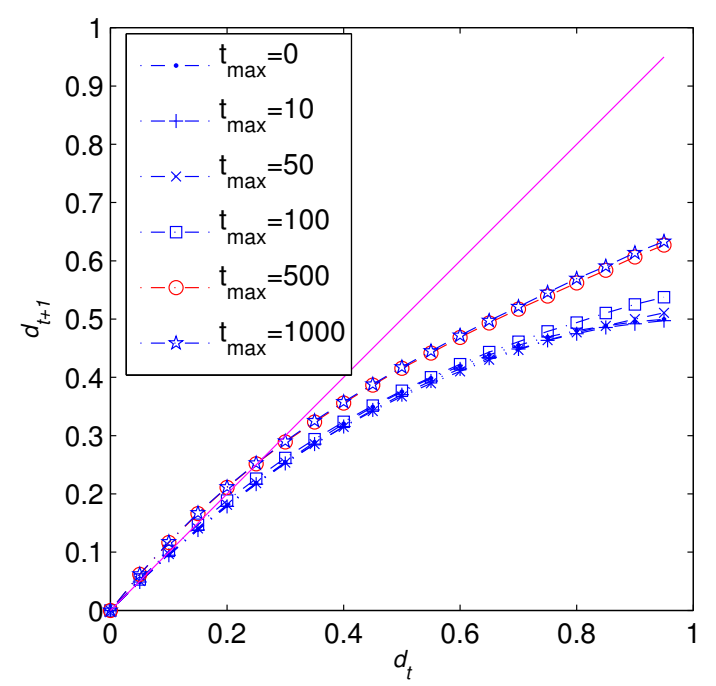

Figure 3.3: The Derrida curve of $\mathrm{RBN}$ with $N=100$ and $\langle K\rangle=2.0$ for different number of evolutionary time steps. Higher time steps result in more heterogeneous in-degrees. The higher the in-degree heterogeneity, the richer the network dynamics. In this case, the system is already in critical regime. The higher heterogeneity pushes the system into the chaotic region. Curves below the identity line are in ordered regime, and curves above the identity line are in chaotic regime. The closer a curve to the identity line is, the closer the dynamics is to the critical dynamics.

with different average connectivity $\langle K\rangle$.

Figure 3.4 shows the $\frac{d_{t+1}}{d_{t}}$ as a function of time throughout the evolution of the in-degree distribution in the networks for network of average connectivity $\langle K\rangle=$ 1.0,2.0, and 3.0. For networks with average connectivity $\langle K\rangle=1.0$, the fraction increases rapidly at first and then levels right below the $\frac{d_{t+1}}{d_{t}}=1$ with no change. For $\langle K\rangle=2.0$, the fraction $\frac{d_{t+1}}{d_{t}}$ goes over the values 1 after only $t=50$ evolution time steps in the in-degree distribution and reaches a stationary level near $t=$ 500. For $\langle K\rangle=3.0$, the value of the fraction starts from above 1.4 and after a little increase reaches the stationary value of the $\frac{d_{t+1}}{d_{t}}=1.58$ at about $t=500$ 


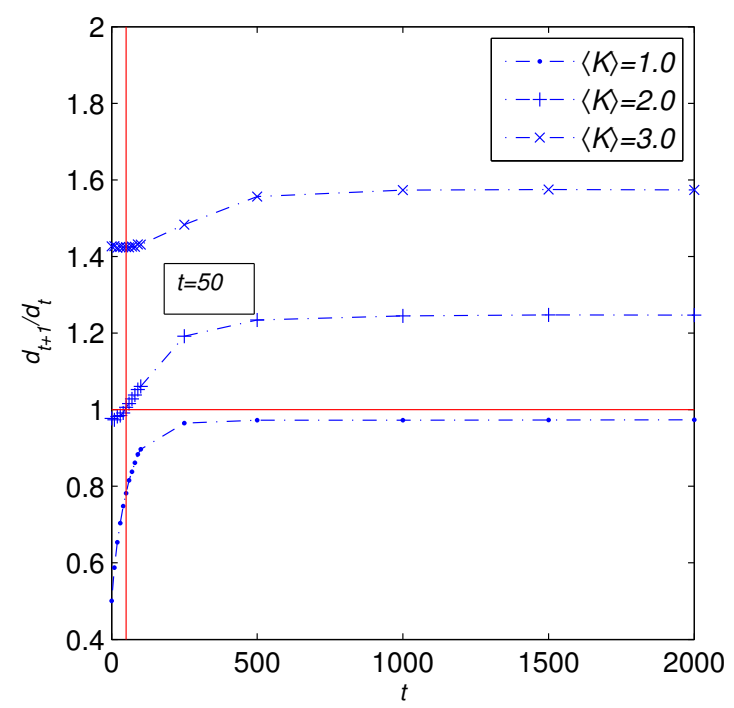

Figure 3.4: The change in $\frac{d_{t+1}}{d_{t}}$ as a function of numbers of time steps. The closer a system is to $\frac{d_{t+1}}{d_{t}}=1$ the richer the dynamics. We observe that high heterogeneity in $\mathrm{RBN}$ makes the dynamics of ordinarily ordered systems, i.e., $\langle K\rangle=1.0$, richer while pushing the dynamics of critical systems, i.e., $\langle K\rangle=2.0$, into the chaotic regime.

evolutionary time steps. According to this data, the higher the average connectivity the less significant the change in the $\frac{d_{t+1}}{d_{t}}$ over time. Also, the most significant change occur for $\langle K\rangle=1.0$, where the normally ordered system gets closer to the critical dynamics and $\langle K\rangle=2.0$ where the normally critical network shows chaotic behavior after a few evolutions in the in-degree distribution. Figure 3.4 shows very clearly how the dynamics change as the in-degree distribution becomes heterogeneous and at what rate this change happens. According to this plot, the sparser the network, the more sensitive it is to changes in the heterogeneity in the in-degree distribution.

Next, we directly measure the fraction $\frac{d_{t+1}}{d_{t}}$ as a function of entropy of the indegree distribution in the network. Figure 3.5 shows the result of this measurement 


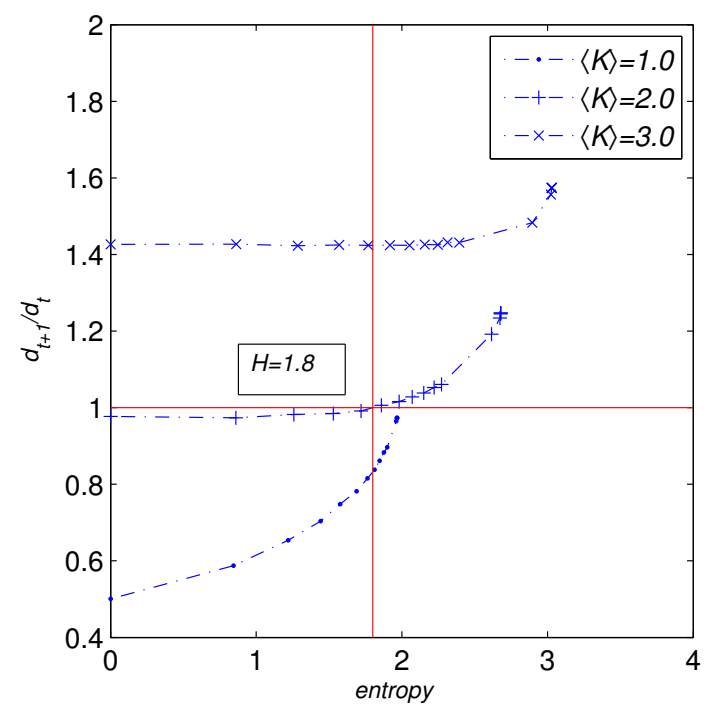

Figure 3.5: The change in $\frac{d_{t+1}}{d_{t}}$ as a function of entropy of in-degree distribution. The closer a system is to $\frac{d_{t+1}}{d_{t}}=1$ the richer the dynamics. We observe that high heterogeneity in RBN makes the dynamics of ordinarily ordered systems, i.e., $\langle K\rangle=1.0$, richer while pushing the dynamics of critical systems, i.e., $\langle K\rangle=2.0$, into the chaotic regime.

for systems of $N=100$ nodes and average connectivity $\langle K\rangle=1.0,2.0$, and 3.0. We see that for $\langle K\rangle=1.0$, the ratio $\frac{d_{t+1}}{d_{t}}$ starts increasing. The rate of this increase speeds up as the entropy of the in-degree indstribution $\mathcal{H}\left(P_{\psi}\right)$ increases. This increase finally stops right below the critical dynamics $\frac{d_{t+1}}{d_{t}}=1$. For RBNs with $\langle K\rangle=2.0$ the picture his quite different. At first there is no significant change in $\frac{d_{t+1}}{d_{t}}$. Near $\mathcal{H}\left(P_{\psi}\right)=1.8, \frac{d_{t+1}}{d_{t}}$ approaches 1 , at which point the becomes chaotic for $\frac{d_{t+1}}{d_{t}}>1.8$. The increase in $\frac{d_{t+1}}{d_{t}}$ speeds up after the system enters the chaotic phase and stops maximum entropy value. Finally for $\langle K\rangle=3.0$, the system shows constant $\frac{d_{t+1}}{d_{t}}$ for higher values of $\mathcal{H}\left(P_{\psi}\right)$. For $\mathcal{H}\left(P_{\psi}\right)>2.5$ the fraction $\frac{d_{t+1}}{d_{t}}$ shows a sudden increase and stops when the in-degree distribution reaches the maximum entropy. 
These findings might at first be puzzling. However, we showed previously (Figure 3.4) how the entropy production rate changes in the system under evolution. The entropy of the in-degree distribution shows a very rapid increase at first and quickly reaches the stationary value that corresponds to the entropy of the equilibrium distribution. We take these findings one step further to find the optimal connectivity for the maximally heterogeneous systems for which $\frac{d_{t+1}}{d_{t}}=1$.

According to these results, systems with $\langle K\rangle=2.0$ and homogeneous in-degrees are actually subcritical. With the increased heterogeneity in the in-degrees the system becomes more and more critical. These systems become chaotic for values of $\mathcal{H}\left(P_{\psi}\right)>1.8$. For ordered systems with $\langle K\rangle=1.0$ with homogeneous in-degrees the dynamics is completely ordered until the system reaches the maximum entropy in the in-degree, at which point due to effective signal transfer in the system, the network dynamics become critical. Therefore, systems that are traditionally thought of as ordered can actually show critical behavior if one introduces heterogeneity in the in-degree distribution of the system.

\subsubsection{Optimal Connectivity in Heterogeneous RBN}

In this section, we study how heterogeneity affects the critical connectivity $\langle K\rangle=$ $K_{c}$ of the network, i.e., we find an optimal connectivity for which $\frac{d_{t+1}}{d_{t}}=1$. The perturbations in these systems do not die out or spread and therefore they are optimal for information processing. We start with populations of the networks with average connectivity $1.00<\langle K\rangle<1.30$ with 0.01 increments. We evolve the indegree sequence of each network in the population until it reaches the equilibrium

in-degree distribution and then we calculate $\frac{d_{t+1}}{d_{t}}$. Figure 3.6 shows the value of $\frac{d_{t+1}}{d_{t}}$ as a function of $\langle K\rangle$. We find that for $K_{c}=1.157$, the ratio $\frac{d_{t+1}}{d_{t}}=1$, which means that the system is at the edge of chaos. This is the value of critical connectivity for RBN with maximum entropy in-degree distribution. This values is very close to 1.0. If one defines the cost of building such a system by the average number of 


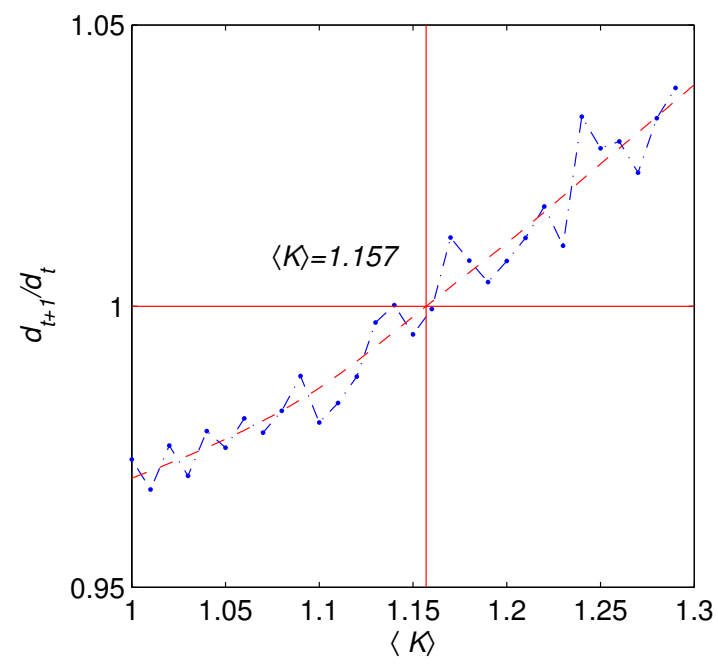

Figure 3.6: Critical connectivity $\langle K\rangle=K_{c}=1.157$ for which $\frac{d_{t+1}}{d_{t}}=1$ for RBNs with maximum entropy in-degree distribution. For fully heterogeneous RBN the critical connectivity is much lower than that of homogeneous systems, i.e., $K_{c}=2.0$.

connections per node, one would reduce the cost of building a network with critical dynamics by increasing heterogeneity in the in-degree distribution of the network.

\subsubsection{Damage Spreading and Heterogeneity}

Rohlf et al. [92] investigated the damage spreading (see Section 2.6.2) in Boolean and threshold networks at the sparse percolation limit. They found that there exists a critical average connectivity $\langle K\rangle=K_{s}$ at which damage spreading in the network becomes independent of the system size $N$. Lu and Teuscher [73] extended this result to spatial and small-world networks. We further investigate the effects of the damage spreading in networks with heterogeneous in-degree distribution. The significance of this regime is in scalability of these systems. The information processing in these systems is more reliable and predictable. Such systems 


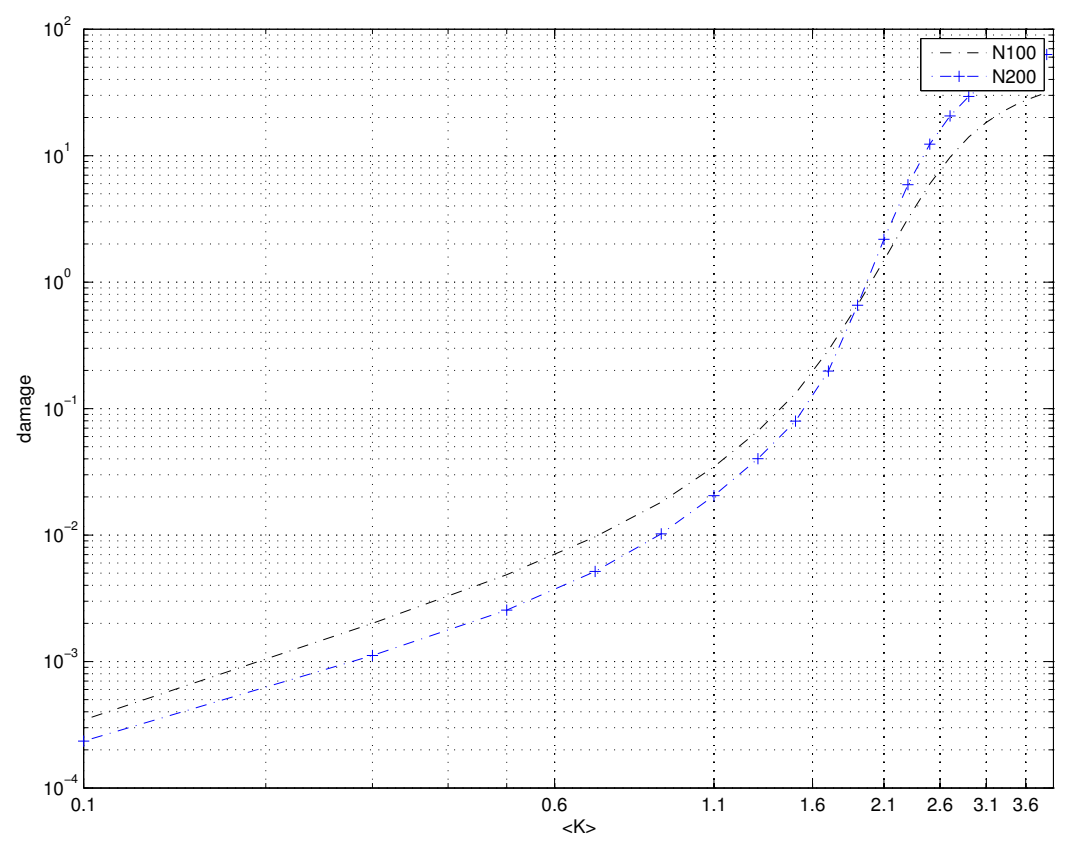

Figure 3.7: Damage spreading in $\mathrm{RBN}$ of $N=100$ and $N=200$. The in-degree distribution is Poissonian. We see that information processing and noise dampening in Poisson networks is at the connectivity $\langle K\rangle \approx 1.8$. This connectivity balances the communication and resilience of the network.

maximize the information transfer in the network while minimizing the effects of unnecessary perturbation, such as noise.

Figure 3.7 shows the result of these simulations for RBNs of size $N=100$ and $N=200$. Here, $T=100, c=100$, and $z=100$. The resulting $\bar{d}$ is averaged over 100 runs.

The study of damage spreading is important to understand the scalability of information transmission and noise propagation in network systems. We can extend this study to heterogeneous networks with different in-degree distribution and study the change in the value of $K_{s}$. In addition, we can investigate the value of the $K_{s}$ as a function of $\mathcal{H}$ for a fixed system size. In previous sections we observed that 
as the in-degree distribution moves from a homogeneous form to a heterogeneous form, the critical connectivity $K_{c}$ that separates the ordered and chaotic regimes goes below 2. This Derrida measurement is in a sense the rate at which two nearby states diverge. Therefore, we expect that the divergence of the damage in the long run will be also affected by this rate. Here, we study the damage spreading in homogeneous networks, Poissonian networks, and exponential networks. Figure 3.8 shows the damage spreading of these networks for $N=100$ and $N=200$ as a function of $\langle K\rangle$. We find that as the heterogeneity in the in-degree distribution increases, the value of $K_{s}$ decreases slightly. However, we cannot easily detect this effect. Therefore, over long run for static inputs, the effect of heterogeneity in the damage spreading of the network is negligible.

\subsection{DYNAMICS OF LIQUID STATE MACHINE}

Now we extend our study of network dynamics to Liquid State Machine, (LSM). We use two different measures to study the dynamics of the LSM for networks with heterogeneous in-degree distribution:

1. The damage spreading rate $H$.

2. The Lyapunov exponent $\lambda$.

\subsubsection{Lyapunov Exponent and In-degree Heterogeneity}

In this section we study the damage spreading and Lyapunov exponent of LSMs with heterogeneous in-degree distributions. To create heterogeneous in-degree networks we start from a homogeneous in-degree networks and we mutate for many time steps $t$ as described in Chapter 2. For each mutation in the in-degree we calculate the damage spreading $H$ and the Lyapunov exponent $\lambda$ as described in Chapter 2. We average the results over 1,000 networks and initial states. The error 


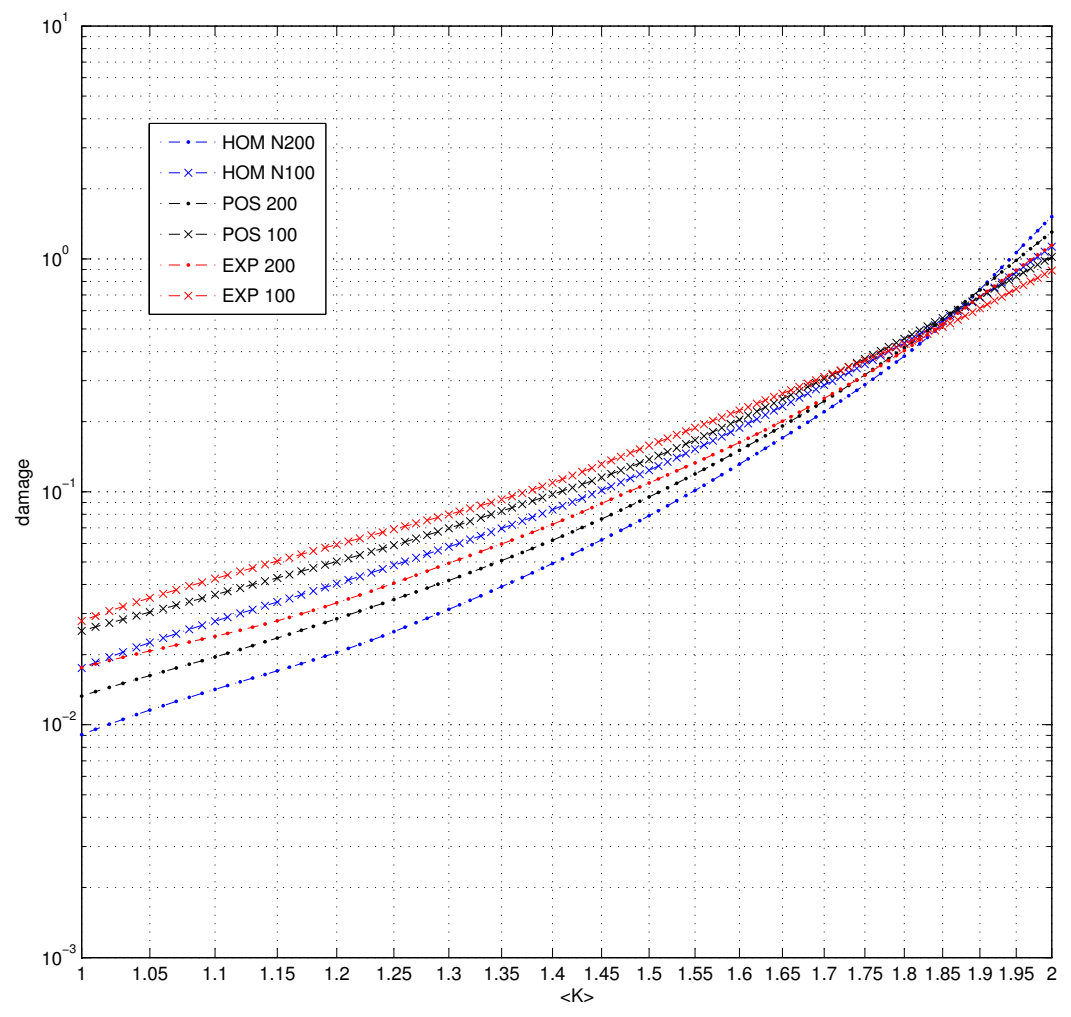

Figure 3.8: Damage spreading in homogeneous networks, Poissonian networks, and exponential networks for $N=100$ and $N=200$. Over long run for static inputs, the effect of heterogeneity in the damage spreading of the network is negligible.

bars are calculated using the standard error: $S E=\frac{\sigma}{\sqrt{n}}$, where $\sigma$ is the standard deviation of the sample and $n$ is the sample size.

$H$ and $\lambda$ are both measures of dynamics in dynamical systems. Systems with $H<1$ or $\lambda<0$ are in the ordered regime while for $H>1$ and $\lambda>0$ the systems are in the chaotic regime.

Figure 3.9 shows the rate of spreading of a smallest admissible damage, i.e. 1 bit, in the state of the LSM in one time step for a network of $N=100$ nodes and average connectivity $\langle K\rangle=1$ (blue), 2 (red), 5 (black), 10 (green). We observe 


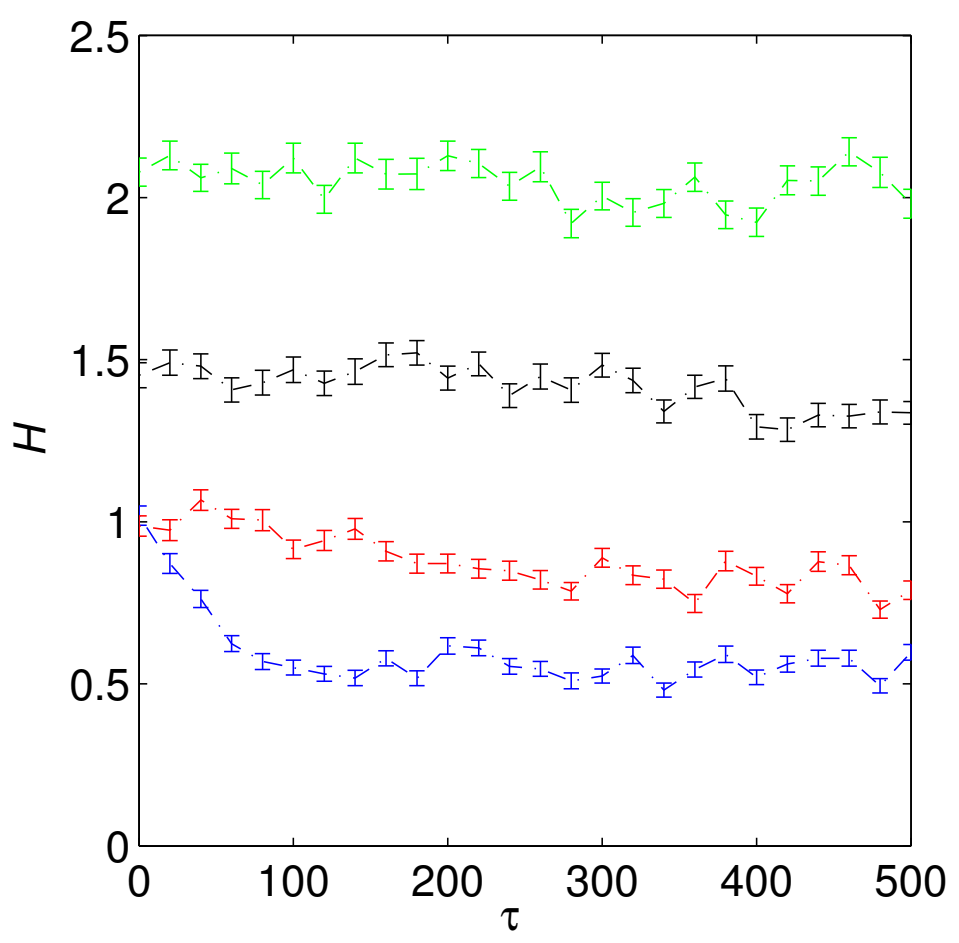

Figure 3.9: Damage spreading in networks with heterogeneous in-degree distribution as a function of number mutation in the in-degree distribution $t .\langle K\rangle=1.0$ (blue), $\langle K\rangle=2.0$ (red), $\langle K\rangle=3.0$ (black), $\langle K\rangle=4.0$ (green). High in-degree heterogeneity result in poorer dynamics in LSM.

for homogeneous networks, i.e. $t=0$, the spreading rate $H=0$ is the same for networks of $\langle K\rangle=1$ and $\langle K\rangle=2$. By $t=100$ time steps, $H$ for networks of connectivity $\langle K\rangle=1$ drops to $H=0.5$ and remains constant. For $\langle K\rangle=2$ the $H=0$ for $t=0$ and over 500 time steps of mutations the $H$ slowly decreases to $H \approx 0.75$. For $\langle K\rangle=5$ and $10 H$ is nearly constant for all time steps $t$. Figure 3.10 shows the Lyapunov exponent of heterogeneous networks of connectivity $\langle K\rangle=$ 1,2,5, and 10. The same behavior we observed for $H$ appears in the Lyapunov exponent but in a quantitatively different way. One strange aspect of Figure 3.10 is that for the ordered regime, where $H<1$, the Lyapunov exponent becomes 
$\lambda>0$. As we explained before, $\lambda$ is the logarithm of the rate at which damage propagates in the system. Therefore, ordered system, must have $\lambda=0$. However, we see that for all heterogeneity levels, $\lambda>0$, even for systems with average connectivity $\langle K\rangle=1$. This is because the $\lambda$ calculation depends on $H$ as follows (see Chapter 2):

$$
\lambda=\ln (H)
$$

If $H=0$ then $\ln (H) \rightarrow \infty$, and we are unable to average over the value of $\lambda$. In our simulations we omitted all instances in which $\lambda \rightarrow \infty$. This causes the average to show slightly higher values for ordered systems.

For highly connected networks, i.e., $\langle K\rangle>2$, the damage spreading is $H>1$, which implies that the system is in the chaotic regime and suboptimal for complex computation. At this regime, damage spreading is independent of the number of mutations in the in-degree distribution and the increase of entropy and therefore the heterogeneity in the in-degree distribution. For $\langle K\rangle=1$ and 2 , we see that the damage spreading $H$ and the Lyapunov exponent $\lambda$ decreases with increased heterogeneity in the network. This decrease is more pronounced for $\langle K\rangle=1$ with a sudden drop from $t<100$ and then a constant value for $t>100$. At this connectivity level the homogeneous LSM shows complex dynamics and as the indegree distribution becomes more and more heterogeneous the system goes into the ordered regime.

\subsection{CONCLUSION}

We studied the network dynamics in LSM and RBN using the Derrida measure and the Lyapunov exponent. We observed for $\mathrm{RBN}$, the higher heterogeneity in the system result in richer dynamics. RBNs that are in ordered regime, i.e. $\langle K\rangle<2$, will have a critical dynamics using maximally heterogeneous in-degree distribution. RBNs that have critical dynamics using homogeneous in-degree distribution will 


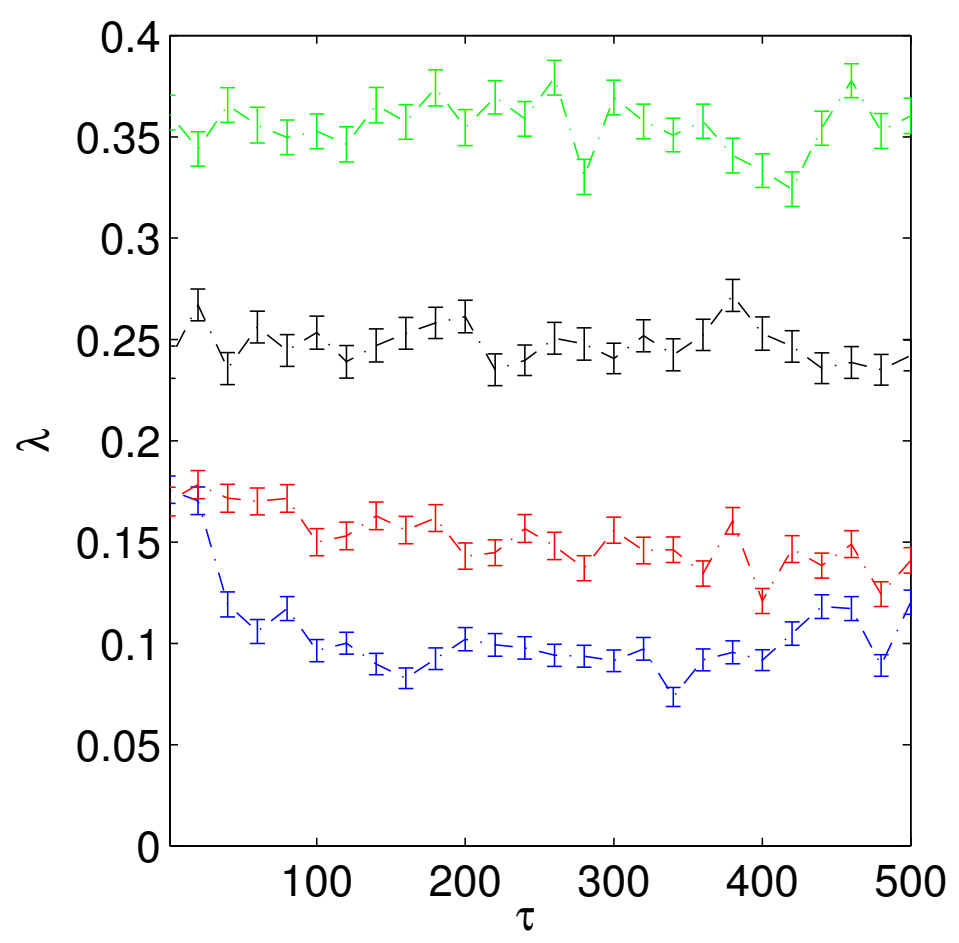

Figure 3.10: Lyapunov exponent in networks with heterogeneous in-degree distribution as a function of number mutation in the in-degree distribution $t .\langle K\rangle=1.0$ (blue), $\langle K\rangle=2.0$ (red), $\langle K\rangle=3.0$ (black), $\langle K\rangle=4.0$ (green). High in-degree heterogeneity result in poorer dynamics in LSM.

be pushed to the chaotic regime with heterogeneous in-degree distribution. These result agree with the results previously reported in [96].

For LSM on the other hand, the higher heterogeneity in the in-degree distribution will result in poorer dynamics since the systems will be pushed into the ordered regime. Similar results has been observed in [64], where the more heterogeneous in-degrees result in lower dynamical range on network of coupled oscillators. 


\section{FUNCTION HETEROGENEITY}

\subsection{FUNCTION HETEROGENEITY IN RANDOM BOOLEAN NET- WORK}

In this chapter, we study the effects of function heterogeneity on RBN dynamics. We use the Derrida measure to calculate how it speeds up or slows down the divergence of two close states. RBN dynamics are heavily influenced by the average connectivity in the network $\langle K\rangle$ and the bias $p . p$ is the fraction of " $1 \mathrm{~s}$ " in the look-up table of the functions averaged over all the nodes in the network. Many analytical and experimental works have studied how $\langle K\rangle$ and $p$ affects the dynamics of RBN $[5,6,30,51,80,88,100]$. This body of work establishes that bias $p$, and a closely related concept of canalization, cause stability in the dynamics of RBN. Canalization refers to situations when the output of a Boolean function is determined completely by the value of one of its inputs. There is an indirect relation between $p$ and canalization in which the higher $p$, the more canalizing the functions are, and thus the network dynamics become more stable. This connection between $p$ and the dynamics poses a serious challenge in studying the effects of function heterogeneity on network dynamics. Additionally, in networks with heterogeneous in-degree distribution, where each node with a different number of inputs $K$ may only accept $2^{2^{K}}$ functions, introduces more difficulty in consistently controlling $p$. Therefore, we need to restrict ourselves to be able to easily control for the type of node functions.

To study RBN dynamics as a function of heterogeneity we must control for 


\begin{tabular}{cc|c}
$\mathrm{A}$ & $\mathrm{B}$ & $\mathrm{F}$ \\
\hline 0 & 0 & 1 \\
0 & 1 & 1 \\
1 & 0 & 1 \\
1 & 1 & 0
\end{tabular}

Figure 4.1: NAND function. The decimal value of the function is $0 \times 2^{3}+1 \times 2^{2}+$ $1 \times 2^{1}+1 \times 2^{0}=7$.

the bias $p$. We narrow this study to $\langle K\rangle=2$ for networks with homogeneous in-degree distribution. There are $2^{2^{2}}=16$ functions of five possible values of $p=0,0.25,0.5,75,1$. We typically call the functions by the decimal value of the output column in the look-up table function, where the first line of the look-up table is the least significant bit and the last line is the most significant bit [114]. Figure 4.1 shows the NAND function of two input bits $A$ and $B$. The decimal value of the function is $0 \times 2^{3}+1 \times 2^{2}+1 \times 2^{1}+1 \times 2^{0}=7$. The bias of NAND function is $p=0.75$. There are 4 different functions with this bias: 7 (NAND), 11 , 13, and 14 (OR). Homogeneous RBN with NAND functions are of interest because of universality of NAND function. Networks of NAND have been proposed before by Turing and studied by Teuscher [107].

Here we study the Derrida measure of the RBN networks with homogeneous functions $7,11,13$, and 14 . We picked these functions because they all have identical bias, i.e., $p=0.75$. For each class of networks we gradually add the other three types of functions uniformly to increase the heterogeneity and measure the dynamics. For example, for networks with homogeneous functions of NAND we replace three node functions by functions 11, 13 , and 14. In a network of 100 nodes, we can repeat this process 25 times to achieve a heterogeneous network in which there are 25 nodes with each function 7, 11, 13, and 14. Note that during this process, the bias $p$ of the network remains constant while the entropy of the 


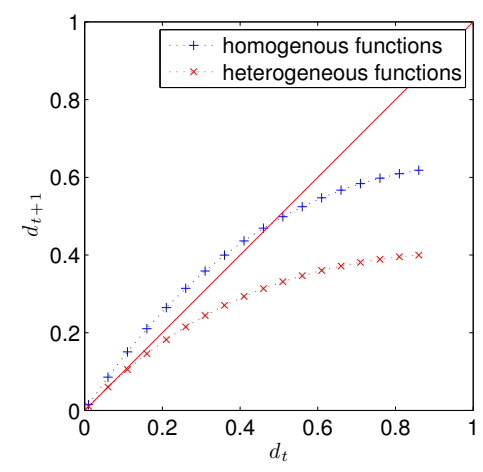

(a) Func 7 (NAND)

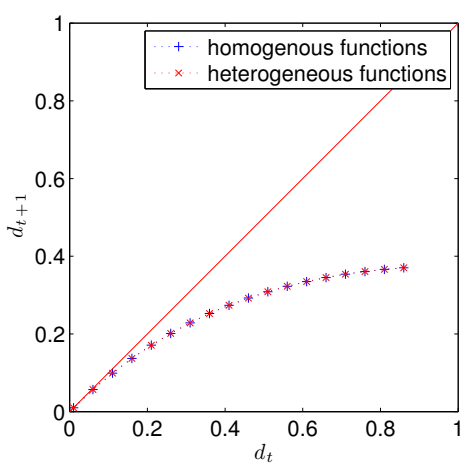

(c) Func 13

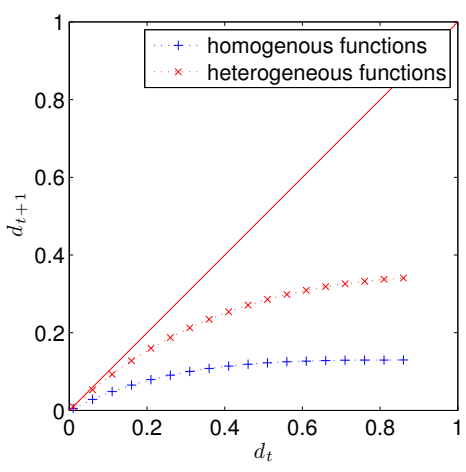

(b) Func $14(\mathrm{OR})$

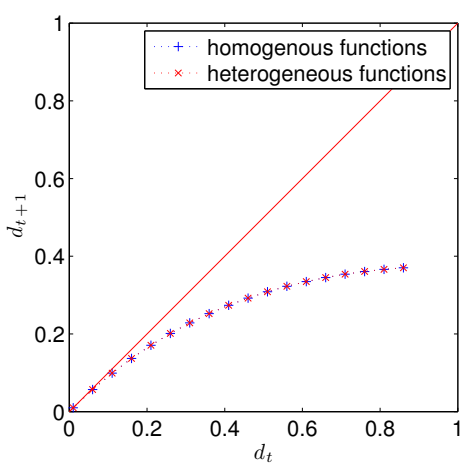

(d) Func 11

Figure 4.2: Derrida curves for RBN networks of uniform in-degree with $K=2$ and bias $p=0.75$ using network functions that are homogeneous, i.e., $\mathcal{H}\left(P_{\mathcal{F}}\right)=0$, and heterogeneous, i.e., $\mathcal{H}\left(P_{\mathcal{F}}\right)=2.05$.

function distribution $\mathcal{H}\left(P_{\mathcal{F}}\right)$ increases from 0 to 2.05 bits, which is the entropy of the maximally heterogeneous function distribution with fixed $p=0.75$. We average the result over 1,000 runs.

Figure 4.2 shows the Derrida curves for networks with homogeneous (blue) and heterogeneous (red) functions. We know from the properties of the Derrida curve that if the curve lies above the identity line, the system is chaotic. The curves below the identity line correspond to ordered systems and curves that are tangent to the identity line correspond to critical systems at the edge of chaos. The 
perturbation in the critical systems will not die out or spread out, and therefore these systems are said to be optimal for information processing. Derrida's annealed approximation (see Chapter 2) predicts that for systems with $K=2$ and $p>0.5$, the dynamics should be more stable and therefore the Derrida curve for these systems should lie below the identity line. The annealed approximation does not assume any particular distribution of the functions in the network as long as the functions on average have a given bias $p$. However, here we see that in RBNs with homogeneous functions, depending on the type of functions on the nodes, we have three different regimes: chaotic for function 7 , ordered for function 14 , and critical for functions 11 and 13. In all cases, as the heterogeneity in the functions increases, the dynamics of the network approaches the critical regime and at the maximum heterogeneity, i.e., $\mathcal{H}\left(P_{\mathcal{F}}\right)=2.05$, the dynamics becomes critical. There is a paradox between this result with the prediction of the annealed approximation: that is, the system is critical according to the experiment. However, the bias of the system is $p=0.75$, which means at connectivity $\langle K\rangle=2$, it should be in the ordered phase. However, the heterogeneity seem to reduce the three different possible to just a single critical phases and therefore the system is more predictable with increased function heterogeneity.

We repeat this experiment with $\mathrm{RBNs}$ with $\langle K\rangle=2$ with homogeneous indegree distributions, and homogeneous functions with bias $p=0.5$. These systems are of interest because networks with $\langle K\rangle=2$ and $p=0.5$ are predicted to be critical, regardless of the distribution of the functions. There are 6 Boolean functions with $p=0.5$ with two inputs: $3,5,6,9,10$, and 12 . To create heterogeneous functions on these networks we start from network with functions that are uniformly one of the listed functions with $p=0.5$ and we repeatedly replace the node functions with other functions not present in the network for maximum of 16 times. This will result in a network with the identical bias $p=0.5$ and a maximally heterogeneous functions with $\mathcal{H}\left(P_{\mathcal{F}}\right)=4.96$. 
Figure 4.3 shows the Derrida curve for homogeneous function RBNs with the named functions and their heterogeneous counterparts. We see that for functions $3,5,10$, and 12 , the dynamics of homogeneous system lies completely on the identity line while the heterogeneous networks show slightly chaotic dynamics. For functions $6(\mathrm{XOR})$ and $9(\overline{\mathrm{XOR}})$, both heterogeneous and homogeneous systems are completely chaotic. In these two types of networks, the number of original functions 6 and 9 is 4 units larger than the other functions when the network approaches maximum function heterogeneity. However, we see that this slightly higher number of functions 6 and 9 actually dominate the dynamics of the network and therefore the heterogeneous networks do not show much difference in the dynamics from the homogeneous networks. This means that for $p=0.5$, the dynamics are very sensitive to the type of the functions and not just the bias $p=0.5$.

Considering the 10 different homogeneous networks that we observed, we conclude that the dynamics of RBN are heavily influenced by the exact type of the functions present in the networks. Some functions such as XOR and $\overline{\mathrm{XOR}}$ completely influence the dynamics of the networks even if their number is slightly higher than the other functions in the network. These findings show that although the annealed approximation does not depend on the exact function to predict the dynamics of the network, the type of functions present in the network influence the dynamics heavily. Without the presence of all the available functions in the network, the dynamics will be unpredictable. Therefore, from the point of view of self-assembled systems, it is absolutely crucial that the system contains all variety of the functions to have a reliable dynamics. Function heterogeneity is therefore beneficial for self-assembled RBNs.

\subsection{FUNCTION HETEROGENEITY IN LIQUID STATE MACHINES}

In this section we show how the heterogeneity in the functions of the LSM affect the dynamics of the system using the damage spreading measure $H$, the Lyapunov 
exponent $\lambda$, and the generalization rank. Normally, all LSMs are built using a nonlinear function in the nodes inside the reservoir, such as a hyperbolic tangent or a sigmoid. Usually all the nodes have the exact same function. In this study, we introduced a coefficient $\alpha$ in the hyperbolic tangent function. We introduce the heterogeneity in the functions by drawing $\alpha$ from a normal distribution with a mean $\alpha_{\mu}=1$ and various standard deviations $\alpha_{\sigma}$. Here, we fix $\alpha_{\mu}$ and systematically vary the standard deviation of the distribution for $0 \leq \alpha_{\sigma} \leq 5$. We then simulate 100,000 networks and initial conditions to calculate the $H$ and $\lambda$ and study the effect of function heterogeneity on the dynamics of the network. The error bars

are calculated using the standard error $S E=\frac{\sigma}{\sqrt{n}}$, where $\sigma$ is the sample standard deviation and $n$ is the sample size. We find that the function heterogeneity does not have any effect on the dynamics of the LSM. In other words, the LSM dynamics is independent of the heterogeneity in the functions.

\subsubsection{Lyapunov Exponent and Function Heterogeneity}

Here we study the damage spreading $H$ and the Lyapunov exponent $\lambda$ of the LSM as a function of heterogeneity in of the functions in the network. We study $H$ and $\lambda$ for networks with uniform, exponential, and binomial in-degree distributions.

Figure 4.4 shows the damage spreading $H$ as a function of $\alpha_{\sigma}$, the standard deviation of the coefficients $\alpha$ in the network. The network has a Poissonian indegree distribution. For a fixed connectivity $\langle K\rangle, H$ is constant for all values of $\alpha_{\sigma}$. The single step damage spreading is thus independent of the heterogeneity in the function of the system.

Figure 4.5 shows the Lyapunov exponent $\lambda$ of the system for Poisson networks of size $N=100$ for average connectivities $\langle K\rangle=1,2,5$, and 10. Again we see that $\lambda$ is independent of $\alpha_{\sigma}$ for fixed connectivities. We need to remind the reader that LSM with $\langle K\rangle=1$ (blue) are in the ordered regime because $H<1$ (see Figure 4.4), but because we omitted $\lambda=-\infty$ from our calculations, the average 
Lyapunov exponent is $\lambda>0$.

We plot the damage spreading $H$ for LSM of size $N=100$ and connectivity $\langle K\rangle=1,2,5$, and 10 with exponential in-degree distribution in Figure 4.6. Once again, we find that the damage spreading is independent of the function heterogeneity for fixed connectivity $\langle K\rangle$. However, we find that the networks with exponential in-degree have lower values of $H$ than the binomial in-degree for the same values of connectivity $\langle K\rangle$. This result is consistent with the previous conclusions in the last chapter that increased heterogeneity in the in-degree distribution pushes the network dynamics towards the ordered regime.

Finally we plot the Lyapunov exponent $\lambda$ for exponential in-degree distributions in Figure 4.7. $\lambda$ is also independent of the function heterogeneity in exponential networks for fixed connectivity $\langle K\rangle$. The values for $\lambda$ are lower than that of binomial networks with the same $\langle K\rangle$.

To complete this study, we show the $H$ and $\lambda$ for LSM with heterogeneous functions and homogenous in-degrees (uniform in-degree distribution). It is important for us to verify the effects of function heterogeneity in the networks with various in-degree distribution. At least for the extreme values of in-degree heterogeneity, the uniform and the exponential in-degree distributions. We saw that the topological properties of the networks varies dramatically with the in-degree distribution. This difference might cause the collective dynamics of the network to manifest properties in networks with one in-degree distribution that are completely hidden in other in-degree distributions.

Figure 4.8 shows the damage spreading $H$ in the network with uniform in-degree distribution for connectivity $\langle K\rangle=1,2,5,10$. We observe that the $H$ is independent of the function heterogeneity for fixed $\langle K\rangle$ for homogeneous networks as well. The Lyapunov exponent $\lambda$ has the same characteristic that it is independent of the function heterogeneity for fixed $\langle K\rangle$. 
We observe that for binomial networks, homogeneous networks (uniform indegree distribution), and heterogeneous networks (exponential in-degree distribution), damage spreading and the Lyapunov exponent are completely independent of the function heterogeneity $\alpha_{\sigma}$.

Apart from the independence of the $H$ and $\lambda$ of function heterogeneity, there are two observations in these results that are worth addressing. We see that the values for $H$ and $\lambda$ for networks with uniform in-degree distributions, $\langle K\rangle=1$ and 2 collapse onto one single line (Figure 4.8 and Figure 4.9). On the other hand, for exponential networks and the Poisson networks, these values are far part, albeit both in the ordered regime (see Figure 4.4-4.7). In Chapter 3 we saw a similar effect for networks with $\langle K\rangle=1$ and 2 . For both $\langle K\rangle=1$ and 2 , the homogeneous networks showed the exact same value of $H$ and $\lambda$. As we increased the entropy of the in-degree distribution, the values for $H$ and $\lambda$ for these two connectivity level diverged. While the value of both $H$ an $\lambda$ decreased slightly for $\langle K\rangle=2$, the networks with $\langle K\rangle=1$ showed a sharp decrease in the values of the damage spreading $H$ and Lyapunov exponent $\lambda$. This means that the dynamics of the networks with connectivity $\langle K\rangle=1$ is very close to the critical dynamics when the in-degree distribution is uniform. As the in-degree becomes more and more heterogeneous, these networks are pushed into the ordered phase and the values of $H$ and $\lambda$ decrease rapidly. Here we see the same effect for all values of $\alpha_{\sigma}$.

A second key observation is that for all values of $\alpha_{\sigma}$, the values of $H$ and $\lambda$ are lower for exponential networks and binomial networks than that of homogeneous networks with uniform in-degree distribution. The increase in entropy of the indegree distribution pushes $H$ and $\lambda$ for all connectivities toward the ordered regime. This conclusions is also consistent with the results from RBNs (see Chapter 3). 


\subsection{CONCLUSIONS}

In this chapter, we studied the dynamics of RBN and LSM with heterogeneous functions. In the case of $\mathrm{RBN}$, the homogeneous functions reduce the $\mathrm{RBN}$ to a CA with irregular connectivity. The dynamics of such RBN is completely defined by the functions in the system and does not take influence from $p$ and $\langle K\rangle$. As the mixing of different functions in the RBN increases the dynamics of RBN becomes more predictable using $p$ and $\langle K\rangle$. However, we hypothesize that RBN with heterogeneous functions are more suitable for task solving than their homogeneous counterparts in critical regime. This is because the diversity of functions in the heterogeneous RBN help the RBN to decompose features of any input signal in many different ways. This results in better feature extraction ability in heterogeneous RBNs. This hypothesis can be tested in future explorations.

In LSM, we observe that the dynamics is independent of the function heterogeneity for all connectivity levels, and heterogeneity levels in the in-degree distribution. This fact implies that LSM networks show a high resiliency against damage to their functions. We hypothesize that LSM networks may be suitable for information processing in harsh environment in which the node functions might be damaged. Testing this hypothesis is beyond the scope of this thesis and is left for future work. 


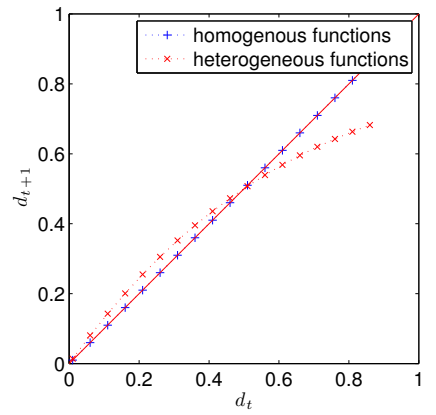

(a) Func 3

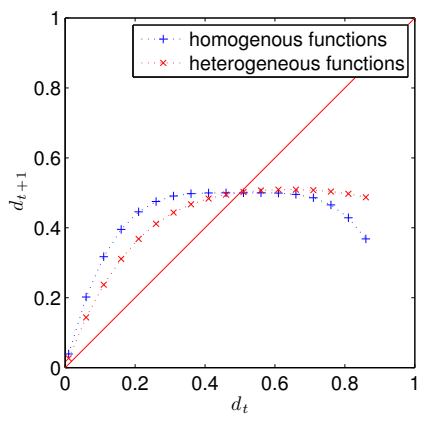

(c) Func 6

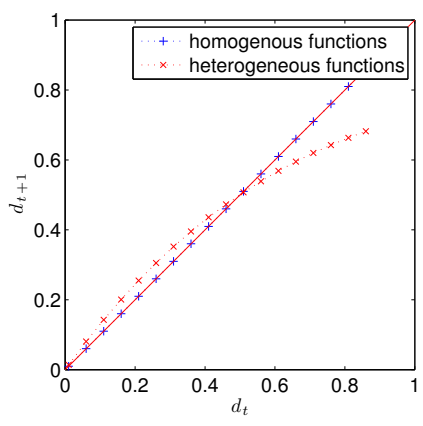

(e) Func 10

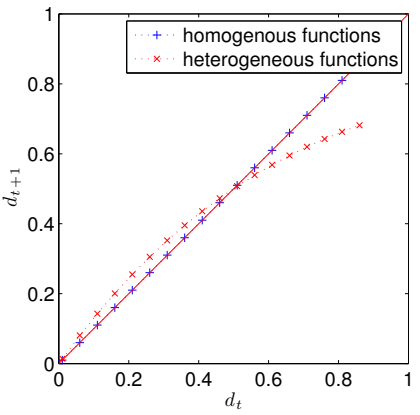

(b) Func 5

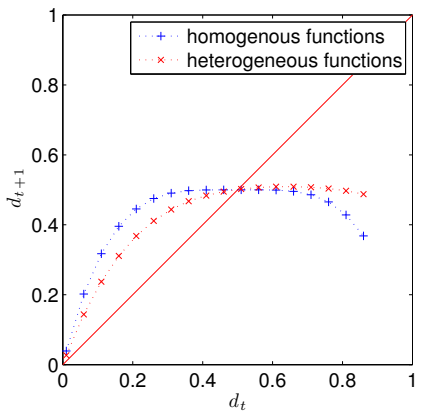

(d) Func 9

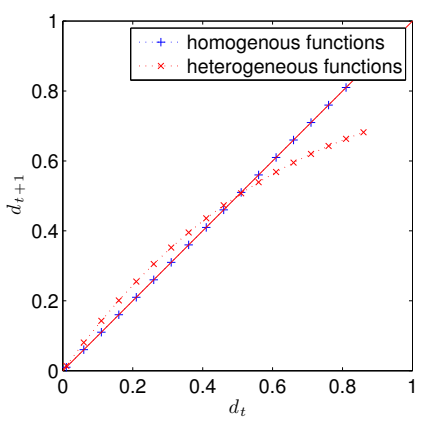

(f) Func 12

Figure 4.3: Derrida curve for RBN networks of uniform in-degree with $K=2$ and bias $p=0.5$ using network functions that are homogeneous, i.e. $\mathcal{H}\left(P_{\mathcal{F}}\right)=0$, and heterogeneous, i.e. $\mathcal{H}\left(P_{\mathcal{F}}\right)=4.96$. 


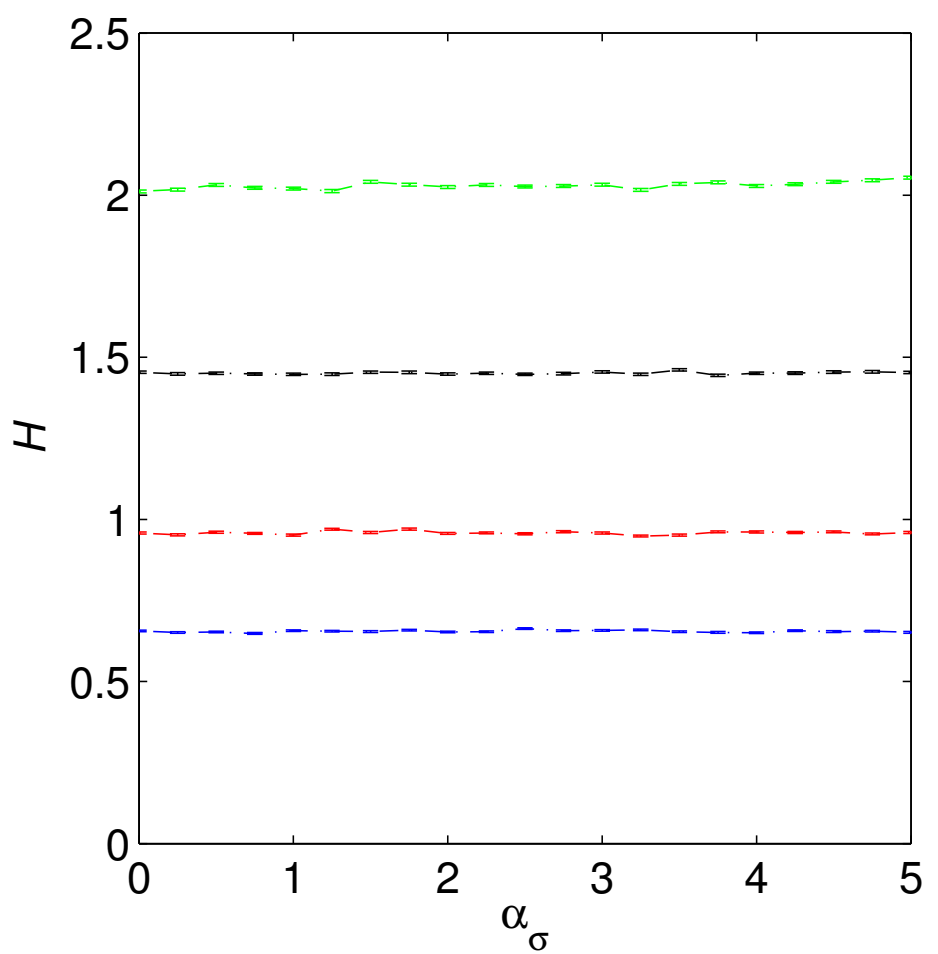

Figure 4.4: Damage spreading $H$ in the LSM with binomial in-degree distribution and heterogenous functions with $\langle K\rangle=1$ (blue), $\langle K\rangle=2$ (red), $\langle K\rangle=5$ (black), $\langle K\rangle=10$ (green). Damage spreading in LSM is independent of function heterogeneity in all connectivity levels. 


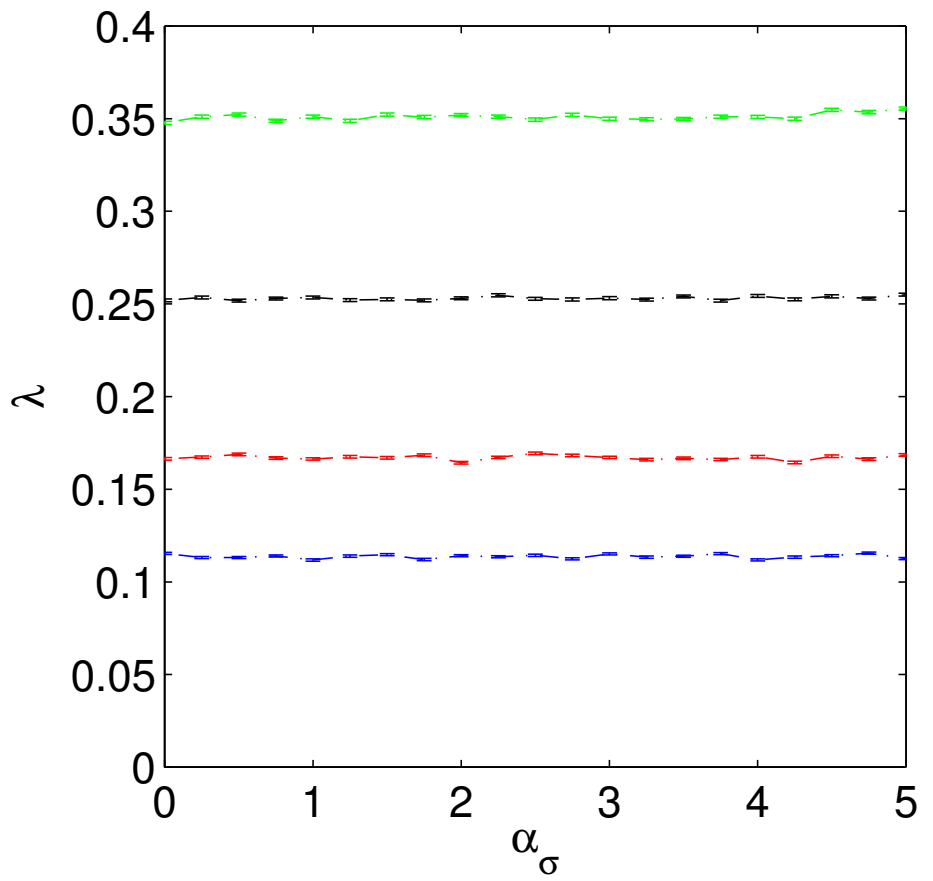

Figure 4.5: Lyapunov exponent $\lambda$ in the LSM with binomial in-degree distribution and heterogenous functions with $\langle K\rangle=1$ (blue), $\langle K\rangle=2$ (red), $\langle K\rangle=5$ (black), $\langle K\rangle=10$ (green). Dynamics of LSM is independent of function heterogeneity in all connectivity levels. 


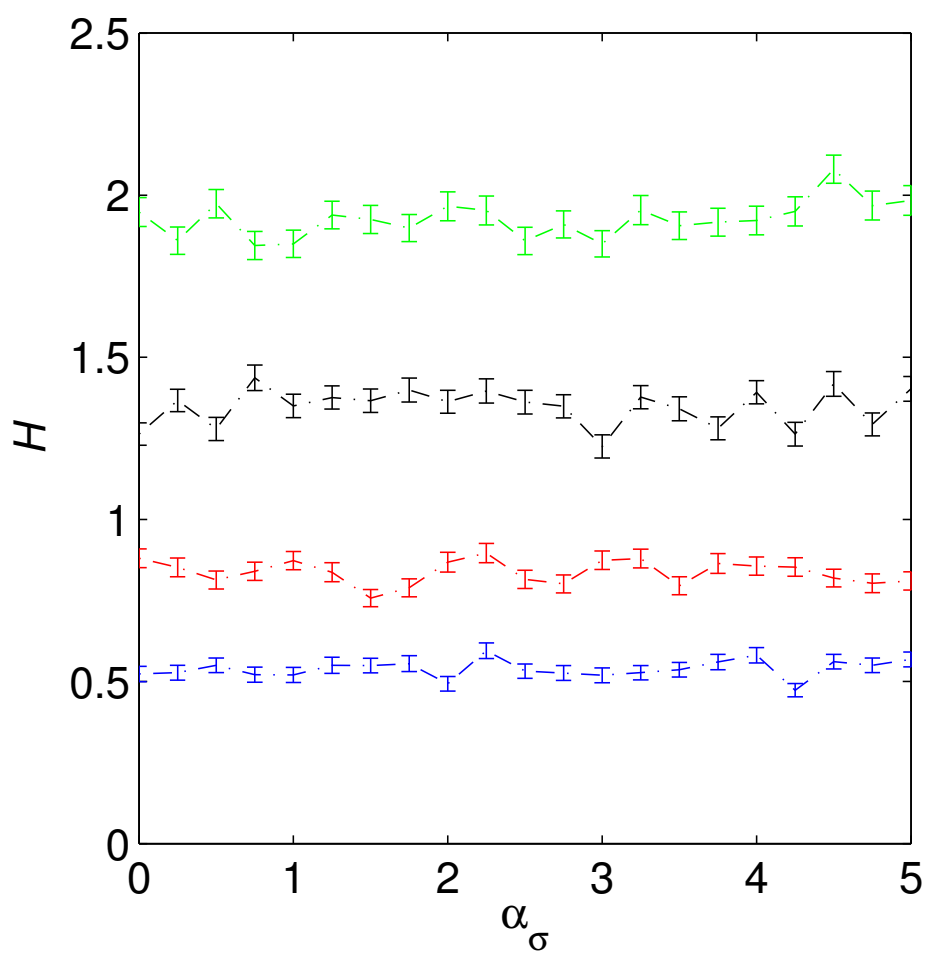

Figure 4.6: Damage spreading $H$ in the LSM with exponential in-degree distribution and heterogenous functions with $\langle K\rangle=1$ (blue), $\langle K\rangle=2$ (red), $\langle K\rangle=5$ (black), $\langle K\rangle=10$ (green). Damage spreading in LSM is independent of function heterogeneity in all connectivity levels. 


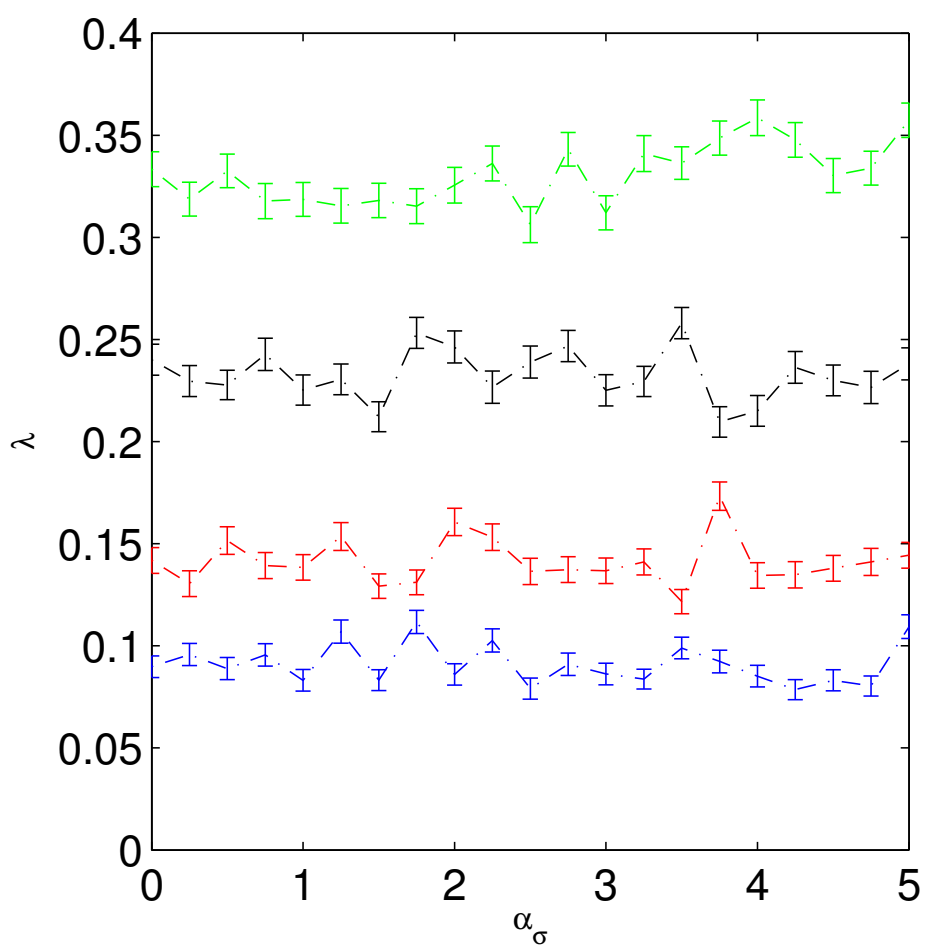

Figure 4.7: Lyapunov exponent $\lambda$ in the LSM with exponential in-degree distribution and heterogenous functions with $\langle K\rangle=1$ (blue), $\langle K\rangle=2$ (red), $\langle K\rangle=5$ (black), $\langle K\rangle=10$ (green). Dynamics of LSM is independent of function heterogeneity in all connectivity levels. 


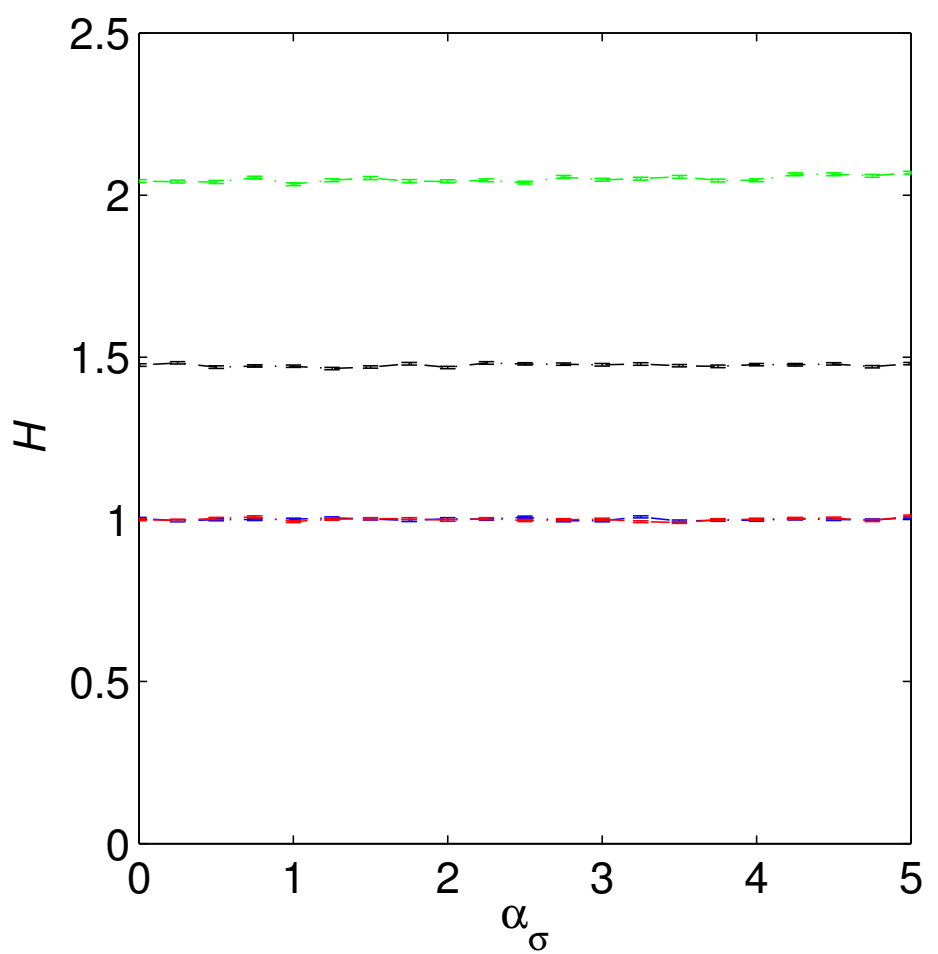

Figure 4.8: Damage spreading $H$ in the LSM with uniform in-degree distribution and heterogenous functions with $\langle K\rangle=1$ (blue), $\langle K\rangle=2$ (red), $\langle K\rangle=5$ (black), $\langle K\rangle=10$ (green). Damage spreading in LSM is independent of function heterogeneity in all connectivity levels. 


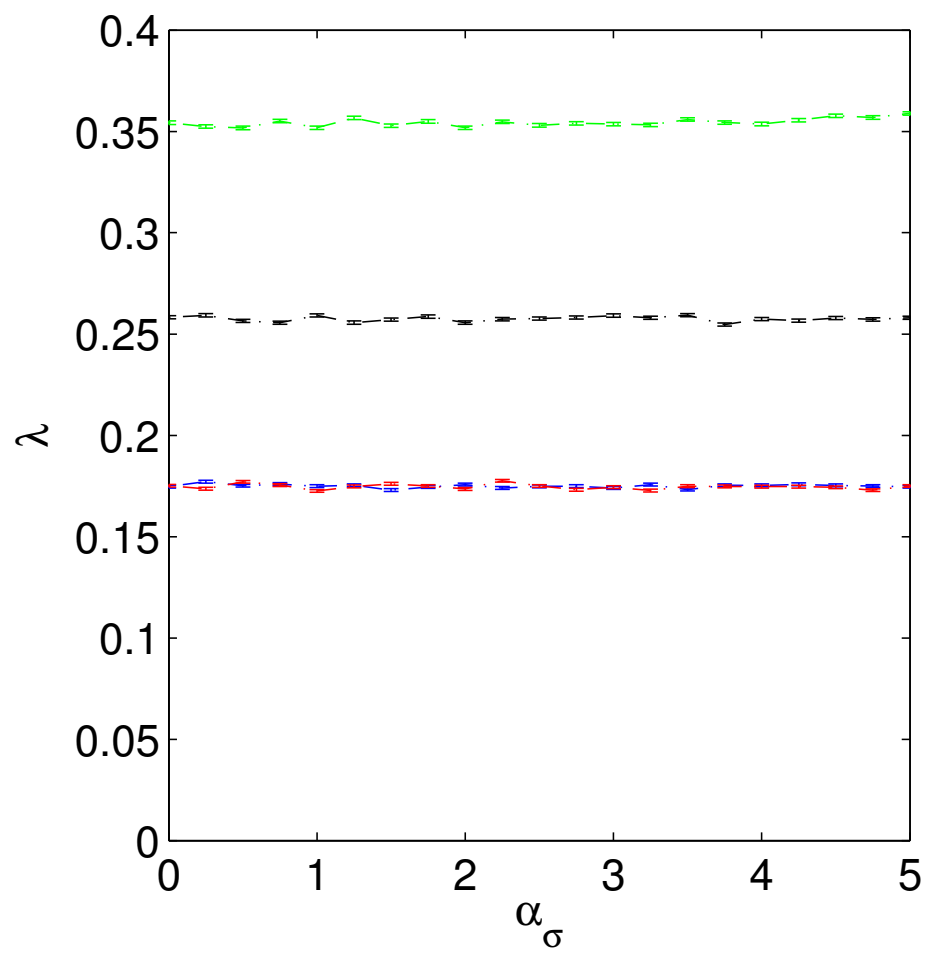

Figure 4.9: Lyapunov exponent $\lambda$ in the LSM with uniform in-degree distribution and heterogenous functions with $\langle K\rangle=1$ (blue), $\langle K\rangle=2$ (red), $\langle K\rangle=5$ (black), $\langle K\rangle=10$ (green). Dynamics of LSM is independent of function heterogeneity in all connectivity levels. 


\section{INTERCONNECT IRREGULARITY}

\subsection{NETWORK LOCALITY}

In conventional LSM and RBN the network structure is a spaceless entity, i.e., in these networks, there is no distance between the nodes and the connections have no length. However from an engineering perspective, we are interested in the physical realizability of RBN and LSM networks. In this chapter, we introduce spatial LSMs and RBNs by embedding the underlying network of in a 2-dimensional lattice. A similar approach has been used in $[23,73,87]$ to study the critical behavior of smallworld and spatial dynamical networks. As described in Chapter 2, we embed the network nodes on a 2 -dimensional lattice with the typical length $l=1$. We then wire the nodes together according to a pre-defined in-degree sequence $\psi$ so that the probability of a link between nodes $i$ and $j$ is a function $F$ of the distance matrix $D_{M}=\left\{r_{i j}\right\}[23]$. This will give rise to a sequence of wire lengths $\mathcal{L}_{a}$ for every value of $a$. Consistent with the heterogeneity in the functions and the in-degree distribution, we choose to measure the interconnect irregularity using the entropy of the distribution of the wire lengths $\mathcal{H}\left(P_{\mathcal{L}_{a}}\right)$.

The form of $F$ depends on a particular application. We are interested in the physical realizability of self-organized networks. It has been shown that in selforganized networks the length distribution between nodes follows a power-law [87]. Therefore, we follow the same model and use a power-law, i.e., $F\left(l_{i j}\right)=r_{i j}^{-a}$ to define our wire length distribution:

$$
P_{l_{i j}}=r_{i j}^{-a}
$$


Figure 5.1 shows the wire length probability distribution $P_{\mathcal{L}_{a}}$ for different values of $a$ and $K=1,2,3$, and 4 . For $a=0.0$, we have $P_{l_{i j}}=r_{i j}^{0.0}=1$ and therefore the connection between any two pairs of nodes is independent of their distance. Thus the distribution $P_{\mathcal{L}_{a}}$ takes the form of a Poissonian distribution. Entropy of this distribution is $\mathcal{H}\left(P_{\mathcal{L}_{a}}\right) \approx 3.3$. As we increase $a$, the probability distribution becomes skewed to the left and for $a \geq 1$ takes the form of a power-law. The entropy of the distribution decreases as power-law distribution becomes sharper. For $a=4$, the entropy of the wire length distribution $\mathcal{H}\left(P_{\mathcal{L}_{a}}\right) \approx 1.25$.

Figure 5.2 shows entropy of the wire length distribution $\mathcal{H}\left(P_{\mathcal{L}_{a}}\right)$ as a function of $a$ for network size $N=100$ and connectivity $K=1,2,3$, and , 4 for networks with uniform in-degree distribution. The entropy values are averaged over 1000 networks. We observe that for all the $K$ values the entropy curves are the same qualitatively and show the same behavior. For $K=1$, the entropy values are smaller than entropies for $K>1$, however, the difference is smaller than 0.1 and does not have any significant effect in our study. Moreover, $\mathcal{H}\left(P_{\mathcal{L}_{a}}\right)$ is just a function of the probability distribution of the wire lengths. The change in $K$ only changes the number of available wires $N \times K$ on the network and not their distribution. As $N$ and $K$ increase, $N \times K$ approaches the thermodynamic limit and thus wire length distribution becomes ideal. The difference between this wire length distribution for lower values of $N \times K$ therefore is not fundamental. Thus for our purpose, $\mathcal{H}\left(P_{\mathcal{L}_{a}}\right)$ is independent of $K$.

From the perspective of heterogeneity, we see that $\mathcal{H}\left(P_{\mathcal{L}_{a}}\right)$ and $a$ have an inverse relation, i.e., the maximum and the minimum of $\mathcal{H}\left(P_{\mathcal{L}_{a}}\right)$ coincide, with the lowest and highest value of $a$ respectively. This means that for $a$ we have the most heterogeneous system and for $a=5$ we have the most homogeneous system in terms of wire lengths.

From the locality point of view, for $a=0$, we have all the different connection lengths realized independently and therefore the network looses the spatial 
relationship. Such a network is equivalent to the Erdös-Rényi random graph. For $a \rightarrow \infty$, the connections are all local and therefore we have a completely ordered lattice of connections. For finite positive values of $a$, the connectivity is smallworld, in which there are combination of long-range and short-range connections.

Our view of heterogeneity here is not shared by all authors [106]. If one is interested in simply the cardinality of different wire lengths, the power-law distribution would provide the most heterogeneous distribution. However as we described in Chapter 2, having numerous wire lengths does not mean that in any practical application we observe the diversity of different lengths. Therefore, in our study, the power-law distribution signifies a homogeneous system and, Poissonian wire length distribution is the heterogeneous system.

\subsection{NETWORK TOPOLOGY}

A dominant factor in network dynamics is the structure or the topological properties of the network. We previously saw that many of the dynamical properties of the network can be explained using the effects of heterogeneity in the topological properties of the network. It is through this topological effects that the heterogeneity influences the dynamics of the network. In this section, we first study the effects of the wire length heterogeneity in the relevant topological measures of the network. We then derive some expectations about how these measures should affect the dynamics of the network as a function of the entropy of the wire length $\mathcal{H}\left(P_{\mathcal{L}_{a}}\right)$. In the next section we show the result of the dynamics and draw a connection between the results and network measures.

One of the most important and the most studied network measures is the local clustering coefficient in the network [94]. This measure is calculated for each node on the network. This measure indicates how closely adjacent nodes in a network are connected together (see Chapter 2). This measure is particularly important for dynamical processes on the networks. In a neighborhood of a network 
where the nodes have high clustering coefficient, the information percolates from many different paths and might interfere with itself. Figure 5.3 shows the average local clustering coefficient for networks of size $N=100$ and connectivity $K=$ $1,2,3$, and , 4 as a function of $a$ and $\mathcal{H}\left(P_{\mathcal{L}_{a}}\right)$. For random networks, i.e. $a=0$ and $\mathcal{H}\left(P_{\mathcal{L}_{a}}\right)=3.25$, the mean clustering coefficient is minimal. This is a well-known effect and not surprising since the probability of nodes being connected together is independent of nodes' distance. For local networks on the other hand, i.e. $a=5$ and $\mathcal{H}\left(P_{\mathcal{L}_{a}}\right)=1$, the clustering coefficient is at it is maximum 0.2 for $K=1$ networks and near maximum 0.6 for $K>1$. This means the ability of information transfer in clusters of nodes decreases linearly as the heterogeneity of wire lengths increases. This may imply that wire length heterogeneity imposes order on network dynamics.

Another well-studied measure is the average shortest path [94]. This measure indicates on average how easily pairs of nodes can be reached from each other by traveling through the network. The shorter this distance is, the faster a signal can spread in the network and therefore information propagation is more efficient. Figure 5.4 shows the average shortest path for networks of size $N=100$ and connectivity $K=1,2,3$, and 4 as a function of $a$ and $\mathcal{H}\left(P_{\mathcal{L}_{a}}\right)$. This behavior of the average shortest path is more complex than the clustering coefficient. For $K=1$ (blue), we observe that for random networks, i.e., $a=0$ and $\mathcal{H}\left(P_{\mathcal{L}_{a}}\right)=3.25$, the average shortest path length is at its maximum $(\approx 7)$ and decreases quadratically. As the wire lengths become more homogeneous and the connections become more local, the average shortest path decreases and reaches the minimum value, i.e., 2 for completely homogeneous system $\mathcal{H}\left(P_{\mathcal{L}_{a}}\right)=1$. This is because for $\langle K\rangle=1$, the network is very sparse and the formation of strongly connected components only occurs in highly local networks. On the other hand, for $\langle K\rangle>1$, the average shortest path decreases as the wire length becomes more and more heterogeneous and reaches its minimum for $\mathcal{H}\left(P_{\mathcal{L}_{a}}\right)=3.25$. Note that $1 \leq \mathcal{H}\left(P_{\mathcal{L}_{a}}\right) \leq 3.25$ is 
the absolute range for wire length heterogeneity in this study and therefore the maximum and minimums of the average shortest path are the global maxima and minima. A naive interpretation is that for $\langle K\rangle=1$, increased heterogeneity in the wire length should make this system more chaotic, whereas for $K>2$, increased heterogeneity should impose order. In the next sections, we will see how the average shortest path actually influences the dynamics.

A closely related measure to the clustering is the $Q$ value introduced by Newman [83]. This value is a measure of cohesiveness of modules in the network. For random networks with no modules this value should be very low or zero and for highly modular networks $Q$ should be large and close to 1 . Figure 5.5 shows the Q value for networks of size $N=100$ and connectivity $K=1,2,3$, and 4 as a function of $a$ and $\mathcal{H}\left(P_{\mathcal{L}_{a}}\right)$. As expected for local networks of all connectivities, i.e., $\mathcal{H}\left(P_{\mathcal{L}_{a}}\right)=1$, the $Q$ value is at its maximum and drops as the heterogeneity increases. For random networks, i.e., $\mathcal{H}\left(P_{\mathcal{L}_{a}}\right)=3.25, Q$ is at its minimum, implying that the observed modules in the network are not very cohesive. This usually means that the nodes in the modules, have a lot of links to other nodes in other modules and therefore the modularity is weak. In principle the behavior of the $Q$ value should track the behavior of the local clustering coefficient. In fact, we see that the qualitative behavior of $Q$ as a function of $\mathcal{H}\left(P_{\mathcal{L}_{a}}\right)$ (Figure 5.5(b)) is very similar to how the clustering coefficient changes as a function of the entropy (Figure 5.3(b)). For $K=1$ we see a completely linear decrease of $Q$ and the clustering coefficient as heterogeneity increases. For $K>1$, both $Q$ and the clustering coefficient show a sharper and non-linear decrease with increasing heterogeneity. Therefore, with higher connectivity the networks lose their module features faster as the system become heterogeneous in wire lengths. 


\subsection{DYNAMICS OF RANDOM BOOLEAN NETWORKS}

We have seen in Chapter 4 and Chapter 5 that the RBN dynamics are sensitive to both heterogeneity in the in-degree distribution and the function heterogeneity. Here we study the dynamics of RBN for conventional RBN networks with homogeneous in-degree distributions as a function of the entropy of the wire length distribution $\mathcal{H}\left(P_{\mathcal{L}_{a}}\right)$. Damage spreading in RBN has been studied and it has been known that for local networks the dynamics are chaotic [73]. As the network becomes more small-world, the dynamics becomes critical. In addition, small-world networks in both $\mathrm{RBN}$ and $\mathrm{CA}$ improve the computational performance of the network in solving the density task $[76,110]$. We investigate this phenomena by studying the dynamics of the RBN as a function of $\mathcal{H}\left(P_{\mathcal{L}_{a}}\right)$ and we will show how the small-world effect regulates the dynamics in the network.

To study the dynamics we use the Derrida measure to calculate how fast the damage spreads in the RBN. We experiment with networks of average connectivity $\langle K\rangle=1.0,1.5,2.5$, and 3.0. Initially all the network samples have random connectivity with $a=0$ and are therefore equivalent to random networks. We vary $a$ and calculate the entropy of the distribution of the wire length. We average the results over 1,000 runs and express the result in terms of the entropy values. We express the result as how the logarithm of $\frac{d_{t+1}}{d_{t}}$ changes with $\mathcal{H}\left(P_{\mathcal{L}_{a}}\right)$. For chaotic networks, $\log \left(\frac{d_{t+1}}{d_{t}}\right)>0$, for ordered networks, $\log \left(\frac{d_{t+1}}{d_{t}}\right)<0$, and for critical networks, $\log \left(\frac{d_{t+1}}{d_{t}}\right)=0$.

The plots in Figure 5.6 show the summary of our findings for RBN with different average connectivities. We see that for $\langle K\rangle>2$, the networks are chaotic as expected and for $\langle K\rangle<2$ the networks are in the ordered regime. Moreover for ordered networks the dynamics become more ordered as the heterogeneity increases. On the other hand, for chaotic networks, as the heterogeneity increases, the network becomes more chaotic. In both chaotic and ordered networks the 
heterogeneity in the wire lengths pushes the networks even further away from the desirable critical regime.

\subsection{DYNAMICS OF LIQUID STATE MACHINES}

In this section we study how the heterogeneity in the wire length affect the one time step divergence $H$ and the Lyapunov exponent $\lambda$ in liquid state machines. So far we have seen that the in-degree heterogeneity makes the LSM dynamics more ordered whereas the function heterogeneity does not have any effect on the dynamics. Here we use LSM networks of size $N=100$ and connectivity $K=1,2,3$, and 4 with homogeneous in-degrees and functions. We study the dynamics by calculating $H$ and $\lambda$ for LSM with $0 \leq a \leq 5$. We average the results over 1,000 samples.

Figure 5.7(a) shows the average $H$ as a function of $a(\mathrm{a})$ and $\mathcal{H}\left(P_{\mathcal{L}_{a}}\right)$ (b). Data for networks of $K=1,2,3$, and 4 is plotted in blue, black, magenta, and red respectively. The error bars represent the standard error. We observe that aside from small fluctuations, the value of $H$ is constant and independent of $a$ and $\mathcal{H}\left(P_{\mathcal{L}_{a}}\right)$. Both $K=1$ and $K=2$ networks have identical values very close to $H=1$, which means according to $H$, these systems are in the critical phase. For $K=3$ and $4, H>1$, and therefore these systems are chaotic. We see that the connectivity $K$ does play a significant role in the chaotisity of the network measured by $H$, but not by the heterogeneity of the wire lengths.

Figure 5.8(a) shows the Lyapunov exponent $\lambda$ for the same samples of LSM networks described above. We see that $\lambda$ can differentiate between the network dynamics of networks with different wire length heterogeneity (Figure 5.8(b)). For all $K$, a higher heterogeneity imposes order on the network dynamics. We see that $\lambda$ decreases linearly with $\mathcal{H}\left(P_{\mathcal{L}_{a}}\right)$.

It is unintuitive that more heterogeneous wire lengths cause more ordered dynamics. Petermann et al. [87] studied the relationship between $a$ and the node connectivity in lattice and small-world networks. They argued that for positive 
values of $a$ the network becomes more small-world. This small-world regime is characterized by a high clustering coefficient (feature of local network) and a low average path length (feature of random networks) [113]. The improved navigability in small-world networks is therefore attributed to the existence of different connection length scales rather than just a small average path length. Lu and Teuscher [73] investigated the relation between the small-worldness and the damage spreading and showed that the damage spreads faster in local networks. This is due to high clustering between nodes in networks with local connections that percolate damage in local neighborhoods quickly. These findings agree with our results.

For $a=0$, the network is completely random and has the maximum heterogeneity in the wire lengths. Networks of this type have a very short average path length and a very low clustering. The perturbation in one area might quickly spread in the entire network, since the percolation spreads in all different routes, they might cancel each other's effect. This results in lower damage spreading values for fixed $K$. For positive values of $a$, both clustering increases and $Q$ decreases which implies highly connected modules in the network forms. But these modules are also connected to other modules across the networks meaning that any perturbation both spreads in the modules and between modules. Homogeneity in wire length, therefore, result in richer and perhaps chaotic dynamics.

It is interesting that both for in-degrees and wire length, more heterogeneity in the network imposes order in the dynamics. Can we find out what the optimal connectivity $K_{c}$ is for minimum and maximum heterogeneity in the wire lengths? That is, at what $\langle K\rangle=K_{c}$ the system dynamics becomes critical, i.e., $\lambda=0$. Figure 5.9 shows the Lyapunov exponent as a function of $\langle K\rangle$ for networks of size $N=100$ with homogeneous in-degree distributions. The red curve shows for local networks with homogeneous wire length distributions and the blue curve random networks with a maximal heterogeneous wire length. The postulation that 
heterogeneity imposes order on the network becomes clear in this plot. We can calculate the exact critical connectivity $\langle K\rangle=K_{c}$ for which $\lambda=0$. For the interval $2 \leq\langle K\rangle \leq 3$, the change in $\lambda$ is linear. Using linear regression, we can fit the linear model:

$$
\lambda(\langle K\rangle)=a\langle K\rangle+b
$$

For random networks, i.e., $\mathcal{H}\left(P_{\mathcal{L}_{a}}\right)=3.25$ (blue), $a=0.12$ and $b=-0.35$. For local networks, i.e., $\mathcal{H}\left(P_{\mathcal{L}_{a}}\right)=1$ (red), $a=0.13$ and $b=-0.34$. We therefore calculate the critical connectivity for random networks with homogeneous in-degree and heterogeneous wire lengths as:

$$
K_{c}^{\text {heterogeneous }}=2.91 \text {. }
$$

The critical connectivity for local networks with homogeneous in-degree and homogeneous wire lengths is:

$$
K_{c}^{\text {homogeneous }}=2.61
$$

Since the heterogeneous wire length networks are more ordered, they need a higher connectivity to achieve critical dynamics in the system. If one wants to build such a network, one would need more connections per node on average, which increases the direct relation between connectivity per node and cost.

\subsection{CONCLUSIONS}

We investigated the dynamics of RBN and LSM as a function of heterogeneity in the wire lengths. For RBNs, the higher wire length heterogeneity results in richer dynamics, where RBN is pushed towards the critical regime. For LSM, the chaotic systems are pushed towards the critical region as the wire length heterogeneity increases. Critical systems, however, become more ordered and therefore not suitable for computation. 


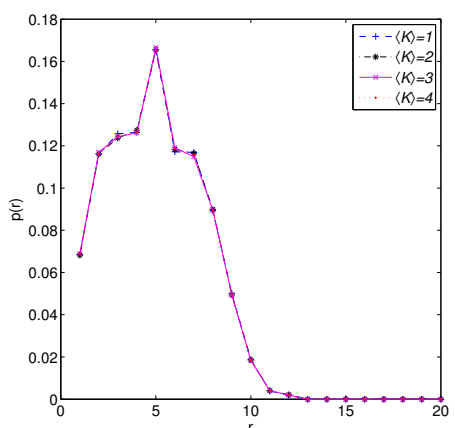

(a)

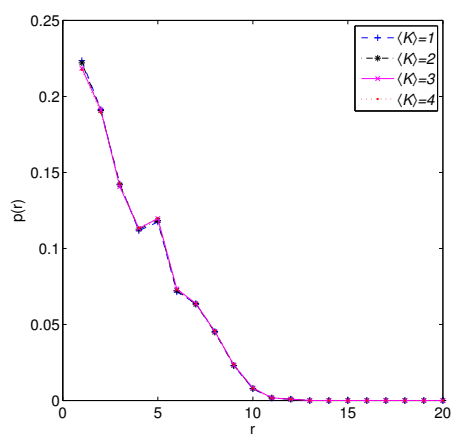

(c)

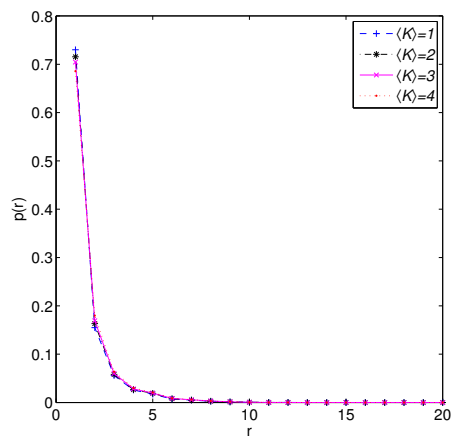

(e)

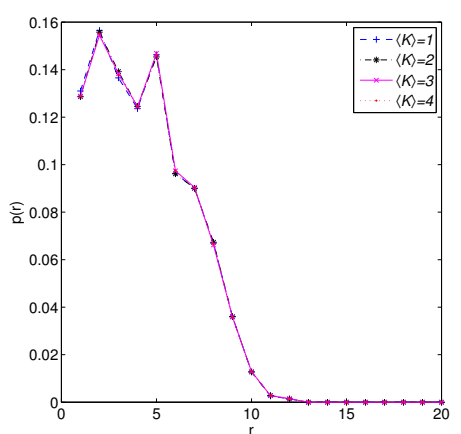

(b)

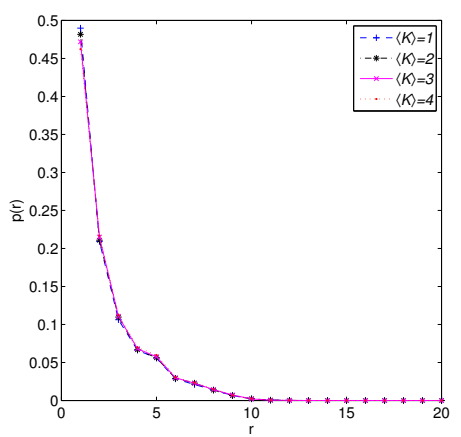

(d)

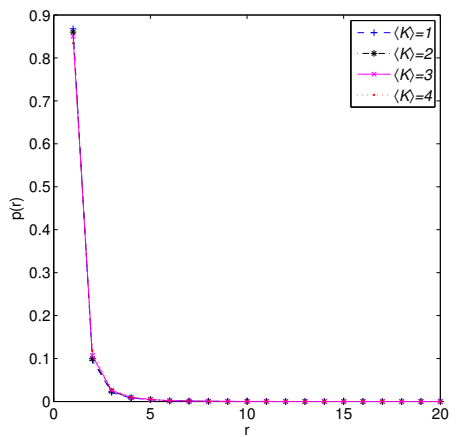

(f)

Figure 5.1: Wire length probability distribution $P_{\mathcal{L}_{a}}$ for $a \in$ 0.0, 0.5, 1.0, 2.0, 3.0, 4.0. 


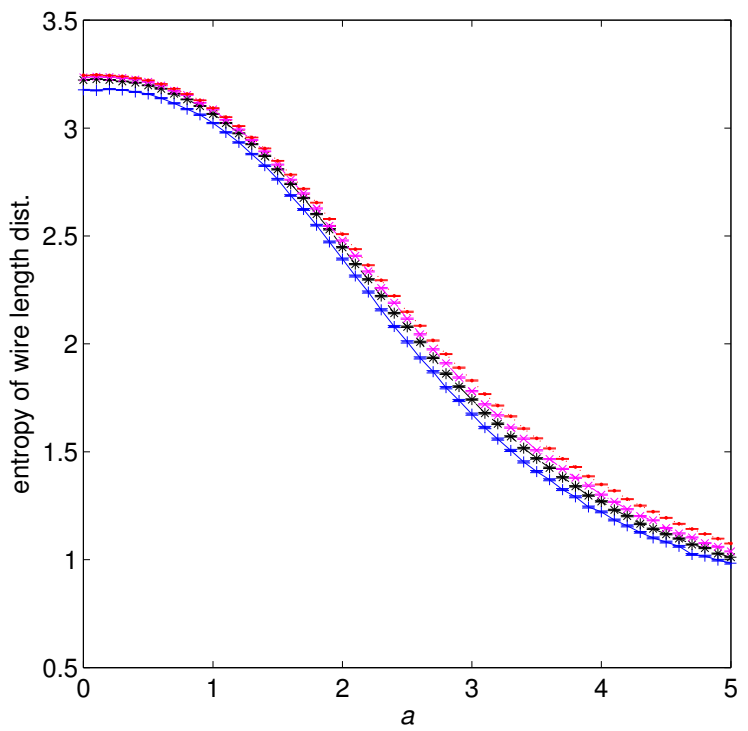

Figure 5.2: Entropy of the wire length distribution $\mathcal{H}\left(P_{\mathcal{L}_{a}}\right)$. The entropy of the wire lengths shows a second order phase transition behavior, in which for global networks the entropy is maximal while it shows a sudden decrease for more locally connected networks. Global networks are more heterogeneous and local networks are more homogeneous with respect to the wire lenghts. 


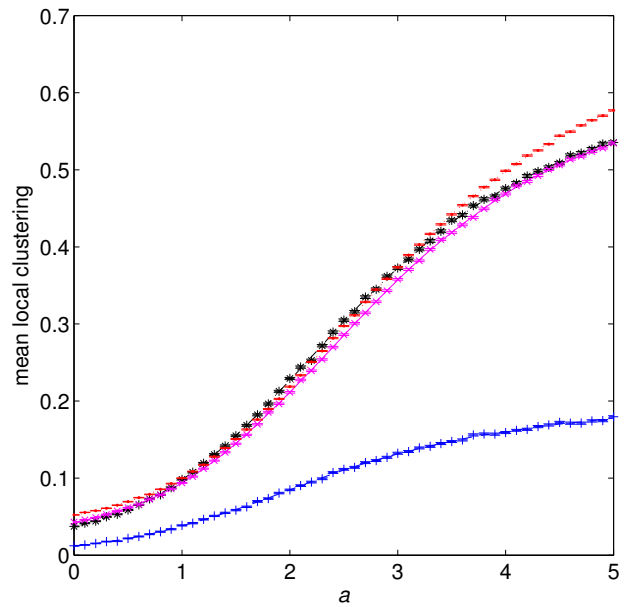

(a)

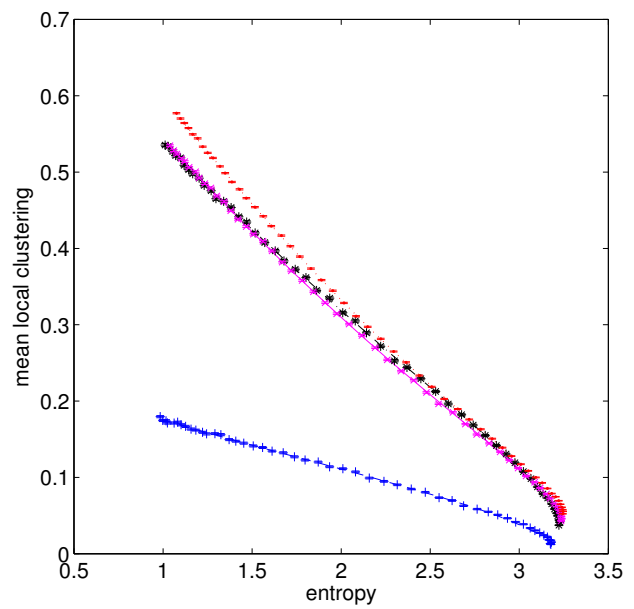

(b)

Figure 5.3: Average local clustering in the networks as a function of $a(\mathrm{a})$ and $\mathcal{H}\left(P_{\mathcal{L}_{a}}\right)(\mathrm{b})$. Blue curve is $K=1$, black curve is $K=2$, magenta curve is $K=3$, and red curve is $K=4$.

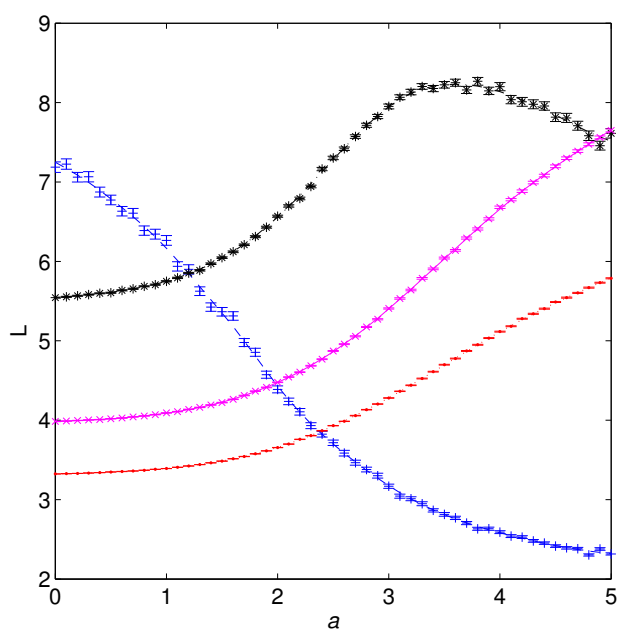

(a)

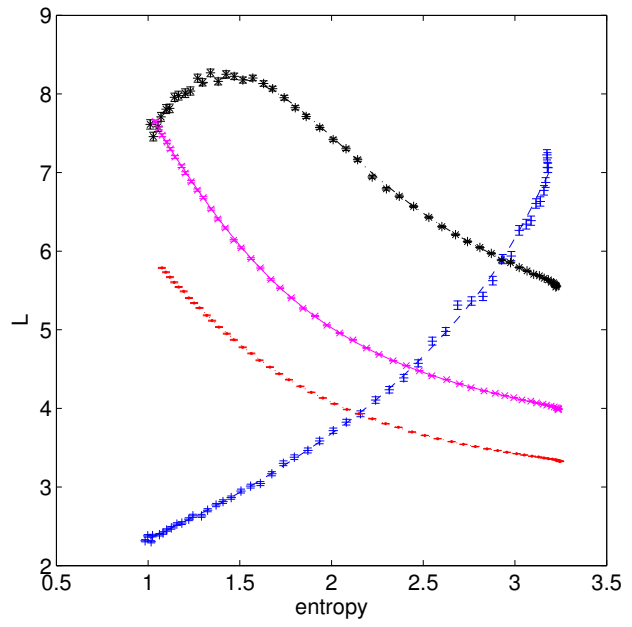

(b)

Figure 5.4: Average shortest path length $L$ in the networks as a function of $a(\mathrm{a})$ and $\mathcal{H}\left(P_{\mathcal{L}_{a}}\right)(\mathrm{b})$. Blue curve is $K=1$, black curve is $K=2$, magenta curve is $K=3$, and red curve is $K=4$. 


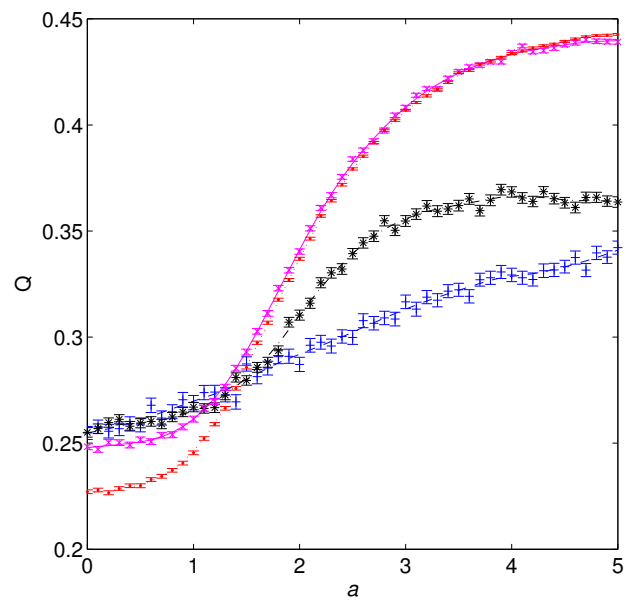

(a)

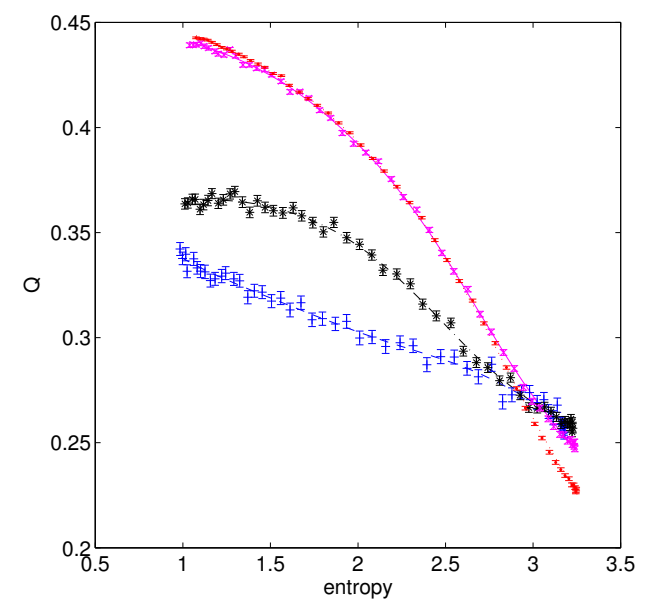

(b)

Figure 5.5: Average modularity $Q$ value in the networks as a function of $a($ a) and $\mathcal{H}\left(P_{\mathcal{L}_{a}}\right)(\mathrm{b})$. Blue curve is $K=1$, black curve is $K=2$, magenta curve is $K=3$, and red curve is $K=4$. 


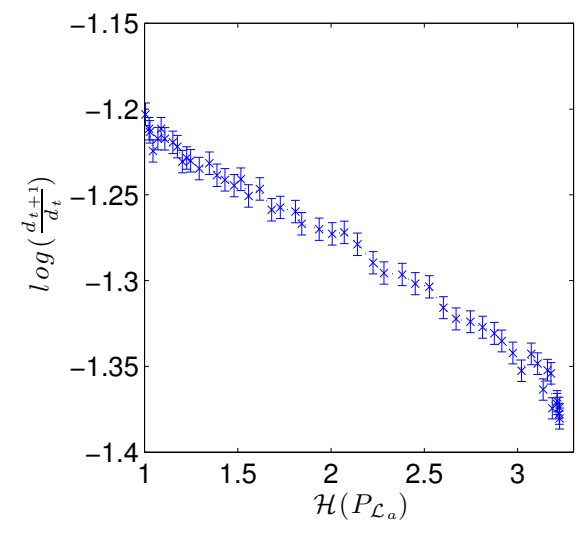

(a) $\langle K\rangle=1.0$

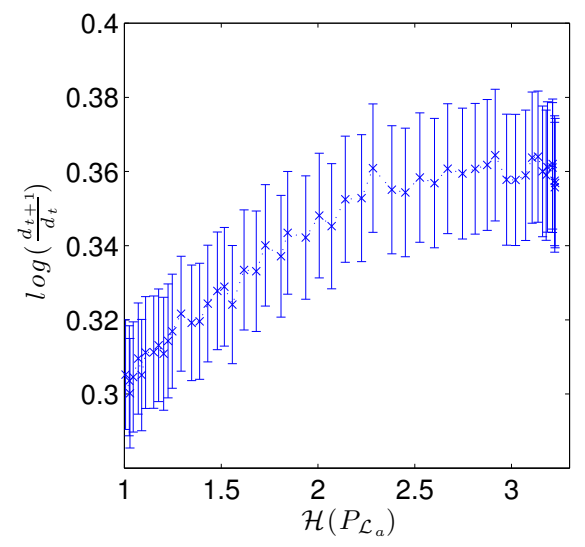

(c) $\langle K\rangle=2.5$

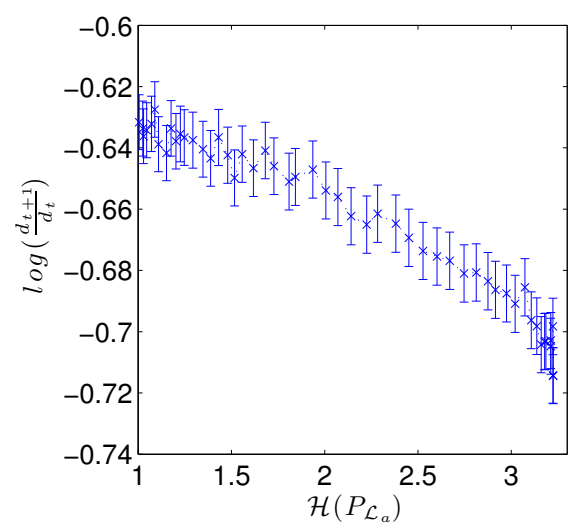

(b) $\langle K\rangle=1.5$

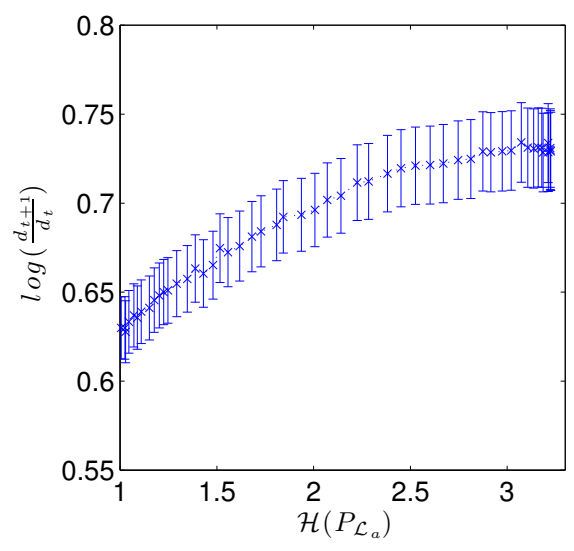

(d) $\langle K\rangle=3.0$

Figure 5.6: Dynamics of RBN with different connectivity as a function of entropy of wire length distribution. Increasing heterogeneity in the networks pushes the RBN dynamics towards more critical region causing richer dynamics suitable for information processing. 


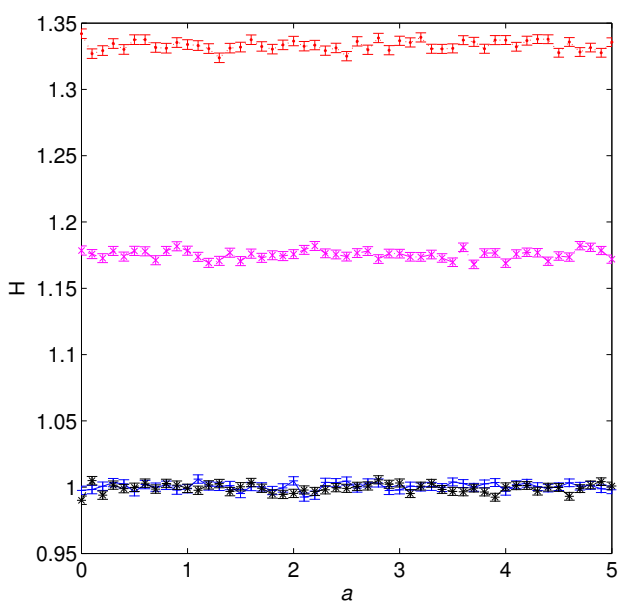

(a)

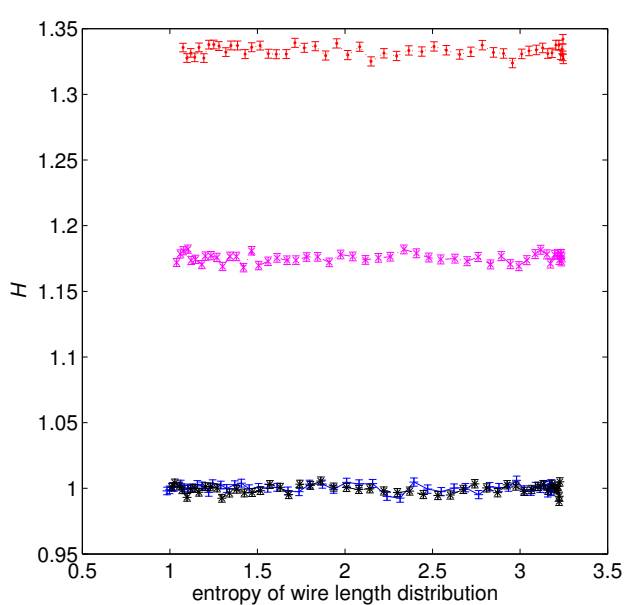

(b)

Figure 5.7: Damage spreading $H$ as a function of $a(\mathrm{a})$ and $\mathcal{H}\left(P_{\mathcal{L}_{a}}\right)$ (b). Damage spreading does not show any effect due to heterogeneity of the wire lenghts.

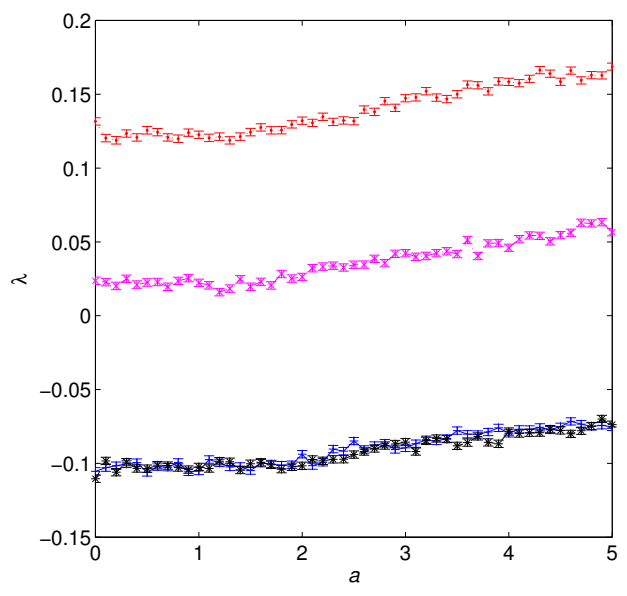

(a)

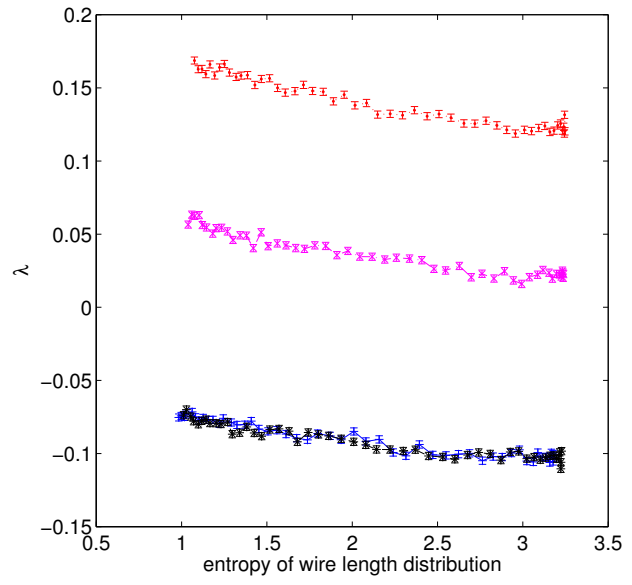

(b)

Figure 5.8: Lyapunov exponent $\lambda$ as a function of $a(\mathrm{a})$ and $\mathcal{H}\left(P_{\mathcal{L}_{a}}\right)$ (b). Dynamics of LSM become poorer as the heterogeneity of the wire lengths increase. Chaotic systems are pushed towards critical regime while the critical systems are pushed towards ordered regime. 


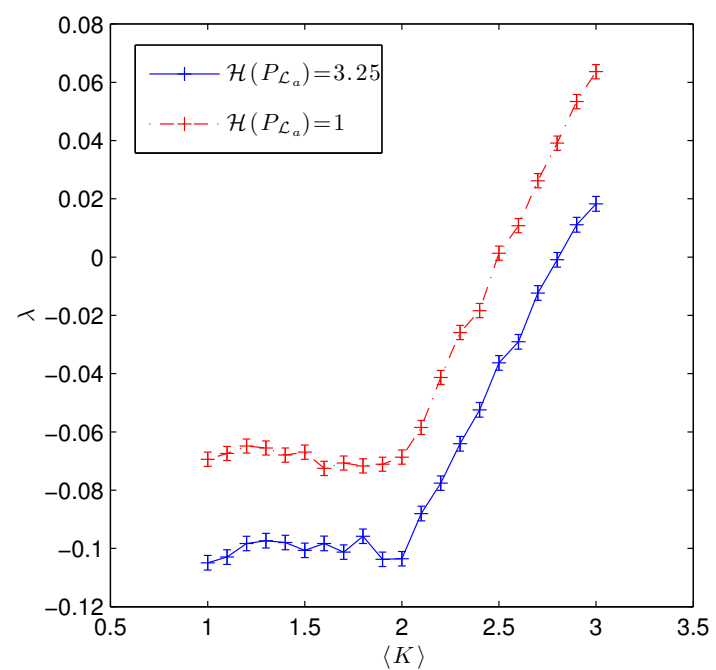

Figure 5.9: $\lambda$ as a function of $\langle K\rangle$ for $\mathcal{H}\left(P_{\mathcal{L}_{a}}\right)=3.25$ (blue) and $\mathcal{H}\left(P_{\mathcal{L}_{a}}\right)=1$ (red) networks. 


\section{CONCLUSIONS}

In this thesis, we conducted a systems study of the effects of heterogeneity on the dynamics of random dynamical networks. We carried this study within the framework of systems approach to identify the context and the problem domain, and to analyze the problem statement. Using our insight, we then chose two models to continue our study. Although the models in this study are much simplified, they carry the essential systems behavior pertinent to our study. Moreover, both models consist of relevant components that we investigated to achieve the goal of this study, that is to understand the relationship between the changes in the systems behavior with respect to the changes in the properties of the components. A summary of this study, our methods, and the results is presented here.

This study was motivated by the need for alternative computing paradigms. These novel computing paradigms must be both cheap to make and programmable. One approach that has recently gained much attention is called reservoir computing. In reservoir computing, one would stimulate an excitable physical system and then interpret the intrinsic dynamics of the system to produce the desired output for the computation. Since this approach relies only on the dynamical behavior of physical systems, in principle, any system could be used to implement this paradigm. Specifically, reservoir computing is suitable to compute with self-assembled molecular and nanoscale devices.

The focus of research in reservoir computing is finding systems with suitable dynamic for computation. The dynamics of any physical system can be attributed

to its interacting parts. Therefore, a scientific study of reservoir computing in 
physical systems using a systems perspective lend itself to employing dynamical network models. Network models make it possible to study the global systems behavior with respect to the interacting components of the system. The property of interest in our study was the heterogeneity in the components of the system and their interactions. The global dynamics of the system in dynamical networks depend on change in the node state from each point in time to the next. For each node, the change in the state is governed by the node's transfer function. The output of the node function in turn depends on the interaction between the state of other nodes in the previous time step. Our aim was to study how the heterogeneity of the node functions, the in-degree of the nodes, and the wire lengths of the connections between the nodes affect the systems dynamics.

We chose two classical dynamical network models called random Boolean network $(\mathrm{RBN})$, which is a model of gene regulatory networks, and liquid state machine (LSM), which is a model of cortical microcircuits. Both models have been used to implement reservoir computing. Most of the work in reservoir computing use the homogeneous networks. However, homogeneity in real physical systems is rare to find. We bridge the gap in this research by systematically introducing heterogeneity in the networks and study how this heterogeneity affects the systems' dynamics.

In this thesis we aimed at understanding the effects of heterogeneity on the dynamics of two network models, LSM and RBN, which represent a class of physical systems. We introduced entropy as a generic measure of heterogeneity for complex networks, which can measure heterogeneity in any aspect of a network describable using a nominal variable. For our purpose, we investigated heterogeneity in the node functions, the node in-degrees, and the wire lengths. We showed that entropy is predictive of the effects of heterogeneity on the dynamics of the network. Our findings in the course of this thesis is summarized in Table 6.1. For comparison 
we have also included existing results in the literature on the effects of the indegree heterogeneity on the dynamics of coupled oscillators (compared to LSM) and scale-free RBNs (compared to our RBN).

In LSM, heterogeneity in node in-degree and wire lengths result in poor system dynamics. This implies that for LSMs systems with high a level of heterogeneity in the in-degrees and the wire lengths, the critical connectivity to reach the critical dynamical regime increases. This increased connectivity will compensate for the imposed orderly behavior induced by the heterogeneity on the network. The node function heterogeneity has no effect on the dynamics of the LSM. This might be due to the weighted nature of the connections and the insensitivity of the collective dynamics to the exact value of the derivative of the node functions in LSMs. This will require more in-depth study of these networks.

For RBN, we observe that the in-degree heterogeneity and the wire length heterogeneity lead to richer dynamics. We observed that function heterogeneity in RBNs result in predictably richer dynamics. If one can choose the functions of all the nodes accurately, it is possible to construct RBNs with homogeneous functions with rich dynamics as well. However, we hypothesize that since for online computation using RBN each node may act as a filter to decompose external inputs, RBNs with heterogeneous functions may outperform homogenous RBNs.

Our result in in-degree heterogeneity in both RBN and LSM agrees with existing result on the dynamics of similar networks. We have extended the results in the study of heterogeneity in the RBNs and LSM beyond the in-degree heterogeneity and studied the dynamical effects of function heterogeneity and interconnect locality.

This work opens up the door to many future exploration. The real cause behind the effects of heterogeneity in network structure and functions need indepth study. Furthermore, we need to investigate if in real-world computations the heterogeneous networks outperform the homogeneous ones, as predicted by 


\begin{tabular}{|c|c|c|c|c|c|c|c|c|}
\cline { 2 - 10 } \multicolumn{1}{c|}{} & \multicolumn{2}{c|}{ LSM } & \multicolumn{2}{c|}{ Oscillators } & \multicolumn{2}{c|}{ RBN } & \multicolumn{2}{c|}{ SF RBN } \\
\cline { 2 - 10 } \multicolumn{1}{c|}{} & het. & hom. & het. & hom. & het. & hom. & het. & hom. \\
\hline in-degrees & poor & rich & poor & rich & rich & poor & rich & poor \\
\hline functions & no effect & no effect & n/a & n/a & rich & unpredictable & n/a & n/a \\
\hline interconnect & poor & rich & n/a & n/a & poor & rich & n/a & n/a \\
\hline
\end{tabular}

Table 6.1: This table summarizes the effects of heterogeneity in different aspects of RBN and LSM on the richness of dynamics. We compared our LSM results to the result for coupled oscillator networks [64]. We also compared our RBN results to scale-free RBNs $[5,96]$. Our result in the dynamical richness of the network for heterogeneous and homogeneous networks agree with the existing studies on similar networks.

the richer dynamics discovered in this study.

In the future, we may extend this work by rigorously studying the predictive power of the entropy measure as an indicator of heterogeneity, over systems dynamics and network properties. We might be able to use the rich literature of information theory to determine the information transfer between the entropy of the network components and the network dynamics. Furthermore, we can bridge the gap between pure theoretical analysis and study the computational power by training the RBN and LSM reservoirs to solve task and find if the results of our study can correlate with the actual task solving capability of RBN and LSM. 


\section{REFERENCES}

[1] Abelson, H., Allen, D., Coore, D., Hanson, C., Homsy, G., Knight, J. T. F., Nagpal, R., Rauch, E., Sussman, G. J., And Weiss, R. Amorphous computing. Commun. ACM 43, 5 (May 2000), 7482.

[2] Adar, R., Benenson, Y., Linshiz, G., Rosner, A., Tishby, N., And Shapiro, E. Stochastic computing with biomolecular automata. Proceedings of the National Academy of Sciences of the United States of America 101, 27 (2004), 9960-9965.

[3] Albert, R. Scale-free networks in cell biology. Arxiv preprint q-bio (Jan 2005).

[4] Albert, R., And Barabási, A. Statistical mechanics of complex networks. Rev. Mod. Phys. 74, 1 (2002), 47-97.

[5] Aldana, M. Boolean dynamics of networks with scale-free topology. Physica D: Nonlinear Phenomena (Jan 2003).

[6] Aldana, M., Coppersmith, S., and Kadanoff, L. P. Boolean dynamics with random couplings. In Perspectives and Problems in Nonlinear Science, E. Kaplan, J. E. Marsden, and K. R. Sreenivasan, Eds., vol. 93. Springer, May 2002, pp. 23-90.

[7] Amaral, L. A. N., Scala, A., BarthélÉmy, M., and Stanley, H. E. Classes of small-world networks. Proceedings of the National Academy of Sciences 97, 21 (10 2000), 11149-11152. 
[8] Amarnath, A., Damera, P., Goudarzi, A., And Teuscher, C. Latency and power consumption in unstructured nanoscale Boolean networks. In Proceedings of the $11^{\text {th }}$ International Conference on Nanotechnology (IEEE Nano 2011) (Cincinnati, OH, USA, 2011), IEEE, pp. 854-859.

[9] Arenas, A., Fernández, A., And Gómez, S. Analysis of the structure of complex networks at different resolution levels. New Journal of Physics 10 (May 2008), 3039.

[10] Ashby, W. R. Design for a Brain. John Wiley \& Sons. Inc., New York, NY, Jan 1960.

[11] Bar-Ziv, R. Dna circuits get up to speed. Science 318, 5853 (2007), 10781079.

[12] BarabÁsi, A., And Albert, R. Emergence of scaling in random networks. Science 286, 5439 (1999), 509.

[13] Barabasi, A.-L. The network takeover. Nat Phys 8, 1 (01 2012), 14-16.

[14] Bastolla, U., And Parisi, G. Relevant elements, magnetization and dynamical properties in Kauffman networks: A numerical study. Physica D: Nonlinear Phenomena 115, 3-4 (1998), 203-218.

[15] BegGs, J. M. The criticality hypothesis: how local cortical networks might optimize information processing. Philosophical Transactions of the Royal Society A: Mathematical, Physical and Engineering Sciences 366, 1864 (2008), $329-343$.

[16] Benenson, Y., Adar, R., Paz-Elizur, T., Livneh, Z., And Shapiro, E. DNA molecule provides a computing machine with both data and fuel. Proceedings of the National Academy of Sciences 100, 5 (2003), 2191-2196. 
[17] Bennett, H. S. Will future measurement needs of the semiconductor industry be met? Journal of Research of the National Institutes of Standards and Technologies 112 (2007), 25-38.

[18] Bertschinger, N., And Natschläger, T. Real-time computation at the edge of chaos in recurrent neural networks. Neural computation 16, 7 (2004), 1413-1436.

[19] Bilke, S., And Suunnesson, F. Stability of the Kauffman model. Physical Review E 65, 1 (2001), 16129.

[20] Boccaletti, S., Latora, V., Moreno, Y., Chavez, M., And Hwang, D. Complex networks: Structure and dynamics. Physics reports 424, 4-5 (2006), 175-308.

[21] Boedecker, J., Obst, O., Mayer, N. M., and Asada, M. Initialization and self-organized optimization of recurrent neural network connectivity. HFSP Journal 3, 5 (2009), 340-349.

[22] Bornholdt, S., AND RÖHL, T. Self-organized critical neural networks. Physical Review E 67 (2003), 066118.

[23] Bradde, S., Caccioli, F., Dall'asta, L., and Bianconi, G. Critical fluctuations in spatial complex networks. Phys. Rev. Lett. 104, 21 (May 2010), 218701.

[24] Büsing, L., Schraumen, B., And Legenstein, R. Connectivity, dynamics, and memory in reservoir computing with binary and analog neurons. Neural Computation 22, 5 (2010), 1272-1311.

[25] Caccioli, F., And Dall'Asta, L. Non-equilibrium mean-field theories on scale-free networks. Journal of Statistical Mechanics: Theory and Experiment 2009 (2009), P10004. 
[26] Chen, J., And Wood, D. H. Computation with biomolecules. Proceedings of the National Academy of Sciences 97, 4 (2000), 1328-1330.

[27] Darabos, C., Tomassini, M., and Giacobini, M. Dynamics of unperturbed and noisy generalized boolean networks. Journal of theoretical biology 260, 4 (2009), 531-544.

[28] De Arcangelis, L., And Herrmann, H. J. Learning as a phenomenon occurring in a critical state. Proceedings of the National Academy of Sciences 107, 9 (Mar 2010), 3977-81.

[29] De Arcangelis de Arcangelis, L., Perrone-Capano, C., And HerRMAnn, H. J. Self-organized criticality model for brain plasticity. Phys. Rev. Lett. 96 (Jan 2006), 028107.

[30] Derrida, B., And Pomeau, Y. Random networks of automata: A simple annealed approximation. EPL (Europhysics Letters) 1 (1986), 45.

[31] Dorogovtsev, S., Goltsev, A., and Mendes, J. Critical phenomena in complex networks. Reviews of Modern Physics 80, 4 (2008), 1275-1335.

[32] Dorogovtsev, S. N., And Mendes, J. F. F. Evolution of Networks: From Biological Nets to the Internet and $W W W$. Oxford University Press, New York, NY, 2003.

[33] Erdös, P., And RÉnyi, A. On random graphs. Publ. Math. Debrecen 6 (1959), 290-297.

[34] Fernando, C., And Sojakka, S. Pattern recognition in a bucket. In Proceedings of the 7th European Conference on Advances in Artificial Life (ECAL 2003) (2003), vol. 2801 of LNCS, Springer, pp. 588-597. 
[35] Flyvbjerg, H. An order parameter for networks of automata. J. Phys. A 21, 19 (1988), L955.

[36] Gershenson, C. Classification of random boolean networks. pp. 1-8.

[37] Gershenson, C. Introduction to random boolean networks. Arxiv preprint nlin (Jan 2004).

[38] Gómez, S., Arenas, A., Borge-Holthoefer, J., Meloni, S., And Moreno, Y. Discrete-time markov chain approach to contact-based disease spreading in complex networks. EPL (Europhysics Letters) 89, 3 (2010), 38009.

[39] Goudarzi, A., Teuscher, C., And Gulbahce, N. Learning and generalization in random automata networks. In Bionetics 2010: The 5th International ICST Conference on Bio-Inspired Models of Network, Information, and Computing Systems (Boston, MA, Dec 1-3 2010).

[40] Goudarzi, A., Teuscher, C., Gulbahce, N., and Rohlf, T. Emergent criticality through adaptive information processing in boolean networks. Phys. Rev. Lett. 108 (2012), 128702.

[41] Guimerà, R., And Amaral, L. A. N. Cartography of complex networks: modules and universal roles. J Stat Mech 2005, P02001 (2005), nihpa35573.

[42] Haselman, M., And Hauck, S. The future of integrated circuits: A survey of nanoelectronics. Proceedings of the IEEE 98, 1 (2010), 11-38.

[43] Hogg, R., McKean, J., And Craig, A. T. Introduction to Mathematical Statistics (7th Edition). Prentice Hall, New Jersey, USA, 2012.

[44] Hong, H., HA, M., And PARK, H. Finite-size scaling in complex networks. Phys. Rev. Lett. 98, 25 (2007), 258701. 
[45] JAEGER, H. The "echo state" approach to analysing and training recurrent neural networks. Tech. Rep. GMD Rep. 148, St. Augustin: German National Research Center for Information Technology, 2001.

[46] JaEger, H. Short term memory in echo state networks. Tech. Rep. GMD Report 152, GMD-Forschungszentrum Informationstechnik, 2002.

[47] JAEGER, H. Tutorial on training recurrent neural networks, covering BPPT, RTRL, EKF and the "echo state network" approach. 2002.

[48] JAEger, H. Adaptive nonlinear system identification with echo state networks.

[49] KARI, J. Theory of cellular automata: a survey. Theoretical Computer Science 334, 1-3 (2005).

[50] Kauffman, S. Emergent properties in random complex automata. Physica D 10, 1-2 (1984), 145-156.

[51] Kauffman, S., Peterson, C., Samuelsson, B., and Troein, C. Genetic networks with canalyzing boolean rules are always stable. Proc Natl Acad Sci U S A 101, 49 (2004), 17102-7.

[52] Kauffman, S. A. Metabolic stability and epigenesis in randomly constructed genetic nets. Journal of theoretical biology 22, 3 (1969), 437-467.

[53] Kauffman, S. A. Requirements for evolvability in complex systems: orderly dynamics and frozen components. Physica D 42, 1-3 (1990), 135-152.

[54] Kauffman, S. A. Antichaos and adaptation. Scientific American 265, 2 (1991), 78-84. 
[55] Kauffman, S. A. Coevolution to the edge of chaos: Coupled fitness landscapes, poised states, and coevolutionary avalanches. Journal of Theoretical Biology 149, 4 (1991), 467-505.

[56] Kauffman, S. A. The Origins of Order: Self-Organization and Selection in Evolution. Oxford University Press, New York, NY, 1993.

[57] Kauffman, S. A., And Weinberger, E. D. The NK model of rugged fitness landscapes and its application to maturation of the immune response. Journal of Theoretical Biology 141, 2 (1989), 211-45.

[58] Kaufman, V., And Drossel, B. Relevant components in critical random boolean networks. New Journal of Physics 8, 10 (2006), 228.

[59] Kim, H., Toroczkai, Z., Erdős, P. L., Miklós, I., And SzÉkely, L. A. Degree-based graph construction. Journal of Physics A: Mathematical and Theoretical 42, 39 (2009), 392001.

[60] Kinouchi, O., And Copelli, M. Optimal dynamical range of excitable networks at criticality. Nat Phys 2, 5 (05 2006), 348-351.

[61] Kirkpatrick, S., Gelatt, C. D., and Vecchi, M. P. Optimization by simulated annealing. Science 220, 4598 (1983), 671-680.

[62] Kleinberg, J. M. Navigation in a small world. Nature 406, 6798 (2000), $845-845$.

[63] Langton, C. Computation at the edge of chaos: Phase transitions and emergent computation. Physica D (1990).

[64] Larremore, D. B., Shew, W. L., and Restrepo, J. G. Predicting criticality and dynamic range in complex networks: Effects of topology. Phys. Rev. Lett. 106, 5 (2011), 058101. 
[65] Lee, S. H., Ha, M., Jeong, H., Noh, J. D., And Park, H. Critical behavior of the ising model in annealed scale-free networks. Physical Review E 80, 5 (2009), 051127.

[66] Lendaris, G. G. On systemness and the problem solver: Tutorial comments. IEEE Transactions on Systems, Man and Cybernetics 16, 4 (1986), 603-610.

[67] Li, W., AND PACKARd, N. The structure of the elementary cellular automata rule space. Complex systems 4, 3 (1990), 281-297.

[68] Li, W., Packard, N., And Langton, C. Transition phenomena in cellular automata rule space. Physica D 45, 1-3 (1990), 77-94.

[69] LiU, M., AND BAssler, K. Finite size effects and symmetry breaking in the evolution of networks of competing boolean nodes. J. Phys. A 44 (2011), 045101.

[70] LiU, M., And BAssler, K. E. Emergent criticality from coevolution in random boolean networks. Physical Review E 74, 4 (2006), 41910.

[71] Lizier, J., Prokopenko, M., And Zomaya, A. The information dynamics of phase transitions in random boolean networks. In Eleventh International Conference on the Simulation and Synthesis of Living Systems (ALife XI) (Cambridge, MA, USA, August 2008), MIT Press, pp. 374-381.

[72] Lizier, J. T., Prokopenko, M., and Zomaya, A. Y. Coherent information structure in complex computation. Theory in Biosciences To appear (2010).

[73] Lu, Q., And Teuscher, C. Damage spreading in spatial and small-world random boolean networks. arXiv:0904.4052v1 [cond-mat.dis-nn] (2009). 
[74] Lukosevicius, M., And Jaeger, H. Reservoir computing approaches to recurrent neural network training. Computer Science Review 3, 3 (2009), $127-149$.

[75] Maass, W., NatschläGer, T., And Markram, H. Real-time computing without stable states: a new framework for neural computation based on perturbations. Neural computation 14, 11 (2002), 2531-60.

[76] Mesot, B., And Teuscher, C. Deducing local rules for solving global tasks with random boolean networks. Physica D 211, 1-2 (2005), 88-106.

[77] Mitchell, M., Crutchfield, J., And Hraber, P. Evolving cellular automata to perform computations: Mechanisms and impediments. Physica D 75 (1994), 361-391.

[78] Mitchell, M., Crutchfield, J. P., And Hraber, P. T. Dynamics, computation, and the "edge of chaos": A re-examination. In Complexity: Metaphors, Models, and Reality. Reading, G. Cowan, D. Pines, and D. Melzner, Eds. Addison-Wesley, Reading, MA, 1994, pp. 497-513.

[79] Mitchell, M., Hraber, P. T., And Crutchfield, J. P. Revisiting the edge of chaos: Evolving cellular automata to perform computations. Complex systems 7 (1993), 89-130.

[80] Moreira, A. A., And Amaral, L. A. N. Canalizing kauffman networks: Nonergodicity and its effect on their critical behavior. Phys. Rev. Lett. 94, $21(2005), 218702$.

[81] Natschlaeger, T., And MaAss, W. Information dynamics and emergent computation in recurrent circuits of spiking neurons. In Proc. of NIPS 2003, Advances in Neural Information Processing Systems (Cambridge, 2004), 
S. Thrun, L. Saul, and B. Schoelkpf, Eds., vol. 16, MIT Press, pp. 12551262.

[82] Newman, M. The structure and function of complex networks. SIAM Review 45, 2 (2003), 167-256.

[83] Newman, M. Fast algorithm for detecting community structure in networks. Physical Review E 69, 6 (2004), 066133.

[84] Newman, M. E. J. Mixing patterns in networks. Phys. Rev. E 67 (2003), 026126.

[85] Newman, M. E. J., Strogatz, S. H., And Watts, D. J. Random graphs with arbitrary degree distributions and their applications. Phys. Rev. E 64 (2001), 026118.

[86] Oltean, M. Unconventional computing: a short introduction. Stud. Univ. Babeş-Bolyai, Inform. 54 (2007), 85-96.

[87] Petermann, T., And De Los Rios, P. Physical realizability of smallworld networks. Phys. Rev. E 73 (2006), 026114.

[88] Reichl, M. D., And Bassler, K. E. Canalization in the critical states of highly connected networks of competing boolean nodes. Phys. Rev. E 84 (2011), 056103.

[89] Ribeiro, A. S., Kauffman, S. A., Lloyd-Price, J., Samuelsson, B., AND Socolar, J. E. S. Mutual information in random boolean models of regulatory networks. Phys. Rev. E 77, 1 (2008), 011901.

[90] Rohlf, T. Self-organization of heterogeneous topology and symmetry breaking in networks with adaptive thresholds and rewiring. EPL (Europhysics Letters) 84 (2008), 10004. 
[91] Rohlf, T., And Bornholdt, S. Criticality in random threshold networks: annealed approximation and beyond. Physica A: Statistical Mechanics and its Applications 310, 1-2 (2002), 245-259.

[92] Rohlf, T., Gulbahce, N., And Teuscher, C. Damage spreading and criticality in finite random dynamical networks. Phys. Rev. Lett. 99, 24 (2007), 248701.

[93] Rosca, J. P. Entropy-driven adaptive representation. In Proceedings of the Workshop on Genetic Programming: From Theory to Real-World Applications (Tahoe City, California, USA, 9 July 1995), J. P. Rosca, Ed., pp. 23-32.

[94] Rubinov, M., And Sporns, O. Complex network measures of brain connectivity: uses and interpretations. Neuroimage 52, 3 (2010), 1059-69.

[95] Schraumen, B., Verstraeten, D., and Campenhout, J. V. An overview of reservoir computing: theory, applications and implementations. In Proceedings of the 15th European Symposium on Artificial Neural Networks (2007), pp. 471-482.

[96] Serra, R., Villani, M., and Agnostini, L. On the dynamics of scalefree boolean networks. Lecture Notes in Computer Science 2859 (2003), 43-49.

[97] Serra, R., Villani, M., And Agostini, L. On the dynamics of random boolean networks with scale-free outgoing connections. Physica A 339, 3-4 (2004), 665-673.

[98] Service, R. F. Dna nanotechnology grows up. Science 332, 6034 (2011), $1140-1143$.

[99] Shapiro, E., AND GiL, B. Rna computing in a living cell. Science 322, 5900 (2008), 387-388. 
[100] Shmulevich, I., And Kauffman, S. Activities and sensitivities in boolean network models. Phys. Rev. Lett. 93, 4 (2004), 048701.

[101] SieradzKI, K. Potential solutions for creating responsive materials. Science 332, 6034 (2011), 1158-1159.

[102] Snyder, D., Goudarzi, A., And Teuscher, C. Finding optimal random boolean networks for reservoir computing. In Alife XIII (in review) (2012).

[103] Socolar, J. E. S., And Kauffman, S. A. Scaling in ordered and critical random boolean networks. Phys. Rev. Lett. 90, 6 (2003), 068702.

[104] Sole, R., Luque, B., And Kauffman, S. Phase transition in random networks with multiple states. Arxiv preprint adap-org/9907011 (1999).

[105] Sole, R. V., And VAlverde, S. Information theory of complex networks: on evolution and architectural constraints. Lect. Notes Phys. 650, 189-207 (2004).

[106] Sporns, O. Networks of the Brain. The MIT Press, Cambridge, MA, 2011.

[107] Teuscher, C. Turing's Connectionism. An Investigation of Neural Network Architectures. Springer-Verlag, London, 2002.

[108] Teuscher, C., Gulbahce, N., Rohlf, T., And Goudarzi, A. Random dynamical network automata for nanoelectronics: A robustness and learning perspective. In Theoretical and Technological Advancements in Nanotechnology and Molecular Computation: Interdisciplinary Gains, B. MacLennan, Ed. IGI Global, Hershey, NY, 2011, ch. 19, pp. 295-314.

[109] Tomassini, M., Giacobini, M., And Darabos, C. Evolution of smallworld networks of automata for computation. Lecture Notes in Computer Science 3242 (2004), 672-681. 
[110] Tomassini, M., Giacobini, M., And Darabos, C. Evolution and dynamics of small-world cellular automata. Complex systems (2005).

[111] Von Neumann, J. Theory of Self-Reproducing Automata. University of Illinois Press, Urbana, Illinois, 1966.

[112] Wang, D., And Gribskov, M. Examining the architecture of cellular computing through a comparative study with a computer. Journal of The Royal Society Interface 2, 3 (2005), 187-195.

[113] Watts, D., And Strogatz, S. Collective dynamics of 'smallworld'networks. Nature 393, 6684 (1998), 440-442.

[114] Wolfram, S. Statistical mechanics of cellular automata. Rev. Mod. Phys. 55, 3 (1983), 601-644.

[115] Wootters, W., And Langton, C. Is there a sharp phase transition for deterministic cellular automata? Physica D 45, 1-3 (1990).

[116] Xie, Z., Liu, S. J., Bleris, L., And Benenson, Y. Logic integration of mrna signals by an rnai-based molecular computer. Nucleic Acids Research 38, 8 (2010), 2692-2701. 